\title{
Systemic and renal hemodynamic changes with the menstrual cycle : a study in normal women and women with a history of preeclampsia
}

Citation for published version (APA):

van Beek, E. (1999). Systemic and renal hemodynamic changes with the menstrual cycle : a study in normal women and women with a history of preeclampsia. [Doctoral Thesis, Maastricht University]. Universiteit Maastricht. https://doi.org/10.26481/dis.19990226eb

Document status and date:

Published: 01/01/1999

DOI:

10.26481/dis.19990226eb

Document Version:

Publisher's PDF, also known as Version of record

Please check the document version of this publication:

- A submitted manuscript is the version of the article upon submission and before peer-review. There can be important differences between the submitted version and the official published version of record.

People interested in the research are advised to contact the author for the final version of the publication, or visit the DOI to the publisher's website.

- The final author version and the galley proof are versions of the publication after peer review.

- The final published version features the final layout of the paper including the volume, issue and page numbers.

Link to publication

\footnotetext{
General rights rights.

- You may freely distribute the URL identifying the publication in the public portal. please follow below link for the End User Agreement:

www.umlib.nl/taverne-license

Take down policy

If you believe that this document breaches copyright please contact us at:

repository@maastrichtuniversity.nl

providing details and we will investigate your claim.
}

Copyright and moral rights for the publications made accessible in the public portal are retained by the authors and/or other copyright owners and it is a condition of accessing publications that users recognise and abide by the legal requirements associated with these

- Users may download and print one copy of any publication from the public portal for the purpose of private study or research.

- You may not further distribute the material or use it for any profit-making activity or commercial gain

If the publication is distributed under the terms of Article $25 \mathrm{fa}$ of the Dutch Copyright Act, indicated by the "Taverne" license above, 
Systemic and renal hemodynamic changes with the menstrual cycle 
C E. van Beek, Maastricht 1999

ISBN 9090123725

Vormgeving en druk: Datawyse I Universitaire Pers Maastricht 


\section{Systemic and renal hemodynamic changes with the menstrual cycle}

A study in normal women and women with a history of preeclampsia

\section{Proefschrift}

ter verkrijging van de graad van doctor

aan de Universiteit Maastricht,

op gezag van de Rector Magnificus,

Prof. dr. A.C. Nieuwenhuijzen Kruseman,

volgens het besluit van het College van Decanen,

in het openbaar te verdedigen

op vrijdag 26 februari 1999 om 16.00 uur

door

Erik van Beek 
Promotores

Prof. dr. J de Haan

Prof. dr. P.W. de Leeuw

Co-promotor

dr. L.L.H. Peeters

Beoordelingscommissie

Prof. dr. H.A.J. Struijker Boudier (voorzitter)

Prof. dr. J.L.H. Evers

Prof. dr. E.M. Symonds (University of Nottingham, England)

Prof. dr. Th. Thien (Katholieke Universiteit Nijmegen)

Prof. dr. H.J.J. Wellens

The studies described in this thesis and the publication of the thesis were financially supported by the Dutch Kidney Foundation (project C 94.1372) 
voor Margreet 


\section{Contents}

Abbreviations $\quad 8$

CHAPTER 1

Introduction 11

\section{CHAPTER 2}

The pathogenesis of preeclampsia: a comprehensive model

\section{CHAPTER 3}

Peripheral hemodynamic and renal function in relation to the menstrual cycle $\quad 23$

\section{CHAPTER 4}

Body fluid volume changes during the menstrual cycle

\section{CHAPTER 5}

Vascular resistance in relation to the menstrual cycle $\quad 47$

\section{CHAPTER 6}

The relative role of progesterone and $17 \beta$-estradiol in

hemodynamic changes during the menstrual cycle

\section{CHAPTER 7}

Persistent abnormalities in plasma volume and renal hemodynamics in patients with a history of preeclampsia

CHAPTER 8

Changes in hemodynamics and fluid volume with the menstrual cycle in women with a history of preeclampsia

\section{CHAPTER 9}

General discussion $\quad 101$

CHAPTER 10

References $\quad 107$ 
CHAPTER 11

Summary 119

Samenvatting $\quad 122$

PUBLICATIONS 125

NAWOORD $\quad 126$

CURRICULUM VITAE 127 


\section{Abbreviations}

\begin{tabular}{|c|c|}
\hline ACA & anticardiolipin antibodies \\
\hline Aldo & aldosterone \\
\hline AngII & angiotensin II \\
\hline ANP & atrial natriuretic peptide \\
\hline APRC & active plasma renin concentration \\
\hline APS & antiphospholipid syndrome \\
\hline BMI & body mass index \\
\hline BP & blood pressure \\
\hline BV & blood volume \\
\hline CI & cardiac index \\
\hline $\mathrm{CO}$ & cardiac output \\
\hline $\mathrm{CV}$ & coefficient of variation \\
\hline DBP & diastolic blood pressure \\
\hline ECFV & extracellular fluid volume \\
\hline ERBF & effective renal blood flow \\
\hline ERPF & effective renal plasma flow \\
\hline FBF & forearm blood flow \\
\hline FF & filtration fraction \\
\hline FP & follicular phase \\
\hline FVR & forearm vascular resistance \\
\hline GA & gestational age \\
\hline GFR & glomerular filtration fraction \\
\hline HELLP & hemolysis, elevated liver enzymes, low platelets \\
\hline $\mathrm{HHC}$ & hyperhomocysteinemia \\
\hline HR & heart rate \\
\hline $\mathrm{Ht}$ & hematocrit \\
\hline HT & chronic hypertension \\
\hline IUGR & intra-uterine growth retardation \\
\hline LA & lupus anticoagulant \\
\hline LP & luteal phase \\
\hline LVW & left ventricular work \\
\hline MAP & mean arterial pressure \\
\hline PAH & para-aminohippurate sodium \\
\hline PE & preeclampsia \\
\hline
\end{tabular}


PV plasma volume

RAA renin-angiotensin-aldosterone

RBF renal blood flow

RF renal fraction

RVR renal vascular resistance

SBP systolic blood pressure

SD standard deviation

SV stroke volume

TP thrombophilic disorder

TPR total peripheral vascular resistance

TT transit time 



\section{$1{ }^{\text {CHAMTrER }}$ \\ 1 General introduction}

Elevated blood pressure during pregnancy can be subdivided into a form that antedates pregnancy (chronic hypertension), a form that is confined to the period of pregnancy and also complicated by proteinuria (preeclampsia), and a form in which preeclampsia is superimposed on chronic hypertension. In preeclampsia the vascular pathology is characterized by the combination of a generalized vasospasm, enhanced thrombocyte aggregation and increased capillary permeability. These symptoms are consistent with endothelial dysfunction and may interfere with organ perfusion.

High blood pressure complicates almost $10 \%$ of all pregnancies ${ }^{1}$. It is, together with obstetric hemorrhage, embolism and obstetric infection, the most common cause of maternal mortality. In a period of four years (1987-1990), it was directly responsible for 256 deaths on a total of 1453 maternal deaths in the United States, corresponding with 1.6 maternal deaths per 100,000 live births ${ }^{2}$. The working group on maternal mortality of the Dutch Society of Obstetrics and Gynecology reported 13 cases on a total of 66 maternal deaths in the Netherlands between 1988 and 1992, corresponding with 1.7 maternal deaths per 100,000 live births ${ }^{3}$. Maternal hypertension is also an important cause of perinatal morbidity and mortality, as it predisposes for intra-uterine growth retardation and iatrogenic preterm delivery performed on fetal or maternal indication.

Since the cause of preeclampsia is unknown, current treatment modalities are limited to symptomatic relief and optimal timing of delivery. Unfortunately, only the latter, rather aggressive treatment option can be seen as definitive, for it removes the diseased tissue: the placenta. A more causal management and treatment of preeclampsia will not be achieved until the pathogenesis of the disorder is fully disclosed ${ }^{4}$.

In their studies Duvekot et al. 5,6 provided evidence for a defective maternal hemodynamic adaptation in the first weeks of pregnancy preceding defective fetal growth later on in pregnancy. Their observations in the latter group of women suggested volume maladaptation in the first eight weeks of pregnancy in this particular group. It is conceivable that this seeming persistence of a state of vascular underfill in early pregnancy triggers or at least contributes to the development of intra-uterine growth retardation (IUGR) and preeclampsia in advanced pregnancy. The relative hypovolemia may lead to a raise in both sympathetic tone and RAA-system activity in the post-implantation period, causing a redistribution of the cardiac output away from the invading trophoblast. The latter can be expected 
to interfere with placentation. Poor placentation is generally considered to play a central role in the pathogenesis of preeclampsia.

Central and renal hemodynamic changes in the menstrual cycle resemble those in early pregnancy. Firstly, the mean arterial pressure in the luteal phase (LP) has decreased relative to the follicular phase (FP) 7 . This decrease is accompanied by a fall in osmotic thresholds for thirst and vasopressin release ${ }^{8}$, suggesting that these phenomena may represent compensations in response to a primary decrease in vascular tone. Secondly, most reports on this topic provide experimental evidence for a luteal rise in glomerular filtration rate (GFR) ${ }^{9-12}$. This luteal rise in GFR is probably flow-induced as there is some evidence for a concomitant increase in effective renal plasma flow (ERPF) ${ }^{11}$. However, the use of suboptimal markers for GFR and ERPF together with a lack of standardization of sodium intake throughout the menstrual cycle do not allow firm conclusions on this matter.

Thirdly and lastly, an increased activity of the RAA-system during the LP as compared to the FP has been demonstrated ${ }^{13-15}$.

The combination of the above mentioned hemodynamic maladaptation in early pregnancy in case of IUGR and the resemblance of the hemodynamic changes in the LP, relative to the FP, with those in early normal pregnancy led us to the following hypothesis:

In women with preeclamptic pregnancy, maladaptation of central and renal hemodynamics in early pregnancy is mirrored by abnormal hemodynamic changes during the menstrual cycle.

To test this hypothesis, we studied central and renal hemodynamic changes during the menstrual cycle in healthy subjects using the hemodynamic adaptations as observed in early pregnancy as a reference. In an initial pilot-study (Chapter 3) we addressed the question whether there were indeed indirect signs for a generalized vascular relaxation in the LP. Afterwards, we focused on changes in overall peripheral, renal, and forearm vascular resistances during ovulatory (Chapter 5) and anovulatory (Chapter 6) menstrual cycles, in subjects on a standardized salt-intake. During the measurement sessions we also studied possible changes in body fluid volume (Chapter 4 and 6).

In addition, we investigated whether renal hemodynamics and plasma volume in the LP of the menstrual cycle differ between parous subjects with or without preeclampsia in their recent history (Chapter 7). Finally, we compared cyclic changes in renal hemodynamics and plasma volume in these two categories of subjects to determine whether ex-preeclamptics elicit an abnormal hemodynamic cyclicity during the menstrual cycle (Chapter 8). 


\section{Pathogenesis of preeclampsia: a comprehensive model}

E. van Beek, MD, L.L.H. Peeters, MD, PhD

Departments of Obstetrics and Gynecology,

University Hospital Maastricht, Maastricht, The Netherlands

Obstet Gynecol Surv 1998; 53:233-239 
The objective of this review was to provide a comprehensive and practical concept on the pathogenesis of preeclampsia on the basis of the currently available scientific evidence.

A MEDLINE search was performed of English language articles published between 1966 and 1997, supplemented with references cited in relevant research articles.

Using our data sources we developed a scheme describing the sequence of events between implantation and the time of manifest clinical disease characterized by generalized endothelial cell dysfunction. A yet unidentified toxic circulating factor released by the ischemic placenta, is held responsible for the impaired endothelial cell function. Particularly, epidemiological studies point to a concept in which immune maladaptation to the fetal allograft plays a key role in causing defective placentation leading to placental ischemia. The incidence of preeclampsia in sisters and daughters of women who have had preeclampsia is raised. Disease states with vascular involvement, like chronic hypertension and diabetes mellites, are associated with an increased risk for preeclampsia. Recently subclinical abnormalities in hemostasis, metabolism and volume homeostasis have been described in patients with a history of preeclampsia.

Placental ischemia secondary to defective placentation, a prerequisite for the development of preeclampsia, has a multifactorial origin consisting of three major components: immune maladaptation, genetic predisposition and vascular mediated factors. Probably, a summation of these factors will determine whether a pregnant woman is to develop the syndrome. The recently described subclinical abnormalities in hemostasis, metabolism and vascular function in patients with a history of preeclampsia might give the clinician the opportunity to reduce the recurrence risk by pharmacotherapeutic intervention.

High blood pressure complicates almost 10 percent of all pregnancies ${ }^{1}$. It is, together with obstetric hemorrhage, embolism and obstetric infection, the most common cause of maternal mortality. In a period of 4 years (1987-1990), it was directly responsible for 256 deaths of a total of 1,453 maternal deaths in the United States, corresponding with 1.6 maternal deaths per 100,000 live births ${ }^{2}$. The working group on maternal mortality of the Dutch Society of Obstetrics and Gynecology reported 13 cases of a total of 66 maternal deaths in the Netherlands between 1988 and 1992, corresponding with 1.7 maternal deaths per 100,000 live births ${ }^{3}$.Maternal hypertension is also an important cause of perinatal morbidity and mortality, because it predisposes for intrauterine growth retardation and iatrogenic preterm delivery performed for fetal or maternal indications. Classifications of hypertensive disorders of pregnancy have been made by the World Health 
Table 1. Classification of hypertensive disorders of pregnancy.

Chronic hypertension

Preeclampsia/eclampsia

Preeclampsia superimposed

on chronic hypertension

Transient hypertension
Known hypertension before pregnancy or rise in blood pressure to $\geq 140 / 90 \mathrm{~mm} \mathrm{Hg}$ before 20 weeks amenorrhea. Increased blood pressure accompanied by proteinuria ( $>300$ $\mathrm{mg} / 24$ hour specimen), edema or both, occurring almost exclusively after 20 weeks amenorrhea. Rise in blood pressure by $\geq 15 \mathrm{~mm} \mathrm{Hg}$ diastolic, by $\geq 30 \mathrm{~mm} \mathrm{Hg}$ systolic from measurement in early pregnancy or to $\geq 140 / 90$ in late pregnancy if prior blood pressure is not known. Eclampsia is the occurrence of seizures in a patient with preeclampsia.

The combination of chronic hypertension and preeclampsia. Rise in blood pressure as for preeclampsia without proteinuria.

Organization (WHO), American College of Obstetrics and Gynecology (ACOG), the International Society for the Study of Hypertension (ISSH) and the U.S. National Institutes of Health Working Group on Hypertension in Pregnancy ${ }^{1}$. A description of the latter classification, which is widely accepted for its clarity and simplicity, is outlined in Table 1 . The most important consideration in the classification of elevated blood pressure during pregnancy is the subdivision of hypertension into a form that antedates pregnancy (chronic hypertension) and a form that is confined to the period of pregnancy (preeclampsia). In the latter condition the vascular pathology is characterized by the combination of a generalized vasospasm, enhanced thrombocyte aggregation and increased capillary permeability all of which have a negative impact on organ perfusion. The objective of this review is to provide a practical and comprehensive model about the pathogenesis of preeclampsia on the basis of currently available scientific evidence.

\section{Endothelial cell dysfunction, the final common pathway}

Many observations point to a central role of the endothelial cells in the pathogenesis of pre-eclampsia. First, there is morphologic evidence of endothelial cell injury in the kidney known as glomerular endotheliosis ${ }^{16}$. Shanklin et al. described ultrastructural endothelial injury in the placental bed and adjacent uterine vessels ${ }^{17}$. Second, functional derangements or biochemical evidence of damaged endothelial cells have been described in preeclamptics: high circulating concentrations of the von Willebrand factor ${ }^{18,19}$, endothelin ${ }^{20,21}$, and the cellular epitope of fibronectin ${ }^{22-24}$. Disturbances of the total plasminogen activator (tPA) and plasmi- 
nogen activator/inhibitor balance and the prostacyclin $\left(\mathrm{PGI}_{2}\right) /$ thromboxane $\left(\mathrm{TXA}_{2}\right)$ balance are in favor of an increased tendency of thrombocytes to aggregate and of vessels to constrict ${ }^{25,26}$. These morphologic and functional changes of the endothelial cells can be held directly responsible for triggering the clinical syndrome: arterial vasospasm, increased thrombocyte aggregation and increased capillary permeability leading to hypertension, proteinuria, edema and sometimes thrombocytopenia and hypoperfusion of organs.

\section{Placental ischemia, the starting point}

Based on many reports, the placenta, like the endothelium, plays a key role in the pathogenesis of preeclampsia. Preeclampsia is an exclusive disease of pregnancy that implies that it will resolve within 48 hours after delivery of the placenta. The occurrence of preeclampsia in molar pregnancies supports the central role of the placenta in the development of the syndrome ${ }^{27}$. Similarly, the occurrence of preeclampsia in abdominal pregnancies emphasizes the irrelevance of the decidua in the pathogenesis of the disease ${ }^{28,29}$.

The following inferences support the concept that reduced placental perfusion has to play a major role in pregnancies eventually complicated by preeclampsia. In a normal pregnancy trophoblastic invasion is associated with striking changes in the arteries supplying the intervillous space. The diameter of spiral arteries proximal to the trumpet-shape outlet increases four- to six fold relative to that in nonpregnant women (Fig. 1). The endothelium is replaced by trophoblast, and the internal elastic lamina is replaced by trophoblast and an amorphous matrix that consists predominantly of fibrin deposits. These vascular changes extend from the intervillous space up to the inner third of the myometrium ${ }^{30,31}$. By contrast, these changes develop defectively in preeclamptic women and are also limited to the decidual portion of the spiral arteries, with myometrial segments maintaining their smooth muscle layer ${ }^{32}$ (Fig. 1). These vessels have diameters that are only about 40 percent of those of the vessels of normal pregnancies ${ }^{33}$. Some spiral arteries in decidua and the myometrium are occluded by fibrinoid material and surrounded by foam cells. These features, are known as atherosis, and resemble those seen with allograft rejection ${ }^{34}$. One consequence of the abnormal invasion of the spiral arteries is a deficient uteroplacental circulation already at the end of the first trimester. The latter is supported by histopathologic findings of villi in preeclamptic patients that consistently show a pattern of abnormalities characteristic for villous growth in a low oxygen environment ${ }^{35}$. Chronic reduction of the aortic pressure in rhesus monkeys can produce a syndrome resembling preeclampsia. This observation supports the hypothesis of a reduced perfusion of the implantation site already at the time of the initial trophoblast invasion ${ }^{36}$.

The pathway culminating in placental ischemia holds the key to our understanding of the pathogenesis of preeclampsia. Research today focuses on both this so- 

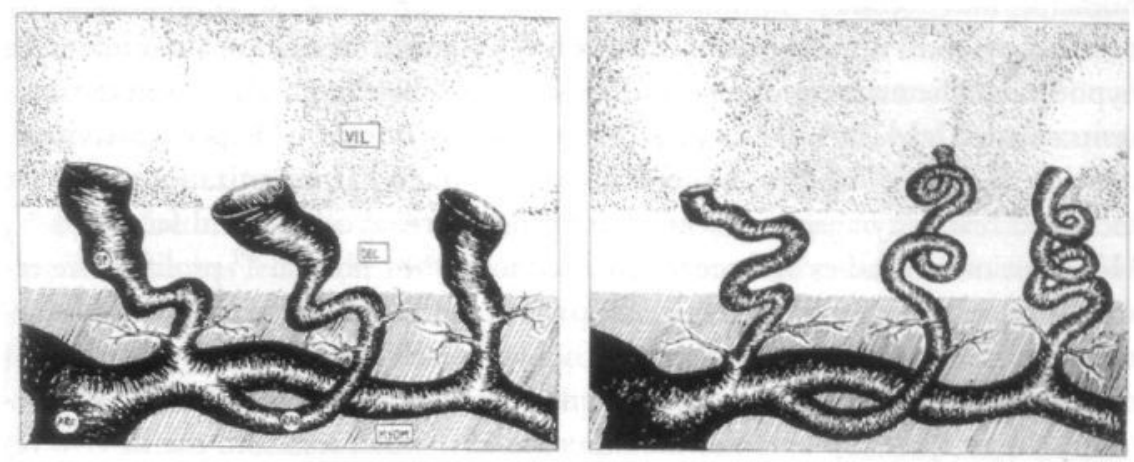

Figure 1. Diagram illustrating defective placentation. The left side of the diagram shows normal adaptation of spiral arteries to pregnancy, whereas the right side depicts this process in preeclamptics. Vil = intervillous space; $\mathrm{sp}=$ spiral artery; $\mathrm{dec}=$ decidua; rad = radial artery; $\operatorname{arc}=$ arcuate artery; myom = myometrium.

called "defective placentation" and the missing link between placental ischemia and generalized endothelial dysfunction. The available epidemiological evidence supports the view that the origin of this placentation defect is multifactorial, consisting of three major components: immune maladaptation, genetic predisposition and vascular-mediated factors. Probably, a summation of these factors will decide whether a pregnant woman is destined to develop the syndrome and its severity.

\section{Immune maladaptation}

The concept of an abnormal maternal immune response to the trophoblast is largely based on epidemiological studies. The risk of developing preeclampsia is reduced in subsequent pregnancies compared with the first provided conception is achieved by the same partner ${ }^{37,38}$. A spontaneous abortion or blood transfusion is associated with a lower risk of preeclampsia in a subsequent pregnancy 39,40 . Other studies associate preeclampsia with the frequency and duration of maternal exposure to semen ${ }^{41}$. There is an inverse correlation between the duration of cohabitation and the risk of developing preeclampsia ${ }^{42}$. Women using barrier methods of contraception have twice the likelihood of developing preeclampsia than women using oral contraceptives or an IUCD ${ }^{43}$. Finally, the incidence of preeclampsia in pregnancies from donor inseminations is higher than expected ${ }^{44}$. All these observations support an as yet undefined key role of the immune response of the mother to antigens on the trophoblast in the development of preeclampsia. It is tempting to speculate that the normal tolerance process of the fetal allograft develops abnormally. Many studies have been designed to compare 
humeral and cellular immune functions in pregnancies complicated by preeclampsia and normal pregnancies to find evidence for the impaired tolerance hypothesis. The majority of reports do not demonstrate modifications in the concentration of $\mathrm{IgM}, \mathrm{IgA}, \mathrm{IgG}$ and $\mathrm{IgD}$ levels during normal or hypertensive pregnancies ${ }^{45}$. Some studies showed abnormal maternal sensitization with an increased reactivity against placental antigens, paternal cells ${ }^{46}$ and fetal cells ${ }^{47}$. However, other studies demonstrated a reduced ${ }^{48}$ or normal ${ }^{49}$ proliferative response of maternal lymphocytes in the presence of paternal lymphocytes. Unfortunately, most of the results are in conflict with each other primarily, because of the presently limited understanding of normal fetal allograft tolerance. Furthermore, the relationship between the outcome of in-vitro tests and the in-vivo responses is still poorly defined ${ }^{45}$. Powerful protective mechanisms are likely to be activated to ensure the avoidance of graft rejection during pregnancy. The classical HLA class Ia antigens, expressed on all nucleated cells, normally serve as recognition structures for rejecting genetically foreign tissue by cytotoxic T-lymphocytes. Trophoblast cells are a notable exception. In these cells a class Ib HLA gene, HLA-G, is expressed ${ }^{50}$. The unique tissue distribution of HLA-G suggests that this molecule plays a key role in the induction of maternal tolerance in pregnancy 51,52 . An alternative hypothesis suggests that maternal production of blocking antibodies plays an important role in the survival of the conceptus as an allograft ${ }^{53}$. Decreased formation of these protective blocking antibodies could lead to a maternal immune response directed against endovascular and interstitial trophoblast invasion. Placental ischemia and activation of polynuclear neutrophils seem to be the most severe consequence of this abnormal maternal immune response, which triggers the chain of events with ultimate endpoint systemic endothelial damage/dysfunction and associated symptomatology of preeclampsia.

\section{Genetic predisposition}

In a prospective study in 1961, Chesley and co-workers were the first to describe the familial factor of preeclampsia ${ }^{54}$. They reported on a markedly increased incidence of preeclampsia and eclampsia in the first pregnancies of sisters (37 percent) and daughters ( 26 percent), of 187 eclamptic women relative to the incidence in the general population (8 percent) ${ }^{54,55}$. Studies addressing the mode of transmission are hampered by the slow reproductive cycle in the human and the confinement of the phenotype to pregnancy. Several hypotheses concerning the inheritance of a gene that regulates the susceptibility to preeclampsia have been proposed, all of them attempting to reconcile epidemiological data with a model of transmission. Sutherland et al. ${ }^{56}$ and Chesley et al. ${ }^{57}$ proposed a simple maternal recessive transmission with an estimated gene frequency of 0.237 (Scottish population) and 0.253 (New Jersey population), respectively. Both studies ruled out a significant genetic contribution by the fetus because they found a population 
incidence of preeclampsia for in-laws. For an Icelandic population, Arngrimsson et al. ${ }^{58}$ described two mathematical models with an equal fit: a recessive mode of transmission with a causal gene present in 31 percent of women and an autosomal dominant mode of transmission with a gene frequency of 0.14 and a penetration of 48 percent.

Evidence favoring that a contribution of the fetal genotype to the occurrence of preeclampsia has led to the assumption of a combined mode of transmission. For example, Cooper et al. ${ }^{59}$ reported a raised incidence of eclampsia in women born of eclamptic pregnancy (37 percent) as compared with women having a sister or brother born of eclamptic pregnancy (14 percent). Liston et al. ${ }^{60}$ studied six simple Mendelian models of inheritance by comparing theoretical expectations and data from published family studies. They concluded that only one of the genetic models considered was consistent with the observed incidence values for relatives of index cases, namely the one in which both mother $(\mathrm{a}, \mathrm{a})$ and fetus $(\mathrm{a}, \mathrm{a})$ must express the same recessive gene to confer susceptibility. Another argument against a simple Mendelian genetic model acting only in the mother is provided by twin studies. Thornton et al. ${ }^{61}$ could only demonstrate the occurrence of preeclampsia in one of the identical twins, never in both twins. They concluded that preeclampsia is not a maternal autosomal recessive condition. They were not able to exclude the possibility of a maternal dominant inheritance with incomplete penetrance (upper limit 31 percent)

Linkage of preeclampsia to a susceptibility gene could be of value in the early prediction of preeclampsia. Hayward and co-workers ${ }^{62}$ searched for this linkage in two generation families in which preeclampsia was rigorously defined. Unfortunately, they did not find evidence for linkage of preeclampsia to the HLA region or to several genes implicated in the pathogenesis of hypertension.

\section{Vascular-mediated factors}

Diseases, clinical or subclinical, which interfere with the function of endothelial cells, are known to increase the risk of preeclampsia. These conditions are able to act at three levels in the cascade leading to preeclampsia: defective placentation, placental ischemia and endothelial cell dysfunction (Fig. 2). Table 2 lists these risk factors together with their risk ratio ${ }^{63}$. The common factor is their interference with the condition/function of the maternal vasculature. Some of them, such as diabetes mellitus and chronic hypertension, are well-known risk factors. Recently Dekker et al. ${ }^{64}$ reported that more than 50 percent of the patients with a history of severe early-onset preeclampsia showed hidden abnormalities in metabolism and/or hemostasis. These abnormalities consisted of protein $S$ deficiency, activated protein $\mathrm{C}$ resistance, the presence of anticardiolipin antibodies in the peripheral blood, and hyperhomocystinemia. All of these conditions facilitate the activation of the clotting cascade. Prepregnant identification of these abnormali- 


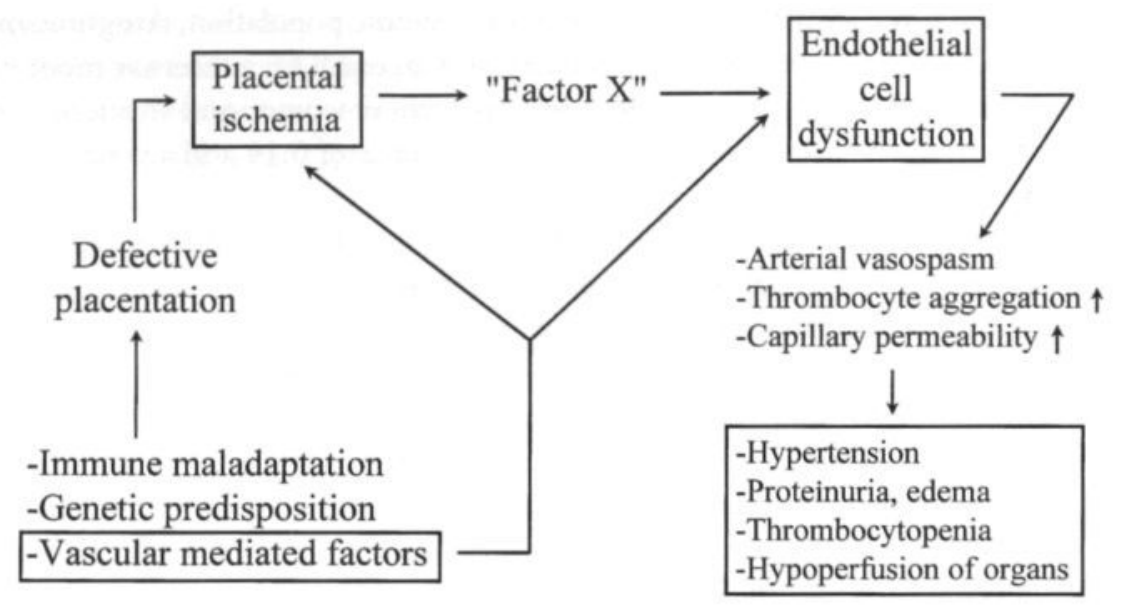

Figure 2. Postulated pathogenesis of preeclampsia.

Table 2. Vascular mediated factors involved in the pathogenesis of preeclampsia (risk ratio)

Chronic hypertension

Diabetes mellitus

Autoimmune disorders, e.g. SLE, antiphospholipid syndrome

Chronic renal insufficiency (serum creatinine $>1.4 \mathrm{mg} / \mathrm{dl}$ )

Subclinical abnormalities in metabolism and/or hemostasis

giving rise to thrombophilia (see text)
$10: 1$

2:1

10:1

20:1

increased

ties in patients with a history of preeclampsia offers the possibility of anticipating an increased recurrence risk.

In our follow-up study (at least five months postpartum), we found approximately a 10 percent lower plasma volume in patients with a history of preeclampsia as compared with controls ${ }^{65}$. Also in a normotensive subgroup the subnormal plasma volume was paralleled by a lower plasma concentration of atrial natriuretic peptide (ANP) and higher plasma concentrations of aldosterone as compared with controls. The effective renal plasma flow (ERPF) was lower and the filtration fraction higher in ex-preeclamptics as compared with controls. Both the observed differences in hormone levels and the lower renal blood flow supported the existence of a hypovolemic state in ex-preeclamptics as compared with controls. These observations support some yet unidentified vascular-mediated risk factor to be in- 
volved in the pathogenesis of preeclampsia, which has as a common effect a constricted plasma volume compartment. Obviously, a prospective study is needed to determine whether this interrelation is causal or coincidental.

The missing link

It is an attractive hypothesis that preeclamptic women have a circulating factor released by the ischemic placenta, that interferes with endothelial function, giving rise to vasoconstriction, enhanced capillary permeability and activation of the clotting cascade. Both Rodger et al. ${ }^{66}$ and Tsukimori et al. ${ }^{67}$ demonstrated that the sera from preeclamptic women damage human endothelial cells in-vitro. By measuring the release of radiolabeled chromium from the cells into the culture medium, Tsukimori et al. ${ }^{68}$ described a selective affinity of this serum factor to endothelial cells, and not to vascular smooth muscle cells or fibroblasts. Smárason and co-workers ${ }^{69}$ could only demonstrate a toxic effect of plasma and not serum, to endothelial cells of women with preeclampsia as compared to controls. The explanation for this discrepancy lies probably in the fact that they assessed a different aspect of endothelial cell function namely spontaneous proliferation by measuring tritiated thymidine incorporation.

What is behind this so-called "factor X"? ${ }^{70} \mathrm{~A}$ current concept is the formation of oxygen free radicals caused by immune maladaptation, and subsequent activation of (decidual) lymphoid cells, and placental ischemia. These extremely reactive oxygen species are assumed to play an important role in many physiologic reactions. However, when their production exceeds the neutralization capacity of free radical scavengers, a condition of imbalance is created which favors the formation of lipid peroxides from unsaturated fatty acids. Oxygen free radicals as well as lipid peroxides are toxic for endothelial cells ${ }^{71}$. Together with oxygen free radicals and cytotoxic cytokines, proteases such as elastase are released by activated neutrophils. These proteases are able to destroy the integrity of endothelial cells, vascular basement membrane and subendothelial matrix. Plasma neutrophil elastase is elevated in preeclamptic patients when compared to normotensive controls, and correlates with the elevated levels of the von Willebrand factor ${ }^{72}$.

Alternatively, changes in lipid metabolism in preeclamptic women could represent "factor X". Lorentzen and co-workers ${ }^{73}$ found that sera from women with preeclampsia induced accumulation of triglycerides in endothelial cells, which interfered with endothelial cell function as indicated by reduced prostacyclin release. The same group demonstrated that both an increased ratio of free fatty acids to albumin and an enhanced lipolytic activity of sera from women with preeclampsia resulted in a higher endothelial uptake of free fatty acids, which are further esterified into triglycerides ${ }^{74}$.

Smárason and co-workers ${ }^{75}$ proposed that a likely candidate for "factor $\mathrm{X}$ " is syncytiotrophoblast, which is shed from the microvillous membrane into the mater- 
nal circulation. They found that syncytiotrophoblast microvillous (STBM) membrane suppressed the growth of cultured human derived umbilical endothelial cells (HDUEC) and disrupted the cell monolayer. Unfortunately, there was no difference in activity between the STBM membrane preparations from preeclamptic and normal placentas. Their group already showed that trophoblast deportation was markedly increased in preeclampsia ${ }^{76}$. Next, they hypothesized a quantitative rather than a qualitative difference, suggesting an increased release of active substance in preeclamptic women.

\section{CONCLUSIONS}

Pregnancy complicated by preeclampsia can be a life-threatening event for the fetus and the mother. Central to the pathogenesis of preeclampsia are placental ischemia secondary to an initial defective placentation, and generalized endothelial cell dysfunction at the end of the cascade leading to preeclampsia. The link between these two, the toxic circulating "factor X", and the multifactorial origin of this defective placentation are subject to current research. Impaired development of immunologic tolerance may lead to an activated immune system during trophoblast invasion and subsequent defective placentation. Genetic predisposition, the possession of a susceptibility gene, and maternal vascular pathology could be modulating factors in this process. The recent finding of latent abnormalities in hemostasis and metabolism (giving rise to thrombophilia), and volume homeostasis in patients with a history of preeclampsia is suggestive for an important role of these vascular-mediated factors in the pathogenesis of preeclampsia. Prepregnant identification of these abnormalities in patients with a history of preeclampsia may enable the clinician to foresee the recurrence risk and allow him to institute preventive measures before pregnancy or in the subclinical phase of the disease. 


\section{CHAPTER Peripheral hemodynamics and renal function in relation to the menstrual cycle}

E. van Beek, A.J.H.M. Houben*, P.N. van Es*, C. Willekes**,

E.C.C.M. Korten, P.W. de Leeuw*, L.L.H. Peeters

Departments of Obstetrics and Gynecology, and Internal Medicine*, University Hospital Maastricht, and department of Physiology**, Cardiovascular Research Institute Maastricht, The Netherlands.

Clin Sci 1996; 91: 163-168 
1. The objective of this study was to investigate whether the luteal phase of the menstrual cycle differs from the follicular phase by the development of a state of general vascular relaxation.

2. Once in the follicular and once in the luteal phase of the menstrual cycle, we measured by non-invasive techniques: arterial blood pressure (by finger blood pressure measurements), vascular tone (by pulse-wave velocity and plethysmography), blood flow to skin (by laser-Doppler), blood flow to forearm (by plethysmography) and blood flow to kidneys (by para-aminohippurate clearance), and the glomerular filtration rate (by inulin clearance). The data points obtained in the luteal phase were compared with those in the follicular phase by non-parametric tests.

3. Arterial blood pressure, vascular tone and the blood flows to the forearm and kidneys were comparable in the two phases of the menstrual cycle. In contrast, the blood flow to the skin was consistently lower, and the glomerular filtration rate higher in the luteal phase of the menstrual cycle.

4. The results of the present study do not support our hypothesis of a general vascular relaxation in the luteal phase of the menstrual cycle. The lower skin flow in the luteal phase may be an adaptation needed to ensure the higher core temperature of $0.3-0.5^{\circ} \mathrm{C}$ in the luteal phase. The higher glomerular filtration rate was in most cases paralleled by a higher renal blood flow in the luteal phase. This suggests that the higher glomerular filtration rate is secondary to a selective vasorelaxation of the afferent renal arterioles.

\section{INTRODUCTION}

Several studies have shown that systemic hemodynamics and volume homeostasis in women are related to the menstrual cycle. The mean arterial blood pressure (MAP) was found to be lower ${ }^{7}$, and the plasma renin activity and creatinine clearance higher in the luteal phase (LP) than in the follicular phase (FP) of the menstrual cycle ${ }^{10,15}$. In addition, the osmotic thresholds for thirst and vasopressin release were reported to have decreased in the $\mathrm{LP}^{8}$. From a theoretical point of view, all these changes could be viewed as compensatory mechanisms triggered by a decline in filling pressure of the arterial tree ${ }^{77}$. Such a state may develop in a healthy person, when the arterial/arteriolar tone slackens.

The steroid environment in the LP differs from that in the FP primarily by the presence of progesterone in the former. Both progesterone ${ }^{78,79}$ and $17 \beta$-estradiol $80 \mathrm{can}$ independently induce vascular relaxation. However, these data have been obtained either from animal studies ${ }^{78}$ or in vitro using human placental arteries 
and veins ${ }^{79}$. Moreover, it is incorrect simply to extrapolate these results to the complex endocrine environment of the LP.

Our objective was to investigate in vivo, whether the hormonal environment in the LP induces a general vascular relaxation in humans. To this end we measured the differences between the LP and FP in venous and arterial tone and in the blood flow to three vascular beds: skin, forearm and kidney. We also determined the glomerular filtration rate (GFR) and filtration fraction.

\section{SUBJECTS AND METHODS}

Experiments were performed in nine healthy volunteers ( $<40$ years of age) with a regular menstrual cycle of $28 \pm 2$ days and evidence of ovulation, indicated by a mid-luteal serum progesterone concentration $>5 \mathrm{ng} / \mathrm{ml}{ }^{81}$. All participants gave written informed consent and the study was approved by the hospital's medical ethics committee. Measurements were taken once in the FP, between the 4th and 10 th days, and once in the LP, between the 20th and 24th days of cycle. The subjects' characteristics are listed in Table 1. The two measurements were taken randomly. This implied that four subjects started the measurements in the FP whereas five subjects started in the LP.

\section{Methods}

All investigations were performed in a temperature-controlled room $\left(24-25^{\circ} \mathrm{C}\right.$.) and at the same time of day. The subjects were asked not to smoke, not to drink beverages containing caffeine or alcohol on the day of measurements and not to eat during the last three hours before the measurements. We took precautions to minimize external disturbances during the measurements. The measurements were started after an acclimatization period of at least 30 minutes; they were performed with the subject in supine position and the arm under investigation at heart level. Before the acclimatization period a 20 gauge catheter was inserted into a vein of the left forearm for infusion of para-aminohippurate sodium (PAH) and inulin (see renal hemodynamics).

The arterial wall distensibility was assessed by measuring the pulse-wave velocity. The pulse-wave velocity is based on the time needed for an arterial pulse to travel over a standardized distance in a given artery (transit time, $\mathrm{ms}$ ). The transit time provides information on the average elastic state of the artery over that particular distance ${ }^{82}$. The transit time in this study was taken as the mean of 3-5 successive heart beats. A change in transit time within a subject provides an accurate estimate for a change in the overall arterial distensibility in that subject, because the length of the arterial tree remains constant. The opening of the aortic valves (R-top in 
ECG signal) was taken as starting point. The onset of the distension waveform, assessed by Vessel Wall Tracking, at a defined site on the right common femoral artery, was taken as the end-point. Vessel Wall Tracking is an ultrasound technique which enables the transcutaneous measurement of the vessel diameter and diameter change during consecutive heart beats ${ }^{82,83}$.

Renal hemodynamics, i.e. effective renal plasma flow (ERPF) and GFR, were measured by continuous infusion of PAH (MSD, West Point, PA, U.S.A.) and inulin (Inutest; Laevosan Gesellschaft, Linz, Austria), respectively. After the bolus injection, a continuous infusion was initiated using a syringe pump (Terumo SYC-521; Terumo Corporation, Tokyo, Japan) ${ }^{84}$. After an equilibration period of $120 \mathrm{~min}$, a total of four blood samples were collected in heparinized glass tubes (Monoject; Sherwood Medical, St Louis, MO, U.S.A.) at ten min intervals. Once blood for measurements of progesterone and $17 \beta$-estradiol was withdrawn in a vacutainer glass tube (Monoject). The blood samples were centrifuged at $4{ }^{\circ} \mathrm{C}$ for $10 \mathrm{~min}$ at $1500 \mathrm{~g}$ directly after sampling. All plasma and serum samples were stored at $-20^{\circ} \mathrm{C}$ before assay. In the plasma samples both $\mathrm{PAH}$ and inulin were measured as detailed elsewhere ${ }^{85,86}$. Serum progesterone and $17 \beta$-estradiol were measured using commercially available radioimmunoassay kits: RSL direct progesterone and RSL direct 17 $\beta$-estradiol kit (ICN Biomedicals, CA, U.S.A.), respectively. The clearances of PAH and inulin were calculated by dividing the product of infusion rate and infusate concentration by the mean of plasma PAH and plasma inulin concentration, respectively. Assuming an extraction ratio for PAH of $90 \%{ }^{87}$, we calculated renal plasma flow and renal blood flow (RBF) using the formulas: 10/9 x ERPF and 10/9 x ERPF/(1-Hematocrit), respectively. We calculated renal vascular resistance using the formula: (MAP/RBF) $x 80000$. The filtration fraction was obtained by taking the ratio of the inulin clearance (GFR) and the renal plasma flow.

We determined thermoregulatory skin perfusion using laser-Doppler fluxmetry (Periflux PF3; Perimed, Järfälla, Sweden), with probe PF 308, wide band (12 $\mathrm{kHz}$ ) mode, and time constant $0.2 \mathrm{~s}$. Measurements were performed at the volar side of the endphalanx and dorsal side of the interphalanx of the right third finger. The mean value of two minutes recording was used for off-line analysis. Flux values were expressed in arbitrary units $(\mathrm{Pu})$; calibrated to the periflux motility standard. Biological zero values, obtained during arterial occlusion, were subtracted from the measured laser-Doppler fluxmetry levels. Skin temperature was measured at the dorsal side of the interphalanx of the right fourth finger using a Hewlett Packard 78214C monitor (Hewlett Packard, Böblingen, Germany). 
Total forearm blood flow (FBF) was determined using ECG-triggered straingauge venous occlusion plethysmography (Periflow; JSI; Beerse, Belgium) and has been described in detail elsewhere ${ }^{88}$. In short, the cuff for venous occlusion was placed just above the elbow. A mercury strain-gauge was placed around the arm, $3-5 \mathrm{~cm}$ distal to the lateral humeral epicondyle. The hand circulation was stopped by inflating a wrist cuff to suprasystolic pressure, starting one minute before each FBF measurement. Hence, FBF represents predominantly muscle blood flow ${ }^{89}$. FBF was measured over a period of four minutes. The mean of the last two minutes was used for calculations. A flow curve encompassed the time interval of five heartbeats, during which venous occlusion $(50 \mathrm{mmHg})$ was applied over three heartbeats. Thus, on average, we measured 12 inflow curves $/ \mathrm{min}$.

MAP $(\mathrm{mmHg})$ and heart rate (beats/min) were measured non-invasively over a one minute period on the third finger of the left hand using a blood pressure monitor (Finapres; Ohmeda, Englewood, CO, U.S.A.).

Venous compliance was determined as described elsewhere ${ }^{88}$. To measure venous pressure and to take blood samples, we inserted a 20 -gauge catheter into a vein of the right forearm. A pressure transducer was placed $5 \mathrm{~cm}$ below the sternal angle. Intravenous pressure was measured with a Hewlett Packard 78205C pressure monitor. Forearm volume changes were measured using mercury strain-gauge venous occlusion plethysmography as described above. Measurements began 30 minutes after venepuncture. First, the upper arm cuff was inflated to a cuff pressure of $25 \mathrm{mmHg}$ and was kept inflated for three minutes. This time interval was chosen to reach a steady state in arm volume and pressure. Thereafter, the cuff was deflated for two minutes to minimize accumulation of interstitial fluid due to capillary filtration. The concomitant changes in volume and intravenous pressure during each cuff pressure step were derived from the values measured just before and after deflation of the cuff. Subsequently, the same procedures were followed to obtain volume/pressure ratios during cuff pressures of $30,35,40$ and $50 \mathrm{mmHg}$. Venous compliance was defined as the slope of the relationship, calculated by linear regression, of the forearm volume $(\mathrm{dV}, \mathrm{ml} / 100 \mathrm{ml})$ on the ordinate with the venous pressure $(\mathrm{dP}, \mathrm{mmHg})$ on the abscissa.

\section{Statistical analysis}

Differences between LP and FP were analyzed by the Wilcoxon matched-pairs signed-ranks test. Correlations between concomitantly measured variables in the FP and LP, and between differences in potentially related variables were tested by Spearman's rank correlation analysis. In the comparisons, a P-value of less than $5 \%$ was considered statistically significant.

We calculated detectable changes in the mean of variables $(n=9, \alpha=0.05$ and $\beta=0.10$ ), assuming a normal distribution of the data. The minimum detectable 
changes were $23 \%, 4 \%, 48 \%, 9 \%$ and $6 \%$ for the venous compliance, transit time, FBF, ERPF and GFR, respectively.

Table 1. Characteristics of subjects.

\begin{tabular}{|c|c|c|c|c|c|c|c|c|c|}
\hline \multirow[b]{2}{*}{ Subjects } & \multirow[b]{2}{*}{$\begin{array}{l}\text { Age } \\
\text { (years) }\end{array}$} & \multirow[b]{2}{*}{ Parity } & \multirow[b]{2}{*}{$\begin{array}{l}\text { Body mass } \\
\text { index }\left(\mathrm{kg} / \mathrm{m}^{2}\right)\end{array}$} & \multicolumn{2}{|c|}{$\begin{array}{l}\text { Cycle day of } \\
\text { measurement }\end{array}$} & \multicolumn{2}{|c|}{$\begin{array}{l}\text { Estradiol } \\
\text { (pg/ml) }\end{array}$} & \multicolumn{2}{|c|}{$\begin{array}{l}\text { Progesterone } \\
(\mathrm{ng} / \mathrm{ml})\end{array}$} \\
\hline & & & & FP & LP & FP & LP & FP & LP \\
\hline 1 & 32 & 0 & 21.7 & 6 & 21 & 83 & 159 & $<0.25$ & 19.7 \\
\hline 2 & 20 & 0 & 27.5 & 10 & 24 & 50 & 148 & $<0.25$ & 6.5 \\
\hline 3 & 36 & 0 & 20.7 & 10 & 20 & 273 & 99 & $<0.25$ & 12.4 \\
\hline 4 & 21 & 0 & 24.7 & 10 & 24 & 47 & 81 & $<0.25$ & 10.3 \\
\hline 5 & 33 & 0 & 22.5 & 4 & 24 & 32 & 144 & $<0.25$ & 16.4 \\
\hline 6 & 21 & 0 & 23.1 & 6 & 24 & 36 & 132 & $<0.25$ & 10.9 \\
\hline 7 & 36 & 0 & 23.1 & 7 & 23 & 16 & 79 & $<0.25$ & 7.8 \\
\hline 8 & 24 & 1 & 19.7 & 4 & 23 & 40 & 24 & 0.28 & 5.8 \\
\hline 9 & 39 & 2 & 22.9 & 5 & 24 & 62 & 83 & $<0.25$ & 8.7 \\
\hline
\end{tabular}

\section{RESULTS}

Table 1 lists the general characteristics of the study population and the serum levels of $17 \beta$-estradiol and progesterone in the FP and LP, respectively. In 7 of 9 cases serum levels of $17 \beta$-estradiol were higher in the LP than in the FP $(p=0.14)$. In both the FP and the LP the $17 \beta$-estradiol varied over a wide range. In the LP, progesterone also varied over a wide range.

Medians (and ranges) for hemodynamic and renal variables are listed in Table 2. The most relevant variables are also shown in Fig. 1. Skin blood flow and skin temperature were consistently lower, and GFR was higher in the LP. None of the other variables differed significantly between the LP and the FP. Neither the absolute values in the LP nor the magnitude of the change relative to the FP of all variables listed in Table 2 correlated with the serum level of progesterone in the LP, with the change in serum level of $17 \beta$-estradiol between FP and LP, or with the ratio of progesterone and $17 \beta$-estradiol in the LP. 
Table 2. Medians (with ranges) for hemodynamic variables and kidney function during the FP and LP of the menstrual cycle $(n=9)$. Median differences [with $95 \%$ confidence interval] are listed. Changes in LP relative to FP were evaluated using the Wilcoxon matched-pairs signedranks test. Pu, perfusion units.

\begin{tabular}{|c|c|c|c|c|}
\hline & FP & LP & $\begin{array}{l}\text { Median } \\
\text { difference }\end{array}$ & P-value \\
\hline $\begin{array}{l}\text { Venous compliance } \times 100 \\
\left(\mathrm{ml} 100 \mathrm{ml}^{-1} \mathrm{mmHg}^{-1}\right)\end{array}$ & $\begin{array}{l}5.5 \\
(4.8-8.0)\end{array}$ & $\begin{array}{l}6.6 \\
(4.4-8.8)\end{array}$ & $\begin{array}{l}+0.7 \\
{[-1.7 \leftrightarrow+3.0]}\end{array}$ & 0.33 \\
\hline $\begin{array}{l}\text { Transit time } \\
\text { (ms) }\end{array}$ & $\begin{array}{l}207 \\
(194-217)\end{array}$ & $\begin{array}{l}202 \\
(195-221)\end{array}$ & $\begin{array}{l}0 \\
{[-13 \leftrightarrow+7]}\end{array}$ & 0.78 \\
\hline $\begin{array}{l}\text { Mean arterial pressure } \\
\qquad(\mathrm{mmHg})\end{array}$ & $\begin{array}{l}87 \\
(75-97)\end{array}$ & $\begin{array}{l}87 \\
(61-93)\end{array}$ & $\begin{array}{l}0 \\
{[-13 \leftrightarrow+3]}\end{array}$ & 0.18 \\
\hline $\begin{array}{l}\text { Heart rate } \\
\text { (beats/min) }\end{array}$ & $\begin{array}{l}61 \\
(49-71)\end{array}$ & $\begin{array}{l}64 \\
(55-75)\end{array}$ & $\begin{array}{l}+6 \\
{[-7 \leftrightarrow+7]}\end{array}$ & 0.31 \\
\hline $\begin{array}{l}\text { Laser Doppler fluxmetry } \\
\text { (Pu) }\end{array}$ & $\begin{array}{l}54.2 \\
(16.7-124)\end{array}$ & $\begin{array}{l}29.6 \\
(9.5-59.8)\end{array}$ & $\begin{array}{l}-17.8 \\
{[-66.4 \leftrightarrow-5.8]}\end{array}$ & 0.02 \\
\hline $\begin{array}{l}\text { Laser Doppler fluxmetry } \\
\text { (Pu) }\end{array}$ & $\begin{array}{l}6.2 \\
(2.1-13.2)\end{array}$ & $\begin{array}{l}5.0 \\
(1.5-26.1)\end{array}$ & $\begin{array}{l}-0.4 \\
{[-7.9 \leftrightarrow+2.0]}\end{array}$ & 0.86 \\
\hline $\begin{array}{l}\text { Temperature }_{\sin } \\
\left({ }^{\circ} \mathrm{C}\right)\end{array}$ & $\begin{array}{l}31.4 \\
(27.6-33.5)\end{array}$ & $\begin{array}{l}27.0 \\
(24.1-32.2)\end{array}$ & $\begin{array}{l}-1.9 \\
{[-4.8 \leftrightarrow-0.6]}\end{array}$ & 0.04 \\
\hline $\begin{array}{l}\text { Forearm blood flow } \\
\qquad\left(\mathrm{ml} 100 \mathrm{ml}^{-1} \mathrm{~min}^{-1}\right)\end{array}$ & $\begin{array}{l}2.7 \\
(1.2-5.4)\end{array}$ & $\begin{array}{l}1.8 \\
(1.2-2.4)\end{array}$ & $\begin{array}{l}-0.4 \\
{[-1.0 \leftrightarrow+0.4]}\end{array}$ & 0.10 \\
\hline $\begin{array}{l}\text { Glomerular filtration rate } \\
\text { (ml/min) }\end{array}$ & $\begin{array}{l}112 \\
(98-129)\end{array}$ & $\begin{array}{l}123 \\
(105-129)\end{array}$ & $\begin{array}{l}+7 \\
{[0 \leftrightarrow+11]}\end{array}$ & 0.03 \\
\hline $\begin{array}{l}\text { Renal blood flow } \\
\text { (ml/min) }\end{array}$ & $\begin{array}{l}852 \\
(670-1006)\end{array}$ & $\begin{array}{l}889 \\
(666-1062)\end{array}$ & $\begin{array}{l}+24 \\
{[-26 \leftrightarrow+110]}\end{array}$ & 0.17 \\
\hline $\begin{array}{l}\text { Renal vascular resistance } \\
\qquad\left(\text { dyn } \mathrm{s} \mathrm{cm}^{-5}\right)\end{array}$ & $\begin{array}{l}8955 \\
(7131-10420)\end{array}$ & $\begin{array}{l}7499 \\
(6291-10575)\end{array}$ & $\begin{array}{l}-376 \\
{[-2040 \leftrightarrow+374]}\end{array}$ & 0.21 \\
\hline $\begin{array}{l}\text { Filtration fraction } \\
\text { (GFR/RPF) }\end{array}$ & $\begin{array}{l}0.21 \\
(0.17-0.27)\end{array}$ & $\begin{array}{l}0.22 \\
(0.19-0.30)\end{array}$ & $\begin{array}{l}+0.01 \\
{[0 \leftrightarrow+0.02]}\end{array}$ & 0.31 \\
\hline
\end{tabular}




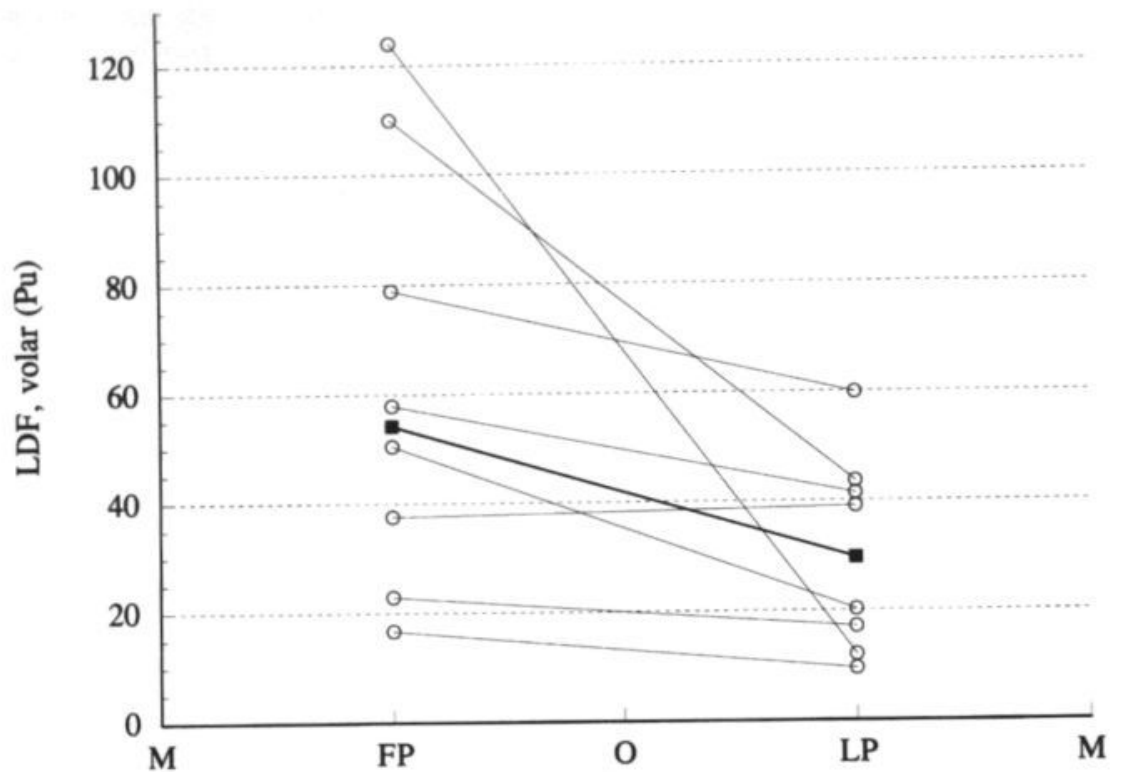

(a)

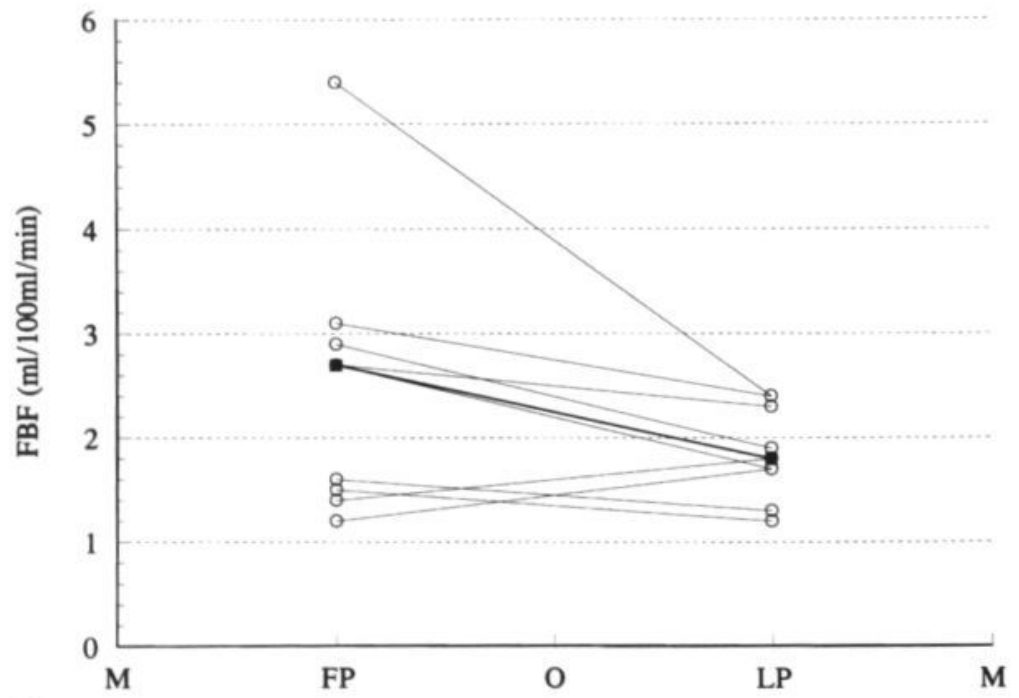

(b)

Figure 1. Skin blood flow [measured by laser-Doppler fluxmetry (LDF)] (a), FBF (b) GFR (c) and RBF (d) during the menstrual cycle. Each pair of open circles represents one subject, a filled square represents the median of that menstrual phase. FP, follicular phase; LP, luteal phase; $M$, menstrual period; $\mathrm{O}$, ovulation. 


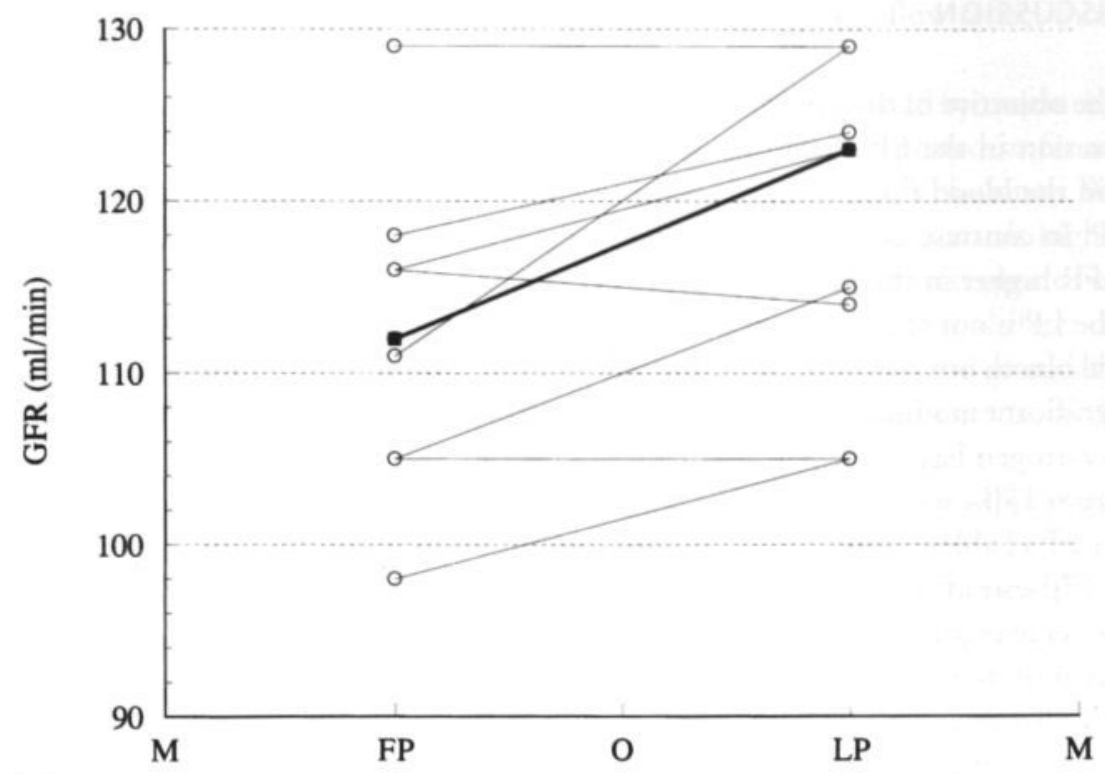

(c)

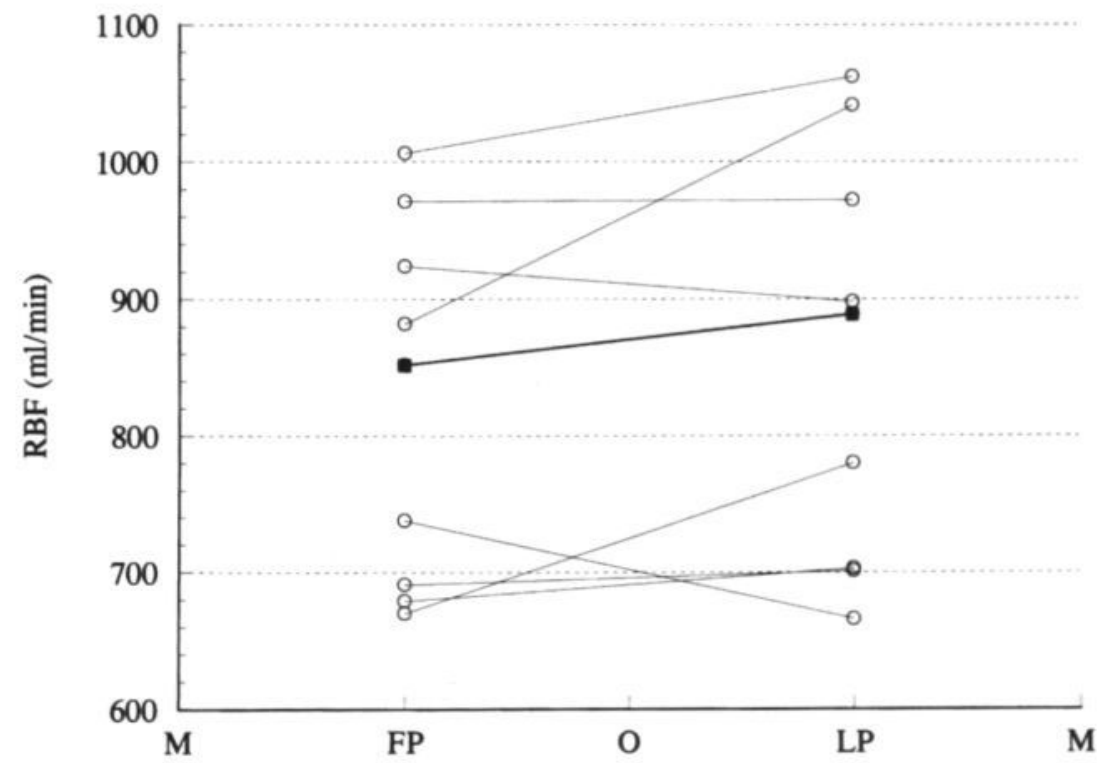

(d)

\section{Continued figure 1.}




\section{DISCUSSION}

The objective of this study was to determine whether there are signs of vascular relaxation in the LP of the menstrual cycle. Arterial blood pressure, vascular tone and the blood flows to the forearm and kidneys were comparable in the LP and FP. In contrast, skin thermoregulatory blood flow was consistently lower, and the GFR higher in the LP of the menstrual cycle.

The LP is not only characterized by the appearance of progesterone in the peripheral blood, but also by a highly variable serum level of $17 \beta$-estradiol, known to be a significant modulator of vascular tone. Most studies on vascular effects in response to estrogen have shown a vasodilatory action ${ }^{80}$. In our study the luteal value for serum $17 \beta$-estradiol was higher in 7 out of 9 subjects when compared with that in the FP (Table 1). We did not find a correlation between the change in serum level of $17 \beta$-estradiol between the two phases, and all the hemodynamic and renal variables measured.

Based on reported studies ${ }^{78-80}$, we expected to see an increase in both the venous compliance and arterial distensibility leading to a fall in pulse-wave velocity and a rise in transit time, respectively. However, our measurements suggested that neither the venous compliance nor the arterial distensibility had changed consistently in the LP. Other studies investigating venous distensibility during the menstrual cycle, show conflicting data ${ }^{90,91}$. The reproducibility of pulse-wave velocity measurements can be adversely affected by changes in arterial blood pressure ${ }^{92}$. Because of the lack of consistent change in MAP in our study, we did not correct for coincidental fluctuations in blood pressure.

Vasodilatation in response to a fall in arteriolar tone can be expected to give rise to hyperperfusion in muscle and skin tissue for example. Instead, we found a consistent reduction in skin thermoregulatory blood flow and a trend towards lower perfusion rates in forearm muscle during the LP. Other investigators have not found a consistent change in FBF either ${ }^{93-96}$. Observed changes in skin blood flow during the menstrual cycle are conflicting; varying between no change ${ }^{93,97,98}$, an increase in LP 99,100 and a decrease in LP ${ }^{101,102}$. The fall in skin thermoregulatory blood flow as observed in the present study is possibly related to the well-known 0.3-0.5 ${ }^{\circ} \mathrm{C}$ increase in core temperature during the LP. This increase in core temperature may result from either a raised metabolic rate or an increase in thermal body insulation. It follows that the higher body temperature is probably an intended effect in response to a rise in the set-point of the thermoregulatory center in the medulla oblongata. This effect can be obtained by a fall in thermoregulatory skin flow (arteriovenous shunt flow) ${ }^{101}$. The blood flow to the skin of the finger tip is more influenced by thermoregulation than is the case on the dorsal side of the finger, where arteriovenous shunts are relatively scarce. The consistent fall in just the fin- 
ger tip flow supports the view that the observed changes in skin flow reflect those in thermoregulation.

To our knowledge the cyclicity in the GFR has never been evaluated by measuring serum inulin clearance; in other words, all data on this topic reported so far are based on GFR markers such as creatinine clearance or ${ }^{51} \mathrm{Cr}$-EDTA clearance. We showed a $10 \%$ rise in median GFR in conjunction with a trend towards higher RBF and lower renal vascular resistance in the LP. Other investigators reported either a similar cyclicity ${ }^{9-11,103}$ or lack of cyclicity ${ }^{104}$, probably due to differences in methodology. The combined increase in both GFR and RBF in five of six subjects supports the view that the mechanism of this increase is afferent arteriolar dilation. However, this requires further study.

The observed blood flow patterns to muscle, skin and kidney do not support our hypothesis of a general vascular relaxation in the LP. Since we studied the blood flows to only three vascular beds, it was impossible to draw conclusions about changes in total peripheral resistance with the menstrual cycle. However, it has been demonstrated that total peripheral resistance decreases in the LP of the menstrual cycle ${ }^{105}$.

A limitation of these in vivo experiments is the relatively high variability resulting from the combination of biological variation (2-week interval) and the use of indirect measurement techniques. In our institute this combined variability is associated with coefficients of variation ranging from 10 to $25 \%{ }^{88,106}$. Since the measurements were paired and performed by the same investigator, we expected to minimize this problem. Table 2 shows the $95 \%$-confidence intervals of the median differences for each of the measured variables. Although some variables are characterized by relatively wide intervals (modest power), others show relatively narrow intervals. The minimum detectable changes mentioned above do support these findings and illustrate, in combination with the actual measured changes, the possibility of a type II error.

In conclusion, the present data do not support the concept of a general vascular relaxation during the LP of the menstrual cycle. Thermoregulatory blood flow in the skin was most probably diminished in LP, in order to raise the core temperature. Post-ovulatory vasodilatation of afferent renal arterioles could be responsible for the raised GFR, and possibly the endocrine environment of the LP induces selective vasorelaxation in certain vascular beds.

\section{ACKNOWLEDGMENTS}

We thank Hubert J.A. Schouten for his valuable contributions to the statistical analysis. 



\section{$4 \begin{aligned} & \text { CHAPTER } \\ & \text { Body fluid volume changes }\end{aligned}$ during the menstrual cycle}

Erik van Beek, Alfons J.H.M. Houben*, Timo H.A. Ekhart*, Paul M.H. Schiffers ${ }^{* * *}$, Emile C. Cheriex**, Peter W. de Leeuw*, Louis L.H. Peeters

Departments of Obstetrics and Gynecology, Internal Medicine*, and Cardiology**, University Hospital Maastricht and department of Pharmacology***, University of Maastricht, Maastricht, The Netherlands.

Submitted 
This study was designed to investigate whether total peripheral resistance decreases and plasma volume and extra-cellular fluid volume increase in the luteal phase of the menstrual cycle relative to the follicular phase.

We measured in both luteal and follicular phase of the menstrual cycle, cardiac output (echocardiography), blood pressure (semi-automatic oscillometry), plasma volume ( ${ }^{125}$ I-human albumin dilution technique), extra-cellular fluid volume (sodium bromide dilution technique) and the plasma levels of their regulatory neurohormones (renin-angiotensin-aldosterone system, ANP and catecholamines). Measurements were performed in two consecutive menstrual cycles during a lowand during a high-salt diet. The data points obtained in the luteal phase were compared with those in the follicular phase of the same menstrual cycle. The follicular phases of the two consecutive menstrual cycles with different salt intake were compared with each other. All comparisons were performed by non-parametric tests. Switching to a high-salt diet led to a lower activity of the renin-angiotensinaldosterone system and a lower plasma norepinephrine concentration, together with a higher plasma atrial natriuretic peptide concentration, body weight and extra-cellular fluid volume. Only in the high-salt menstrual cycles, we observed in the luteal phase relative to the follicular phase of the menstrual cycle, a lower total peripheral resistance and a higher renin-angiotensin-aldosterone system activity together with a trend towards a lower ANP. These changes were not paralleled by a concomitantly higher extra-cellular fluid volume or plasma volume.

These data suggest a lower arterial tone in the luteal phase of the menstrual cycle, a condition that triggers endocrine changes consistent with arterial underfill.

\section{INTRODUCTION}

In human pregnancy plasma volume (PV) increases by approximately $40 \%{ }^{107}$. Nowadays, it is widely accepted that the primary trigger for changes in blood volume during pregnancy is initial systemic arterial vasodilation with relative underfilling of the arterial circulation ${ }^{108}$. Reported hemodynamic changes in the menstrual cycle mimic hemodynamic adaptations seen in early pregnancy. These cyclic changes consist of a slight decrease in mean arterial pressure (MAP) ${ }^{7}$, and are paralleled by a rise in plasma renin activity ${ }^{15}$, a rise in glomerular filtration rate (GFR) ${ }^{12}$ and effective renal plasma flow (ERPF) ${ }^{109}$, and a downward reset of osmotic thresholds for thirst and vasopressin release in the luteal phase (LP) ${ }^{8}$. Whether the latter cyclic changes in volume homeostasis are also secondary to systemic arterial vasodilation with a compensatory expansion of the blood volume, is unknown. 
The present study was designed to test the hypothesis that, compared to the follicular phase (FP), total peripheral resistance (TPR) has decreased while PV and extracellular fluid volume (ECFV) have increased in the LP of the menstrual cycle. To this end, we measured the following parameters in both phases of the menstrual cycle: TPR, blood pressure (BP), PV, ECFV and their regulatory neurohormones (active plasma renin concentration (APRC), angiotensin II (AngII), aldosterone (Aldo), atrial natriuretic peptide (ANP) and catecholamines). Since changes in these parameters are determined, in part, by sodium intake, we performed our experiments during both a low- and a high-salt diet.

\section{MATERIALS AND METHODS}

\section{Subjects}

Experiments were performed in 29 healthy volunteers (mean age: $22 \pm 4$ (SD) years; mean body mass index: $22 \pm 3(\mathrm{SD}) \mathrm{kg} / \mathrm{m}^{2}$ ) with a history of regular menstrual cycles ( $29 \pm 3$ days). All participants gave written informed consent and the study was approved by the hospital's medical ethics committee. The investigation conforms with the principles outlined in the Declaration of Helsinki.

\section{Protocol}

A total of four measurements were performed divided over two consecutive menstrual cycles, in each cycle once in the follicular phase (FP), between the third - and seventh day, and once in the LP, between the 20th - and 24 th day. During one cycle the subjects used a sodium-restricted diet of $50 \mathrm{mmol} /$ day and during the other a high-sodium diet of $220 \mathrm{mmol} /$ day. They started the diet seven days before each measurement. Each subject was randomized for type of diet to start with. Accordingly, half of the subjects started with the low-salt diet, and the other half with the high-salt diet. They collected a 24 -hour urine sample for determination of sodium and creatinine contents the day before each measurement. Compliance with the diet was assessed by the 24-hour sodium excretion. Completeness of urine collection was verified by 24 -hour creatinine excretion. Measurements started at 8.00 a.m., after an overnight fast, and ended at 1.00 p.m.. The subjects were asked not to smoke and not to drink beverages containing caffeine or alcohol, and to drink water only, on the day of the measurements. Experimental conditions were similar on each of the four study days. The measurements were performed with the subject in supine position and were started after an acclimatization period of at least $30 \mathrm{~min}$. The subjects were asked to drink a solution containing sodiumbromide $(20.0 \mathrm{ml}, 150 \mathrm{mmol} / \mathrm{l})$ so as to quantify the size of the ECFV. Just before the acclimatization period a 20 -gauge catheter was inserted into a vein of the left forearm in order to take blood samples. Blood samples were collected for measurement of 
hematocrit $(\mathrm{Ht})$, osmolality, albumin, progesterone, 17 $\beta$-estradiol, catecholamines (norepinephrine, epinephrine and dopamine), APRC, AngII, Aldo, ANP and bromide. Blood samples for the measurement of the latter hormones and bromide were centrifuged at $4^{\circ} \mathrm{C}$ for $10 \mathrm{~min}$ at $1500 \mathrm{~g}$, immediately after sampling, and stored deep-frozen before assay. The other blood samples were sent to the routine hospital laboratories for direct determination. Besides sampling, echocardiographic measurements were performed, followed by determination of the PV.

\section{Measurements}

Plasma volume (CV 4.2\%) was measured, at approximately the same time in the morning, as follows ${ }^{110}$. Five $\mu \mathrm{Ci}^{125} \mathrm{I}$-human albumin, dissolved in $1 \mathrm{ml}$ saline, was given intravenously at time zero (total body dose of irradiation $=0.04 \mathrm{mSv}$ ). Before the bolus injection and again after 10,20 and $30 \mathrm{~min}$, blood samples $(5 \mathrm{ml})$ were drawn to determine the volume specific plasma radioactivity. The virtual volume specific plasma radioactivity at time zero, was calculated by extrapolation using the three serially obtained volume specific plasma radioactivity data points after bolus injection. PV was calculated by dividing the total injected radioactivity by the virtual volume specific plasma radioactivity at time zero, corrected for blanks.

ECFV was determined with sodium bromide on the basis of its distribution space, analogous to the above mentioned ${ }^{125}$ I-human albumin dilution for determination of $\mathrm{PV}^{111}$. Sodiumbromide $(4.50 \mathrm{mmol})$ was given orally at time zero. Prior to intake (blank value) and again after a $4 \mathrm{~h}$ equilibration period a blood sample was taken. Determination of bromide in these samples was performed by capillary gas chromatography $(\mathrm{CV} 4 \%)^{111}$. Bromide space was calculated by dividing the orally administered dose of bromide by the concentration of serum bromide after correction for blanks.

Echocardiographic measurements, as described in detail elsewhere ${ }^{112}$, were performed in semi-left lateral position. The echocardiographic recordings were made with a cross-sectional, phased array echocardiographic Doppler system (HewlettPackard Sonos 500 and 1000). Cardiac output (CO) was calculated according to the formula: $\mathrm{CO}=$ stroke volume (SV) $\mathrm{x}$ heart rate (HR). To determine SV, blood flow velocity was measured across the aortic valve. SV was calculated as aortic area $x$ aortic velocity integral. TPR was calculated by using the formula TPR $\left(\right.$ dyn.s.cm $\left.{ }^{-5}\right)=\operatorname{MAP}(\mathrm{mmHg}) \times 80 / \mathrm{CO}(\mathrm{l} / \mathrm{min})$. In this formula, the MAP was calculated as the average of three recordings during measurement of the flow across the aortic valve using a semi-automatic oscillometric device (Dinamap Vital Signs Monitor 1846, Critikon, Tampa, FL, USA). 
The hematocrit, as well as the serum osmolality and albumin were measured by standard laboratory techniques. Serum $17 \beta$-estradiol and progesterone were determined using reagent kids of DPC (DPC, Los Angeles, Cal.). These assays are based on solid-phase radioimmunoassays using coated tube technology. Sensitivity of the assays are 0.035 and $0.15 \mathrm{nmol} / \mathrm{l}$, respectively. In the measurements of these two steroids, precision is better than $5 \%$.

APRC was assessed by the IRMA-method (Nichols Institute Diagnostics, Wychen, The Netherlands) with an intra-assay coefficient of variation (CV) of $2.9 \%$ and an inter-assay $\mathrm{CV}$ of $7.6 \%{ }^{113}$. AngII was determined by radio-immunoassay (RIA) following Ph Phenyl column extraction (Amersham Int. Amersham, UK; intra-assay CV $4.6 \%$ and inter-assay CV 7.7\%) ${ }^{114}$. Aldo was assayed by means of a solid-phase protein binding RIA (Diagnostic Products Corporation, LA, USA; intra- and inter assay CV $4.3 \%$ and $6.7 \%$ resp. ${ }^{115}$. ANP was measured by a competitive protein-binding radio-immunoassay (RIA) (Nichols Institute Diagnostics) following extraction of plasma over Sep-Pak C18 columns 116 . Norepinephrine (intra-assay CV 4.5\%, inter-assay CV 8.1\%), epinephrine (intra-assay CV 4.1\%, inter-assay CV 8.6\%) and dopamine (intra-assay CV 3.9\%, inter-assay CV $7.1 \%$ ) were assessed by a sensitive fluorimetric method, in which catecholamines are concentrated from plasma by liquid-liquid extraction and derivatized with a selective fluorescent agent prior to chromatography ${ }^{117}$. For each substance, all samples from the same individual were assayed in a single run.

\section{Statistical analysis}

Only ovulatory cycles were included for analysis of the differences between LP and FP, whereas all the cycles were included for analysis of differences between the FP's. A menstrual cycle was considered ovulatory when the mid-luteal serum progesterone concentration exceeded $15 \mathrm{nmol} / \mathrm{l}^{81}$.

Differences between LP and FP in each cycle (low and high-salt diet, $n=12$ and 14 resp.), and in the FP between the two salt diet cycles $(n=25)$ were analyzed by the Wilcoxon Matched-Pairs Signed-Ranks Test. Correlations between concomitantly measured variables or between differences in potentially related variables were tested by Spearman's rank correlation analysis. In the comparisons a $P$-value of less than $5 \%$ was considered statistically significant.

We calculated detectable changes in the mean of variables $(n=14, \alpha=0.05$ and $\beta$ $=0.10$ ), assuming a normal distribution of the data. The minimal detectable changes were $7.2 \%, 11.5 \%, 3.2 \%, 10.1 \%$ and $10.7 \%$ for the PV, ECFV, MAP, $\mathrm{CO}$ and TPR, respectively. 


\section{RESULTS}

Twenty-five of the 29 subjects completed all measurements (two menstrual cycles), whereas four subjects completed the two measurements in a single menstrual cycle (three low-salt menstrual cycles, one high-salt menstrual cycle). Four of the in total 54 menstrual cycles (one low-salt menstrual cycle, three high-salt menstrual cycles) were excluded from analysis because of irregular blood loss. Fourteen of the 23 (61\%) high-salt menstrual cycles and twelve of the 27 (44\%) low-salt menstrual cycles were ovulatory.

In the high-salt cycles as compared to the low-salt cycles (FP, $n=25$ ), plasma concentration of norepinephrine was $26 \%$ (median, $P<0.001$ ) lower, and ECFV and body weight were $6 \%$ (median, $P<0.05)$ and $1 \%$ (median, $P<0.01$ ) higher, respectively. When subjects used a high-salt diet, the activity of the reninangiotensin-aldosterone (RAA)-system was lower as compared to that in a low-salt diet. Using the follicular value in low-salt cycles as $100 \%$, the following follicular figures in high salt cycles were obtained: APRC $67 \%$ (median, $P<0.001$ ), AngII $77 \%$ (median, $P<0.01$ ) and Aldo $24 \%$ (median, $P<0.001$ ). The latter was paralleled by elevated ANP levels: $119 \%$ (median, $P<0.01$ ).

Tables $1 \mathrm{~A}$ and $1 \mathrm{~B}$ list the hormonal status and urinary sodium excretion of the subjects during low- $(n=12)$ and high-salt $(n=14)$ diet, respectively. In the LP relative to the FP, serum $17 \beta$-estradiol and progesterone had increased in both low- and high-salt menstrual cycles. Catecholamines and sodium excretion did not differ between LP and FP in both low- and high-salt menstrual cycles.

Tables $2 \mathrm{~A}$ and $2 \mathrm{~B}$ list the changes in central hemodynamics and body fluid volumes in the menstrual cycle during low- $(n=12)$ and high-salt $(n=14)$ diet, respectively. During the low-salt menstrual cycles none of the measured variables changed consistently. On the other hand, during the high-salt menstrual cycles TPR and albumin had decreased in the LP by a median of $5.3 \%$ and $2.6 \%$, respectively, and osmolality tended to decrease $(P=0.06)$. None of the other variables listed in Table $2 \mathrm{~B}$ had changed consistently as a function of the menstrual cycle. The volume regulatory hormones measured in the two phases of the two menstrual cycles studied are listed in Tables 3A and 3B. In the LP of both low- and high-salt menstrual cycles the hormones of the RAA-system were elevated relative to the FP. Plasma ANP concentration was significantly lower in the LP of low-salt menstrual cycles and tended to be lower in the LP of high-salt menstrual cycles $(P$ $=0.14)$.

In both low and high-salt menstrual cycles, the change in TPR, APRC or ANP did not correlate with the change in serum progesterone -, serum 17 $\beta$-estradiol concentration, nor the ratio of serum progesterone to serum $17 \beta$-estradiol. Cyclic changes in PV, ECFV, APRC or ANP during both low- and high-salt menstrual cycles did not correlate with the concomitant changes in TPR. 
Table 1A. Hormonal characteristics and sodium excretion of subjects $(n=12)$ during the FP and LP of low-salt menstrual cycles.

\begin{tabular}{|c|c|c|c|}
\hline & FP & LP & Median difference \\
\hline 17ß-estradiol (nmol/I) & $0.10(0.08-0.12)$ & $0.32(0.27-0.36)^{\star \star}$ & $+0.22(+0.14 \leftrightarrow+0.28)$ \\
\hline Progesterone $(\mathrm{nmol} / \mathrm{l})$ & $2.1(1.3-2.3)$ & $25.3(20.4-45.0)^{\star *}$ & $+22.7(+18.6 \leftrightarrow+47.3)$ \\
\hline Norepinephrine $(\mathrm{nmol} / \mathrm{l})$ & $0.63(0.54-1.10)$ & $0.65(0.61-0.79)$ & $+0.00(-0.19 \leftrightarrow+0.13)$ \\
\hline Epinephrine (nmol/) & $0.06(0.04-0.09)$ & $0.04(0.02-0.06)$ & $-0.02(-0.04 \leftrightarrow+0.00)$ \\
\hline Dopamine (nmol/l) & $0.13(0.08-0.19)$ & $0.14(0.08-0.17)$ & $+0.01(-0.08 \leftrightarrow+0.09)$ \\
\hline Sodium excr. (mmol/24h) & $56(38-66)$ & $46(34-86)$ & $+1.0(-27 \leftrightarrow+42)$ \\
\hline
\end{tabular}

Table 1B. Hormonal characteristics and sodium excretion of subjects $(n=14)$ during the FP and LP of high-salt menstrual cycles.

\begin{tabular}{|c|c|c|c|}
\hline & FP & LP & Median difference \\
\hline 17ß-estradiol (nmol/I) & $0.10(0.06-0.12)$ & $0.36(0.26-0.45)^{\star \star}$ & $+0.28(+0.13 \leftrightarrow+0.35)$ \\
\hline Progesterone $(\mathrm{nmol} / \mathrm{l})$ & $1.6(1.2-2.6)$ & $31.2(27.5-36.0)^{\star \star}$ & $+29.0(+24.4 \leftrightarrow+38.0)$ \\
\hline Norepinephrine (nmol/l) & $0.47(0.41-0.77)$ & $0.59(0.45-0.76)$ & $+0.06(-0.08 \leftrightarrow+0.26)$ \\
\hline Epinephrine (nmol/l) & $0.06(0.05-0.09)$ & $0.04(0.03-0.09)$ & $-0.02(-0.05 \leftrightarrow+0.02)$ \\
\hline Dopamine (nmol//) & $0.13(0.09-0.22)$ & $0.14(0.07-0.24)$ & $-0.03(-0.06 \leftrightarrow+0.07)$ \\
\hline Sodium excr. (mmol/24h) & $220(172-279)$ & $200(156-244)$ & $-13(-101 \leftrightarrow+66)$ \\
\hline
\end{tabular}


Table 2A. Central hemodynamics and body fluid volumes during the FP and LP of low-salt menstrual cycles $(n=12)$.

\begin{tabular}{|c|c|c|c|}
\hline & FP & LP & Median difference \\
\hline $\operatorname{MAP}(\mathrm{mmHg})$ & $79(74-84)$ & $78(72-82)$ & $-3(-7 \leftrightarrow+3)$ \\
\hline Stroke volume ( $\mathrm{ml} / \mathrm{min})$ & $78.0(74.0-79.0)$ & $80.0(75.0-82.0)$ & $+1.0(-10.0 \leftrightarrow+4.0)$ \\
\hline Cardiac output (I/min) & $5.10(4.50-5.40)$ & $5.30(4.40-5.60)$ & $-0.05(-0.40 \leftrightarrow+0.90)$ \\
\hline TPR (dyn.s. $\mathrm{cm}^{-5}$ ) & $1338(1226-1424)$ & $1204(1067-1413)$ & $-60(-352 \leftrightarrow+171)$ \\
\hline Plasma volume (ml) & $2466(2128-2792)$ & $2542(2179-2634)$ & $-54(-202 \leftrightarrow+168)$ \\
\hline Hematocrit & $0.37(0.33-0.37)$ & $0.37(0.34-0.38)$ & $+0.00(-0.01 \leftrightarrow+0.01)$ \\
\hline ECFV (I) & $17.9(13.9-19.5)$ & $16.1(15.1-20.3)$ & $-0.1(-0.9 \leftrightarrow+1.1)$ \\
\hline Body weight (kg) & $60.3(56.1-62.7)$ & $59.9(55.9-63.7)$ & $-0.4(-0.9 \leftrightarrow+1.0)$ \\
\hline Osmolality (mosm/kg) & $281(278-282)$ & $278(275-283)$ & $-3.0(-7.0 \leftrightarrow+2.0)$ \\
\hline Serum albumin $(g / l)$ & $39.3(37.9-40.4)$ & $39.0(37.9-40.5)$ & $-0.3(-2.1 \leftrightarrow+2.3)$ \\
\hline
\end{tabular}

Table 2B. Central hemodynamics and body fluid volumes during the FP and LP of high-salt menstrual cycles $(n=14)$.

\begin{tabular}{|c|c|c|c|}
\hline & FP & LP & Median difference \\
\hline MAP $(\mathrm{mmHg})$ & $82(77-85)$ & $78(75-83)$ & $-1(-4 \leftrightarrow+1)$ \\
\hline Stroke volume $(\mathrm{ml} / \mathrm{min})$ & $76.0(69.0-84.5)$ & $78.0(68.5-81.3)$ & $-0.5(-4.0 \leftrightarrow+5.0)$ \\
\hline Cardiac output (Vmin) & $4.70(4.50-5.25)$ & $4.85(4.40-5.28)$ & $-0.05(-0.5 \leftrightarrow+0.6)$ \\
\hline TPR (dyn.s. $\left.\mathrm{cm}^{-5}\right)$ & $1336(1226-1453)$ & $1320(1178-1441)^{\star}$ & $-71(-179 \leftrightarrow-10)$ \\
\hline Plasma volume (ml) & $2514(2247-2673)$ & $2463(2168-2868)$ & $-52(-159 \leftrightarrow+244)$ \\
\hline Hematocrit & $0.35(0.33-0.37)$ & $0.35(0.34-0.36)$ & $+0.00(-0.02 \leftrightarrow+0.01)$ \\
\hline ECFV (I) & $17.4(15.1-21.4)$ & $17.7(15.5-21.3)$ & $+0.2(-1.2 \leftrightarrow+1.6)$ \\
\hline Body weight & $62.5(55.6-67.0)$ & $61.3(55.2-66.8)$ & $-0.2(-1.1 \leftrightarrow+1.4)$ \\
\hline Osmolality (mosm/kg) & $282(278-284)$ & $277(276-280)$ & $-5.0(-9.0 \leftrightarrow+2.0)$ \\
\hline Serum albumin $(g / l)$ & $38.9(36.8-40.2)$ & $37.3(36.8-38.7)^{\star}$ & $-1.0(-2.5 \leftrightarrow-0.1)$ \\
\hline
\end{tabular}


Table 3A. Neurohormones during the FP and LP of low-salt menstrual cycles ( $n=12)$.

\begin{tabular}{|c|c|c|c|}
\hline & FP & LP & Median difference \\
\hline APRC (mU/l) & $28.4(16.3-37.9)$ & $35.7(32.6-46.8)^{\star *}$ & $+9.8(+4.2 \leftrightarrow+20.4)$ \\
\hline Angll (pmol/l) & $9.1(8.1-11.2)$ & $12.1(10.2-14.6)^{\star *}$ & $+2.2(+1.0 \leftrightarrow+5.2)$ \\
\hline Aldo (nmol/l) & $270(181-470)$ & $643(589-854)^{\star *}$ & $+354(+139 \leftrightarrow+484)$ \\
\hline ANP $(\mathrm{pg} / \mathrm{ml})$ & $68.5(53.8-101.8)$ & $60.0(49.1-76.5)^{\star}$ & $-7.0(-20.0 \leftrightarrow-2.0)$ \\
\hline
\end{tabular}

Table 3B. Neurohormones during the FP and LP of high-salt menstrual cycles ( $n=14)$.

\begin{tabular}{|c|c|c|c|}
\hline & FP & LP & Median difference \\
\hline APRC $(\mathrm{mU} / \mathrm{l})$ & $18.1(10.7-24.6)$ & $26.1(15.3-37.3)^{\star \star}$ & $+7.5(+0.9 \leftrightarrow+14.8)$ \\
\hline Angll (pmol/l) & $7.8(6.1-10.8)$ & $8.6(7.1-12.6)^{\star}$ & $+1.3(+0.1 \leftrightarrow+3.1)$ \\
\hline Aldo (nmol/l) & $97.5(10.0-193)$ & $255(166-446)^{\star \star}$ & $+198(+3.0 \leftrightarrow+313)$ \\
\hline ANP $(p g / m l)$ & $111.5(52.8-147.0)$ & $85.5(67.0-102.0)$ & $-32.0(-50.0 \leftrightarrow+12.0)$ \\
\hline $\begin{array}{l}\text { Values during } \\
95 \% \text { confiden } \\
\text { strual cycle we } \\
\star \star P<0.01,{ }^{\star} \\
\text { Angll, plasma }\end{array}$ & $\begin{array}{l}\text { LP are medians anc } \\
\text { are listed in the fina } \\
\text { ed using the Wilcox } \\
1 . \text { FP, follicular phas } \\
\text { II; Aldo, plasma ald }\end{array}$ & $\begin{array}{l}\text { d interquartile ranges } \\
\text { al column. Changes ir } \\
\text { on matched-pairs sig } \\
\text { se; LP, luteal phase; } \\
\text { dosterone; ANP, atria }\end{array}$ & $\begin{array}{l}\text { 5edian differences ( } W \\
\text { nariables during the med ranks test, }{ }^{\star} P<0 \\
\text { APRC, active plasma ren } \\
\text { I natriuretic peptide. }\end{array}$ \\
\hline
\end{tabular}

\section{DISCUSSION}

The present study was designed to evaluate changes in TPR in the FP and the LP of the menstrual cycle, and to explore whether a fall in TPR is paralleled by a rise in $\mathrm{PV}$ as has been proposed for early pregnancy ${ }^{108}$. Subjects on a high-salt diet had a median 5\% fall in TPR in the LP relative to the FP. Most likely, this decrease is triggered by a fall in vascular tone which again activates volume retaining mechanisms as is suggested by the increase in levels of RAA and a trend towards a lower ANP. The fact that PV and ECFV did not increase appreciably may be related to insufficient power in this particular study (minimal detectable change $7.2 \%$ and $11.5 \%$, resp.) to detect a small rise in volume. In order to reduce the scatter in data 
points caused by variation in salt intake, we asked the subjects to adhere strictly to their salt diet. In their study, in which subjects did not use a standardized salt diet, Chapman et al ${ }^{109}$ did neither find an increase in PV in the LP of the menstrual cycle. To our opinion the PV changes with the menstrual cycle are probably too small to be discerned with currently available techniques, which do not allow serial measurements throughout the menstrual cycle.

It is tempting to speculate that the $26 \%$ higher plasma norepinephrine concentration during the low-salt diet led to a higher basal vascular tone during the two sessions in that state. A higher vascular tone during low-salt menstrual cycles may have blunted the arterial relaxation in the LP. The body weight was approximately $0.7 \mathrm{~kg}$ (median) higher during high-salt diet than during the period of low-salt intake. The concomitant larger size of ECFV suggests that the higher body weight resulted from an increase in total body water.

TPR was calculated by dividing the MAP by the simultaneously measured CO. Continuous wave Doppler measurement of flow in the aortic valve has been found to provide a reliable and reproducible estimate for stroke volume ${ }^{118,119}$. Kusumoto et al ${ }^{120}$ demonstrated that continuous wave Doppler estimates of $\mathrm{CO}$ can be used to reliably detect serial changes in $\mathrm{CO}$ in response to various interventions. Our present observations of a lower TPR in the LP confirms previous studies on this subject ${ }^{105,109,121}$. In animal studies and in-vitro ${ }^{78-80}$, both $17 \beta$-estradiol and progesterone can be held responsible for the observed decrease in TPR. Therefore, we expected to find a correlation between change in serum $17 \beta$-estradiol concentration, change in progesterone concentration and/or the ratio of serum progesterone to $17 \beta$-estradiol on the one hand and change in TPR on the other hand. The fact, that we did not find such correlations indicates that the interaction between the endocrine changes during the menstrual cycle and the subsequent hemodynamic adaptation do not follow a simple dose-response relationship.

We postulate that fluid retention leading to a small increase in ECV and PV accelerates secondary to arterial relaxation in the LP. Evidence for this accelerated fluid retention is provided by the luteal increase in RAA-system activity together with a modest fall in plasma ANP concentration. Besides these endocrine changes, other factors are to be involved since neither the changes in APRC nor that in ANP correlated with those in TPR. The increase in APRC during the LP of the menstrual cycle has been reported by several other investigators ${ }^{13-15,109}$. This increase is assumed to be a compensatory response to the natriuretic effect of progesterone ${ }^{122}$. The lack of correlation between changes in APRC and changes in serum progesterone concentration indicates that other factors contribute to the APRC rise in the LP of the menstrual cycle. The fact that changes in TPR did not correlate with changes in sex steroids as well as changes in APRC makes it difficult to identify from this study the mechanism that leads to the increased RAA-system activity. 
The concurrent decline in plasma ANP concentration during the LP suggests the physical perception of intravascular volume depletion.

In conclusion, the present study demonstrates a modest decrease in TPR in the LP of the menstrual cycle. Simultaneously, we observed an increase in RAA-system activity and a tendency to a lower plasma ANP concentration. Despite the activation of sodium and fluid retaining mechanisms, ECFV and PV did not increase appreciably in the LP of the menstrual cycle. Apparently, the stimulus of arterial relaxation, and subsequent arterial underfill, in the LP of the menstrual cycle is, compared to early pregnancy, too weak to elicit a measurable rise in ECFV and PV.

\section{ACKNOWLEDGMENTS}

We thank Hubert J. A. Schouten for his valuable contributions to the statistical analysis. 


\section{CHAPTER \\ Vascular resistance in relation to the menstrual cycle}

Erik van Beek, Alfons J.H.M. Houben*, Timo H.A. Ekhart*, Paul M.H. Schiffers**, Peter W. de Leeuw*, Louis L.H. Peeters

Departments of Obstetrics and Gynecology, and Internal Medicine*, University Hospital Maastricht, and department of Pharmacology**, University of Maastricht, Maastricht, The Netherlands.

Submitted 


\section{ABSTRACT}

This study was designed to test the hypothesis that the reported general vascular relaxation, including that in the renal vasculature, in very early pregnancy is also noticeable in the luteal phase of the menstrual cycle.

We measured in both luteal and follicular phase of the menstrual cycle, cardiac output, blood pressure, glomerular filtration rate, effective renal plasma flow and forearm blood flow. Measurements were performed in two consecutive menstrual cycles once on a low- and once on a high-salt diet. The data points obtained in the luteal phase were compared with those in the follicular phase of the same menstrual cycle. The follicular phases of the two menstrual cycles with exposure to two different salt-intakes were compared with each other. All comparisons were performed using non-parametric tests.

Only during high-salt menstrual cycles, we observed consistent changes with the menstrual cycle. Changing to a high-salt diet caused a decrease in plasma norepinephrine concentration and an increase in both glomerular filtration rate and filtration fraction. In the luteal phase total peripheral resistance was lower as compared to the follicular phase. As compared to the follicular phase, effective renal plasma flow and glomerular filtration rate were higher, and filtration fraction and renal vascular resistance lower in the luteal phase. Forearm blood flow and vascular resistance were comparable in the two phases of the menstrual cycle.

The results of this study provide evidence for a decrease in both total peripheral resistance and renal vascular resistance without consistent change in forearm vascular resistance in the luteal phase of the menstrual cycle. The glomerular filtration rate in the luteal phase has increased in concert with an increase in effective renal plasma flow and a decrease in filtration fraction. The hemodynamic changes induced by the endocrine environment of the luteal phase of the menstrual cycle mimic the hemodynamic adaptation to early pregnancy.

\section{INTRODUCTION}

The hemodynamic adaptation to pregnancy is characterized by the concomitant decrease in systemic and renal vascular resistances shortly after implantation and before blood volume (BV) expansion. Systemic arterial vasodilation, giving rise to a fall in filling pressure in the arterial vasculature, has been proposed as the primary trigger for the increase in BV during pregnancy ${ }^{108}$. The hemodynamic effects of this primary trigger are decreased systolic and diastolic blood pressures, increased cardiac output, stimulation of the renin-angiotensin-aldosterone axis, non-osmotic stimulation of thirst and vasopressin release, and renal sodium and water retention with expansion of the extracellular fluid volume (ECFV) and 
plasma volume (PV) compartments. In addition, renal arterial vasodilation leads to a $50-60 \%$ increase in renal blood flow (RBF) and a $40-50 \%$ increase in glomerular filtration rate (GFR). However, to the best of our knowledge, there is no other condition in mammalian physiology with, at the same time, expansion of the ECFV including PV and a markedly increased GFR. The mediator(s) of these very early (within three weeks of conception) circulatory adaptations is (are) still unknown.

Some of the hemodynamic changes seen in early pregnancy, although with a smaller amplitude, have been reported to take place in the luteal phase (LP) of the menstrual cycle. These changes consist of a fall in mean arterial pressure (MAP) ${ }^{7}$, a rise in GFR ${ }^{12,123}$ and plasma renin activity ${ }^{15}$, and a lowering of the osmotic thresholds for thirst and vasopressin release ${ }^{8}$. Most of these phenomena seem to represent compensations developing in response to peripheral arterial vasodilation resembling that in pregnancy. Recently, evidence for a combined decrease in systemic and renal vascular resistance in the LP of the menstrual cycle has been reported ${ }^{109}$. However, the sodium intake by the subjects in that study was not standardized throughout the menstrual cycle of measurement, an approach that may have influenced particularly the renal data.

The present study was designed to test the hypothesis that the reported general vascular relaxation, including that in the renal vasculature, in very early pregnancy is not only noticeable in the LP of the menstrual cycle, but also, that this effect is dependent upon sodium intake. To this end, we measured in both phases of two consecutive menstrual cycles with the subjects using a low-salt and a high-salt diet (or vice versa), respectively, cardiac output (CO), blood pressure (BP), GFR, effective renal plasma flow (ERPF) and forearm blood flow (FBF).

\section{MATERIALS AND METHODS}

\section{Subjects}

Experiments were performed in 29 healthy volunteers (mean age: $22 \pm 4$ (SD) years; mean body mass index: $\left.22 \pm 3(\mathrm{SD}) \mathrm{kg} / \mathrm{m}^{2}\right)$ with a history of regular menstrual cycles ( $29 \pm 3$ days). All participants gave written informed consent and the study was approved by the hospital's medical ethics committee. The investigation conforms with the principles outlined in the Declaration of Helsinki.

\section{Protocol}

A total of four measurements were performed divided over two consecutive menstrual cycles, in each cycle once in the follicular phase (FP), between the third - and seventh day, and once in the LP, between the 20th - and 24th day. During one cycle the subjects used a sodium-restricted diet of $50 \mathrm{mmol} /$ day and during the other 
a high-sodium diet of $220 \mathrm{mmol} /$ day. They started the diet seven days before each measurement. Each subject was randomized for type of diet to start with. Accordingly, half of the subjects started with the low-salt diet, and the other half with the high-salt diet. They collected a 24-hour urine sample for determination of sodium and creatinine contents the day before each measurement. Compliance with the diet was assessed by the 24-hour sodium excretion. Completeness of urine collection was verified by 24 -hour creatinine excretion. Measurements started at 8.00 a.m., after an overnight fast, and ended at 1.00 p.m.. The subjects were asked not to smoke and not to drink beverages containing caffeine or alcohol, and to drink water only, on the day of the measurements. Experimental conditions were similar on each of the four study days. Determinations of forearm blood flow and renal hemodynamics took place in a temperature-controlled room $\left(25-26^{\circ} \mathrm{C}\right)$.

All biophysical measurements were performed with the subject in supine position and the arm under investigation at heart level. Data acquisition was started after an acclimatization period of at least $30 \mathrm{~min}$. Before the acclimatization period, 20 -gauge catheters were inserted into a vein of both the right forearm for infusion of para-aminohippurate sodium (PAH) and inulin (see below) and the left forearm for blood sampling. At least $20 \mathrm{~min}$ after insertion of the catheter in the left forearm blood samples were drawn for measurement of progesterone, $17 \beta$-estradiol, catecholamines (norepinephrine, epinephrine and dopamine), $\mathrm{PAH}$ and inulin. Blood samples for measurement of catecholamines, $\mathrm{PAH}$ and inulin were centrifuged at $4^{\circ} \mathrm{C}$ for $10 \mathrm{~min}$ at $1500 \mathrm{~g}$, immediately after sampling, and stored deep-frozen before assay. The other blood samples were sent to the routine hospital laboratory for direct assay. Biophysical measurements included MAP, heart rate $(\mathrm{HR}), \mathrm{FBF}$ and various echocardiographic variables.

\section{Methods}

Both during the infusion of $\mathrm{PAH}$ and inulin and at the time of the echocardiographic measurements, BP and HR were measured in the left arm using a semiautomatic oscillometric device (Dinamap Vital Signs Monitor 1846, Critikon, Tampa, FL, USA).

FBF was determined in the left forearm using ECG-triggered strain-gauge venous occlusion plethysmography, a technique described in detail elsewhere ${ }^{88}$. In short, the cuff for venous occlusion was placed just above the elbow. A mercury straingauge was placed around the arm, $3-5 \mathrm{~cm}$ distal to the lateral humeral epicondyl. The hand circulation was stopped by inflating a wrist cuff to suprasystolic pressure starting one minute before each FBF measurement. Hence, FBF represents predominantly muscle blood flow ${ }^{89}$. FBF was measured over a period of four minutes. The mean of the last two minutes was used for calculations. A flow curve encompassed the time interval of five heart beats, during which venous occlusion 
$(50 \mathrm{mmHg})$ was applied over three heart beats. Thus, we measured on average 12 inflow curves $/ \mathrm{min}$. Forearm vascular resistance (FVR, in $\mathrm{mmHg} / \mathrm{ml} / \mathrm{dl} / \mathrm{min}$ ) was defined as the quotient of MAP and FBF.

Renal hemodynamics, i.e. ERPF and GFR, were assessed as the infusion clearance of PAH (PAH: aminohippurate sodium; MSD, West Point, PA, U.S.A.) and inulin (Inutest, Laevosan Gesellschaft, Linz, Austria), respectively. After the bolus injection, a continuous infusion was initiated using a syringe pump (Terumo SYC-521, Terumo Corporation, Tokyo, Japan) ${ }^{84}$. Blood was sampled, once before the bolus injection (blanks) and three times after an equilibration period of $120 \mathrm{~min}$, in heparinized glass tubes (Monoject, Sherwood Medical, St. Louis, $\mathrm{MO}$, U.S.). Blood samples were immediately centrifuged at $4{ }^{\circ} \mathrm{C}$ for $10 \mathrm{~min}$ at $1500 \mathrm{~g}$ and plasma stored deep-frozen prior to assay. Both $\mathrm{PAH}$ and inulin were measured as described elsewhere ${ }^{85,86}$. The infusion clearances of $\mathrm{PAH}$ and inulin were calculated by dividing the product of infusion rate and infusate concentration by the mean of plasma $\mathrm{PAH}$ and -inulin concentration after subtraction of the blanks, respectively. We calculated $\mathrm{RBF}$ and renal vascular resistance (RVR) using the formulas: $\mathrm{RBF}=\mathrm{ERPF} /(1$-hematocrit $)$ in $\mathrm{ml} / \mathrm{min}$ and $\mathrm{RVR}=$ (MAP/RBF) x 80,000 in dyn.s/ $\mathrm{cm}^{5}$. The filtration fraction (FF) was obtained by taking the ratio of the inulin clearance (GFR) and the ERPF. Renal fraction (RF) was calculated by taking the ratio of RBF and CO.

Echocardiographic measurements were performed in semi-left lateral position. The echocardiographic recordings were made with a cross-sectional, phased-array echocardiographic Doppler system (Hewlett-Packard Sonos 500 and 1000). This system utilizes a $2.5 \mathrm{MHz}$ phased array transducer and a $1.9 \mathrm{MHz}$ independent continuous-wave transducer. Data were registered on a strip chart recorder at a paper speed of $25 \mathrm{~mm} / \mathrm{s}$ for M-mode and at $50 \mathrm{~mm} / \mathrm{s}$ for Doppler measurements. $\mathrm{CO}$ was calculated according to the formula: $\mathrm{CO}=$ stroke volume $(\mathrm{SV}) \times \mathrm{HR}$. To determine SV, flow was measured across the aortic valve. Aortic velocities were recorded by means of the apical approach. A careful search for the maximal velocity was made. No angle correction was used to standardize measurements. The area under the velocity curve (aortic velocity integral) was recorded on disc. The mean aortic velocity integral was calculated from five consecutive ejections. The diameter of the aortic valve was measured off-line at the aortic orifice during systole with the M-mode echocardiographic aortic valve separation. SV was calculated as aortic area $\mathrm{x}$ aortic velocity integral. The aortic area was calculated as $1 / 4 \pi$ (aortic diameter) $)^{2}$. HR was derived from the averaged duration of five consecutive R-R intervals on the ECG. Total peripheral vascular resistance (TPR) was calculated by using the formula TPR $\left(\right.$ dyn.s $\left./ \mathrm{cm}^{5}\right)=\operatorname{MAP}(\mathrm{mmHg}) \times 80 / \mathrm{CO}(1 / \mathrm{min})$. In this formula, the MAP was calculated as the average of three recordings during measure- 
ment of the flow across the aortic valve using a semi-automatic oscillometric device (Dinamap Vital Signs Monitor 1846, Critikon, Tampa, FL, USA).

Serum $17 \beta$-estradiol and progesterone were determined using reagent kids of DPC (DPC, Los Angeles, Cal.). These assays are based on solid-phase radioimmunoassays using coated tube technology. Sensitivity of the assays are 0.035 and 0.15 $\mathrm{nmol} / \mathrm{l}$, respectively. For these two substances precision is better than $5 \%$. Norepinephrine (intra-assay CV 4.5\%, inter-assay CV 8.1\%), epinephrine (intra-assay CV $4.1 \%$, inter-assay CV 8.6\%) and dopamine (intra-assay CV 3.9\%, inter-assay CV $7.1 \%$ ) were assessed by a sensitive fluorimetric method, in which catecholamines are concentrated from plasma by liquid-liquid extraction and derivatized with a selective fluorescent agent prior to chromatography ${ }^{117}$. For each substance, all samples from the same individual were assayed in a single run.

\section{Statistical analysis}

Only ovulatory cycles were included for analysis of the differences between LP and FP, whereas all the cycles were included for analysis of differences between the FP's. A menstrual cycle was considered ovulatory when the mid-luteal serum progesterone concentration exceeded $15 \mathrm{nmol} / \mathrm{l}^{81}$.

Differences between LP and FP in each cycle (low and high-salt diet, $\mathrm{n}=12$ and 14 resp.), and in the FP between the high-salt - and low-salt diet cycles $(n=25)$ were analyzed by the Wilcoxon Matched-Pairs Signed-Ranks Test. Correlations between concomitantly measured variables or between differences in potentially related variables were tested by Spearman's rank correlation analysis. In the comparisons a p-value of less than $5 \%$ was considered statistically significant.

We calculated detectable changes in the mean of variables $(n=14, \alpha=0.05$ and $\beta$ $=0.10$ ), assuming a normal distribution of the data. The minimum detectable changes were $3.2 \%, 10.1 \%, 10.7 \%, 4.7 \%, 10.7 \%, 15.6 \%$ and $25.0 \%$ for the MAP, CO, TPR, GFR, ERPF, RVR and FBF, respectively.

\section{RESULTS}

Twenty-five of the 29 subjects completed all measurements (two menstrual cycles), whereas four subjects completed the two measurements in a single menstrual cycle (three low-salt menstrual cycles, one high-salt menstrual cycle). Four of the in total 54 menstrual cycles (one low-salt menstrual cycle, three high-salt menstrual cycles) were excluded from analysis because of irregular blood loss. Fourteen of the 23 (61\%) high-salt menstrual cycles and twelve of the 27 (44\%) low-salt menstrual cycles were ovulatory.

During the FP of high-salt menstrual cycles compared to the FP of low-salt menstrual cycles $(n=25)$ the GFR and FF was consistently higher by $6 \%$ and $9 \%$ (me- 
Table 1A. Hormonal characteristics and sodium excretion of subjects $(n=12)$ during the FP and LP of low-salt menstrual cycles.

\begin{tabular}{|c|c|c|c|}
\hline & FP & LP & Median difference \\
\hline $17 \beta$-estradiol $(\mathrm{nmol} / \mathrm{l})$ & $0.10(0.08-0.12)$ & $0.32(0.27-0.36)^{* *}$ & $+0.22(+0.14 \leftrightarrow+0.28)$ \\
\hline Progesterone (nmol/) & $2.1(1.3-2.3)$ & $25.3(20.4-45.0)^{* *}$ & $+22.7(+18.6 \leftrightarrow+47.3)$ \\
\hline Norepinephrine (nmol/l) & $0.63(0.54-1.10)$ & $0.65(0.61-0.79)$ & $+0.00(-0.19 \leftrightarrow+0.13)$ \\
\hline Epinephrine (nmol/) & $0.06(0.04-0.09)$ & $0.04(0.02-0.06)$ & $-0.02(-0.04 \leftrightarrow+0.00)$ \\
\hline Dopamine $(\mathrm{nmol} / \mathrm{l})$ & $0.13(0.08-0.19)$ & $0.14(0.08-0.17)$ & $+0.01(-0.08 \leftrightarrow+0.09)$ \\
\hline Sodium excr. (mmol/24h) & $56(38-66)$ & $46(34-86)$ & $+1.0(-27 \leftrightarrow+42)$ \\
\hline
\end{tabular}

Table 1B. Hormonal characteristics and sodium excretion of subjects $(n=14)$ during the FP and LP of high-salt menstrual cycles.

\begin{tabular}{|c|c|c|c|}
\hline & FP & LP & Median difference \\
\hline $17 \beta$-estradiol (nmol/l) & $0.10(0.06-0.12)$ & $0.36(0.26-0.45)^{* *}$ & $+0.28(+0.13 \leftrightarrow+0.35)$ \\
\hline Progesterone $(\mathrm{nmol} / \mathrm{I})$ & $1.6(1.2-2.6)$ & $31.2(27.5-36.0)^{\star *}$ & $+29.0(+24.4 \leftrightarrow+38.0)$ \\
\hline Norepinephrine (nmol/l) & $0.47(0.41-0.77)$ & $0.59(0.45-0.76)$ & $+0.06(-0.08 \leftrightarrow+0.26)$ \\
\hline Epinephrine (nmol/l) & $0.06(0.05-0.09)$ & $0.04(0.03-0.09)$ & $-0.02(-0.05 \leftrightarrow+0.02)$ \\
\hline Dopamine (nmol/l) & $0.13(0.09-0.22)$ & $0.14(0.07-0.24)$ & $-0.03(-0.06 \leftrightarrow+0.07)$ \\
\hline Sodium excr. (mmol/24h) & $220(172-279)$ & $200(156-244)$ & $-13(-101 \leftrightarrow+66)$ \\
\hline
\end{tabular}

dians), respectively, without concomitant change in ERPF, RVR and MAP. In addition, in the high-salt cycles plasma concentration of norepinephrine was $26 \%$ (median) lower than in the low-salt cycles.

Table $1 \mathrm{a}$ and $1 \mathrm{~b}$ list the hormonal status and urinary sodium excretion of the subjects during low-salt - $(\mathrm{n}=12)$ and high-salt diet $(\mathrm{n}=14)$, respectively. In the LP relative to the FP, serum $17 \beta$-estradiol and progesterone had increased by a comparable margin in the low-salt - and high-salt menstrual cycle. Neither catechola- 
Table 2A. Systemic and renal hemodynamics, and forearm blood flow during the FP and LP of low-salt menstrual cycles $(n=12)$.

\begin{tabular}{|c|c|c|c|}
\hline & FP & LP & Median difference \\
\hline $\operatorname{MAP}(\mathrm{mmHg})$ & $79(74-84)$ & $78(72-82)$ & $-3(-7 \leftrightarrow+3)$ \\
\hline Heart rate (beats/min) & $57(54-65)$ & $61(53-64)$ & $+1(-7 \leftrightarrow+7)$ \\
\hline Stroke volume $(\mathrm{m} / \mathrm{min})$ & $78.0(74.0-79.0)$ & $80.0(75.0-82.0)$ & $+1.0(-10.0 \leftrightarrow+4.0)$ \\
\hline Cardiac output (V/min) & $5.10(4.50-5.40)$ & $5.30(4.40-5.60)$ & $-0.05(-0.40 \leftrightarrow+0.90)$ \\
\hline TPR (dyn. $5 / \mathrm{cm}^{5}$ ) & $1338(1226-1424)$ & $1204(1067-1413)$ & $-60(-352 \leftrightarrow+171)$ \\
\hline GFR (mVmin) & $109(102-125)$ & $111(103-127)$ & $+5(-3 \leftrightarrow+8)$ \\
\hline $\operatorname{ERPF}(\mathrm{ml} / \mathrm{min})$ & $508(454-565)$ & $499(418-588)$ & $-9(-64 \leftrightarrow+46)$ \\
\hline Renal fraction (RBF/CO) & $0.16(0.15-0.18)$ & $0.16(0.13-0.18)$ & $+0.01(-0.03 \leftrightarrow+0.01)$ \\
\hline FF (GFRVERPF) & $0.21(0.20-0.25)$ & $0.23(0.20-0.25)$ & $+0.01(-0.01 \leftrightarrow+0.04)$ \\
\hline RVR (dyn.s/ $/ \mathrm{cm}^{5}$ ) & 7767 (7259-9279) & 8015 (7010-9615) & $-47(-984 \leftrightarrow+851)$ \\
\hline $\mathrm{FBF}(\mathrm{ml} / \mathrm{d} l / \mathrm{min})$ & $2.2(1.5-2.6)$ & $1.8(1.3-2.0)$ & $-0.4(-0.7 \leftrightarrow+0.4)$ \\
\hline $\mathrm{FVR}(\mathrm{mmH} \mathrm{g} / \mathrm{ml} / \mathrm{d} / / \mathrm{min})$ & $37.4(30.1-54.9)$ & $43.0(36.2-58.2)$ & $+5.8(-20 \leftrightarrow+18)$ \\
\hline \multicolumn{4}{|c|}{$\begin{array}{l}\text { Values during the FP and LP are medians and interquartile ranges. Median differences (wit) } \\
95 \% \text { confidence interval) are listed in the final column. Changes in variables during the men } \\
\text { strual cycle were evaluated using the Wilcoxon matched-pairs signed ranks test, }{ }^{\star} \mathrm{p}<0.05 \\
{ }^{\star *} \mathrm{p}<0.01 \text {. FP, follicular phase; LP, luteal phase; MAP, mean arterial pressure; TPR, total pe } \\
\text { ripheral vascular resistance; GFR, glomerular filtration rate; ERPF, effective renal plasma flow } \\
\mathrm{FF} \text {, filtration fraction; RVR, renal vascular resistance; FBF, forearm blood flow; FVR, forearn } \\
\text { vascular resistance. }\end{array}$} \\
\hline
\end{tabular}

mines nor sodium excretion differed between LP and FP in the two different salt cycles.

Table $2 \mathrm{a}$ and $2 \mathrm{~b}$ list the medians (interquartile ranges) and the median differences $(95 \% \mathrm{CI})$ for systemic and renal hemodynamics, and forearm blood flow during low-salt - $(\mathrm{n}=12)$ and high-salt diet $(\mathrm{n}=14)$, respectively. During the low-salt menstrual cycles none of the variables measured had changed consistently. During high-salt menstrual cycles HR was higher, TPR was lower and the MAP showed a tendency to be lower $(p=0.06)$ in the LP. Cyclic changes in renal hemodynamics consisted of a higher GFR and ERPF, and a lower RVR and FF. Finally, the RF tended to be higher in the LP relative to the FP ( $p=0.07)$.

In both low- and high-salt menstrual cycles, changes in TPR did neither correlate with changes in serum progesterone concentration nor with the serum $17 \beta$-estradiol concentration or the ratio of serum progesterone to serum $17 \beta$-estradiol. 
Table 2B. Systemic and renal hemodynamics, and forearm blood flow during the FP and LP of high-salt menstrual cycles $(n=14)$.

\begin{tabular}{|c|c|c|c|}
\hline & FP & LP & Median difference \\
\hline $\mathrm{MAP}(\mathrm{mmHg})$ & $82(77-85)$ & $78(75-83)$ & $-1(-4 \leftrightarrow+1)$ \\
\hline Heart rate (beats/min) & $59(55-61)$ & $62(56-67)^{*}$ & $+3(+1 \leftrightarrow+7)$ \\
\hline Stroke volume ( $\mathrm{ml} / \mathrm{min})$ & $76.0(69.0-84.5)$ & $78.0(68.5-81.3)$ & $-0.5(-4.0 \leftrightarrow+5.0)$ \\
\hline Cardiac output (V/min) & $4.70(4.50-5.25)$ & $4.85(4.40-5.28)$ & $-0.05(-0.5 \leftrightarrow+0.6)$ \\
\hline TPR (dyn. $\left.s / \mathrm{cm}^{5}\right)$ & $1336(1226-1453)$ & $1320(1178-1441)^{\star}$ & $-71(-179 \leftrightarrow-10)$ \\
\hline $\mathrm{GFR}(\mathrm{ml} / \mathrm{min})$ & $117(109-127)$ & $123(104-137)^{\star}$ & $+4(+1 \leftrightarrow+13)$ \\
\hline $\operatorname{EFPF}(\mathrm{ml} / \mathrm{min})$ & $492(401-583)$ & $549(475-647)^{* *}$ & $+43(+10 \leftrightarrow+116)$ \\
\hline Renal fraction (RBF/CO) & $0.16(0.13-0.19)$ & $0.18(0.15-0.20)$ & $+0.01(-0.01 \leftrightarrow+0.04)$ \\
\hline FF (GFR/ERPF) & $0.24(0.21-0.28)$ & $0.22(0.21-0.24)^{\star}$ & $-0.02(-0.05 \leftrightarrow-0.01)$ \\
\hline $\operatorname{RVR}\left(\right.$ dyn. $s / \mathrm{cm}^{5}$ ) & 8373 (7236-9885) & $7562(6352-8515)^{*}$ & $-563(-2613 \leftrightarrow-50)$ \\
\hline $\mathrm{FBF}(\mathrm{mV} / \mathrm{d} / \mathrm{min})$ & $2.1(1.4-2.5)$ & $2.0(1.7-2.6)$ & $+0.1(-0.4 \leftrightarrow+0.9)$ \\
\hline $\mathrm{FVR}(\mathrm{mmHg} / \mathrm{ml} / \mathrm{dl} / \mathrm{min})$ & $40.1(31.2-57.3)$ & $39.3(30.4-45.2)$ & $-0.2(-14 \leftrightarrow+8.9)$ \\
\hline $\begin{array}{l}\text { Values during the FP an } \\
95 \% \text { confidence interva } \\
\text { strual cycle were evalua } \\
{ }^{\star \star} \mathrm{p}<0.01 \text {. FP, follicula } \\
\text { ripheral vascular resistan } \\
\mathrm{FF} \text {, filtration fraction; RI } \\
\text { vascular resistance. }\end{array}$ & $\begin{array}{l}\text { d LP are medians an } \\
\text { l) are listed in the fin } \\
\text { ted using the Wilcox } \\
\text { r phase; LP, luteal ph } \\
\text { nce; GFR, glomerular } \\
\text { VR, renal vascular res }\end{array}$ & $\begin{array}{l}\text { d interquartile ranges } \\
\text { al column. Changes in } \\
\text { con matched-pairs sig } \\
\text { lase; MAP, mean arter } \\
\text { filtration rate; ERPF, e } \\
\text { sistance; FBF, forearm }\end{array}$ & $\begin{array}{l}\text { s. Median differences (witl } \\
n \text { variables during the men } \\
\text { gned ranks test, }{ }^{\star} p<0.05 \\
\text { rial pressure; TPR, total pe } \\
\text { effective renal plasma flow } \\
\text { blood flow; FVR, forearm }\end{array}$ \\
\hline
\end{tabular}

In neither low- nor high-salt menstrual cycles, the change in GFR correlated with the change in serum progesterone -, serum 17 $\beta$-estradiol concentration, ERPF, RVR or the ratio of serum progesterone to serum $17 \beta$-estradiol. Also, we could not demonstrate a correlation between changes in ERPF, RVR, RF on the one hand and changes in serum progesterone -, serum 17 $\beta$-estradiol concentration, or the ratio of serum progesterone to serum $17 \beta$-estradiol on the other hand. Neither during low- nor during high-salt menstrual cycles, did changes in FF correlate with changes in MAP. Finally, irrespective of the diet, the change in RBF did not correlate with the change in $\mathrm{CO}$. 
The objective of this study was to determine whether the general vascular relaxation, and renal vascular relaxation in particular, as they develop in the first weeks of pregnancy, are also noticeable in the LP of the menstrual cycle. Our data supported this concept only partly. We found a decrease in TPR in the LP relative to the FP. This decrease in TPR may not be evenly distributed over the systemic circulation as a consistent fall in RVR was paralleled by inconsistent changes in FVR. Interestingly this effect was only observed in subjects adhered to a high-salt diet. The hormonal cyclicity in our study was expressed by a $0.22-0.28 \mathrm{nmol} / \mathrm{l}$ higher serum $17 \beta$-estradiol in combination with a $22.7-29.0 \mathrm{nmol} / \mathrm{l}$ higher serum progesterone in the LP as compared to the FP. The latter can be expected to have induced indirectly, through the well-known $0.3-0.5^{\circ} \mathrm{C}$ increase in core temperature, the higher HR and lower TPR in the LP. Animal studies ${ }^{78}$ and in-vitro studies using human placental arteries and veins ${ }^{79}$ have shown that both progesterone ${ }^{78,79}$ as well as $17 \beta$-estradiol ${ }^{80}$ have vasodilating properties. To what extent these two steroids act in concert in the LP of the menstrual cycle, to induce the observed decrease in RVR and TPR is still obscure. We were unable to demonstrate a correlation between changes in hormonal environment and changes in TPR, RVR and RF. This may be related to the fact that vasorelaxation like the regulation of core temperature, is not dose-dependent. The vaso-relaxant effect of the steroid hormones was not discernible when the subjects used a salt-restricted diet. Switching to a low-salt diet led to an increase in plasma norepinephrine. It is tempting to speculate that this causes an increased vascular tone, possibly, mediated by a decreased vascular filling state. In this concept, the cyclicity in renal hemodynamics and TPR may have been overruled by this higher vascular tone. Possibly, optimum hydration is a prerequisite for measurable vascular relaxation in the LP of the menstrual cycle.

TPR was calculated by simultaneous non-invasive measurement of CO by Doppler echocardiography and MAP by using an oscillometric device. Continuouswave Doppler measurement of flow in the aortic valve has been found to provide a reliable and reproducible estimate for stroke volume ${ }^{118,119}$. Kusumoto et al ${ }^{120}$ demonstrated that continuous-wave Doppler provides a reliable estimate of $\mathrm{CO}$ in the study of serial changes in $\mathrm{CO}$ in response to various interventions. In the present study CO had not changed by more than $10 \%$ within the menstrual cycle. This observation agrees with the results reported by others using different methods ${ }^{121,124}$. Only Chapman et al. ${ }^{109}$ demonstrated a $18 \%$ higher CO in the LP compared to the FP using a comparable study protocol and methodology. It is possible that a higher salt intake in the LP of the menstrual cycle as compared to the FP, may have magnified the increase in $\mathrm{CO}$ in the latter study, e.g. by a larger increase in ECFV and with it, preload. The relation between TPR and the men- 
strual cycle has also been studied previously ${ }^{105,109,121}$. By impedance cardiography, it was demonstrated that the vascular tone in the LP was lower than in the $\mathrm{FP}^{105}$. However, this effect was only seen in response to stress. Lehtovirta et al. ${ }^{121}$ did not find cyclicity in TPR during the menstrual cycle. However, their study group ( $n=8)$ was too small to generate sufficient power to discern small changes in TPR. They also compared the late FP with the LP, an approach that can be expected to reduce the differences in e.g. serum $17 \beta$-estradiol concentration. Chapman et al. ${ }^{109}$ reported of a much greater cyclic effect on the TPR in the LP: $22 \%$ lower compared to the FP, obviously, as a direct consequence of a much larger $\mathrm{CO}$ in the LP of the menstrual cycle. The fact that the majority of women were initially studied in the FP of the menstrual cycle might have increased their observed change in TPR. Because both 17 $\beta$-estradiol and progesterone can be held responsible, at least in animal studies and in-vitro ${ }^{78,79}$, for the observed decrease in TPR, we expected to find a correlation between the change in serum $17 \beta$-estradiol concentration, that in progesterone concentration and that in the ratio of serum progesterone to $17 \beta$-estradiol in the LP on the one hand, and the change in TPR on the other hand. The fact, that we were unable to find such correlations indicates that the hemodynamic effects of fluctuations in endocrine environment during the menstrual cycle do not follow a simple dose-response relationship.

To our knowledge, in only three studies the GFR and ERPF were studied together in their relation with the menstrual cycle ${ }^{11,12,109}$. In two studies ${ }^{11,12}$ a trend towards an increased ERPF was noticed without a consistent change in FF, but the changes were small and did not reach statistical significance. The latter was at least in part caused by the relatively high variability of the methodology employed. Only Chapman et al. ${ }^{109}$ demonstrated a comparable increase in GFR and ERPF, and thus without change in the FF, in the LP compared to the FP. In our study the ERPF seemed to have increased by a larger fraction than the GFR thus giving rise to a consistent fall in FF in the LP. It is possible that the salt-intake in the study by Chapman and coworkers was higher in the LP relative to the FP, causing e.g. a larger luteal GFR increase. On the other hand, the absolute differences are so small that they may have been a result of random error and/or slightly different methodology. To reduce the variability in the measurements, induced by differences in salt intake, we asked the subjects to adhere strictly to their salt diet. Due to a larger percentage increase in ERPF than in GFR in the LP compared to the FP, we observed a modest fall in FF. In this study neither the changes in GFR and ERPF were correlated with each other during the menstrual cycle, nor did these changes correlate with the change in sex steroids. So, it is not certain whether the GFR increase in the LP is directly related to the observed increase in ERPF. Furthermore, these data do not provide insight in the mechanism that leads to these renovascular changes in the menstrual cycle. The concomitantly lower FF in the LP, suggests 
that the relaxation stimulus has a stronger dilatating effect on the efferent - than on the afferent renal arterioles.

If systemic vascular relaxation in the LP would be evenly distributed over the vascular bed, one would also expect to find a higher blood flow in muscle tissue. We did neither find a higher FBF nor a lower FVR in the LP. Although, the minimum detectable change was large in our study $(25 \%)$, similar observations have been reported by others ${ }^{12,93-96}$. Therefore, we feel that the latter result together with the other data in this study, provide evidence for a selective vascular relaxation in the LP of the menstrual cycle. The observed tendency of the RF to increase during high-salt menstrual cycles supports this concept. The stimulus for such a selective vaso-relaxation remains to be identified.

In conclusion, the present data show, in case of sufficient salt intake, a decrease in both TPR and RVR in combination with inconsistent changes in FVR in the LP of the menstrual cycle. The increase in GFR in the LP is paralleled by an increase in ERPF and a decrease in FF. Post-ovulatory vasodilation of efferent, more than afferent, renal arterioles could be responsible for the raised GFR. The hemodynamic changes induced by the endocrine environment of the LP of the menstrual cycle are modified by salt intake, and mimic the hemodynamic adaptation to early pregnancy.

\section{ACKNOWLEDGMENTS}

We thank Hubert J. A. Schouten for his valuable contributions to the statistical analysis. 


\section{The contribution of endogenous progesterone and $17 \beta$-estradiol to the hemodynamic and neurohumoral changes during the menstrual cycle}

E. van Beek, A.J.H.M. Houben*, T.H.A. Ekhart*, P.M.H. Schiffers***,

E.C. Cheriex**, P.W. de Leeuw*, L.L.H. Peeters

Departments of Obstetrics and Gynecology, Internal Medicine*, and Cardiology**, University Hospital Maastricht and department of Pharmacology***, University of Maastricht, Maastricht, The Netherlands.

Submitted 


\section{ABSTRACT}

This study was designed to test the hypothesis that endogenous progesterone is needed for the development of the hemodynamic changes in the luteal phase of the menstrual cycle.

We measured in both luteal and follicular phase of the menstrual cycle, cardiac output, blood pressure, glomerular filtration rate, effective renal plasma flow, forearm blood flow, plasma volume and their regulatory neurohormones (reninangiotensin-aldosterone system and ANP). Measurements were performed while the subjects used a standardized high-salt diet. A menstrual cycle was considered ovulatory when the mid-luteal serum progesterone concentration exceeded 15 $\mathrm{nmol} / \mathrm{l}$ and anovulatory when it was less than $15 \mathrm{nmol} / \mathrm{l}$. Differences between luteal phase and follicular phase in the menstrual cycle, as well as differences in cyclic changes and baseline values (follicular phase) between groups (ovulatory and anovulatory) were analyzed using non-parametric tests.

Under basal condition (follicular phase), only, serum 17ß-estradiol level was higher in the ovulatory group as compared to that in the anovulatory group. The ovulatory group differed from the anovulatory group by a rise in the luteal phase relative to the follicular phase, in effective renal plasma flow and renal fraction, and a fall in filtration fraction. Cyclic changes in systemic vascular resistance, glomerular filtration rate, plasma volume and neurohormones were comparable in both groups.

The results of this study suggest that the luteal fall in systemic vascular resistance and the rise in glomerular filtration rate are primarily $17 \beta$-estradiol-mediated, whereas, the luteal rise in effective renal plasma flow is a selective progesterone effect. Possibly, progesterone down-regulates the angiotensin AT1 receptor in the efferent arteriole of the glomerulus.

\section{INTRODUCTION}

In the luteal phase of the menstrual cycle (LP), renal and systemic vascular resistance fall, while sodium and fluid retaining mechanisms are activated ${ }^{125}$. When ovulation is followed by conception and implantation, these luteal changes become more pronounced in the following weeks of pregnancy ${ }^{126}$. Neither the trigger for the changes in the LP, nor the one giving rise to their post-implantation amplification, have been identified. The LP differs from the follicular phase (FP) by higher levels of progesterone and $17 \beta$-estradiol, steroids with known vasodilating properties ${ }^{78-80}$. Whether these two steroids, alone or in combination, are directly responsible for the changes in hemodynamics during the LP, is unknown. It is conceivable that the role of these two steroids is limited to the institution of a 
"luteal" endocrine environment, necessary for some other not (yet) identified vasodilator substance to become activated. Disclosure of the mechanism responsible for the luteal changes can be expected to improve our insight in the subsequent superimposed changes in early pregnancy.

The objective of the present study was to test the hypothesis that endogenous progesterone is needed for the development of the hemodynamic changes in the LP of the menstrual cycle. To this end, we compared cyclic changes in systemic vascular resistance, renal hemodynamics and volume homeostasis in anovulatory cycles with those observed in ovulatory cycles. In a previous study we noted, that cyclic changes in renal hemodynamics are largest when subjects are using a high-salt intake ${ }^{127}$. Therefore, we performed our measurements while the subjects used a standardized high-salt diet.

\section{MATERIALS AND METHODS}

\section{Subjects}

Experiments were performed in 29 healthy volunteers (mean age: $22 \pm 4$ (SD) years; mean body mass index: $22 \pm 3(\mathrm{SD}) \mathrm{kg} / \mathrm{m}^{2}$ ) with a history of regular menstrual cycles ( $29 \pm 3$ days). All participants gave written informed consent and the study was approved by the hospital's medical ethics committee. The investigation conformed with the principles outlined in the Declaration of Helsinki.

\section{Protocol}

Two measurements were performed in the menstrual cycle, once in the follicular phase (FP), between the third - and seventh day, and once in the LP, between the 20 th - and 24th day. The subjects used a high-sodium diet of $220 \mathrm{mmol} /$ day. They started the diet seven days before each measurement. They collected a 24hour urine sample for determination of sodium and creatinine contents the day before each measurement. Compliance with the diet was assessed by the 24-hour sodium excretion. Completeness of urine collections was verified by 24 -hour creatinine excretions. Measurements started at 8.00 a.m., after an overnight fast, and ended at 1.00 p.m.. The subjects were asked not to smoke and not to drink beverages containing caffeine or alcohol, and to drink water only, on the day of the measurements. Experimental conditions were similar on both study days. All measurements were performed with the subject in supine position and the arm under investigation at heart level, after an acclimatization period of at least $30 \mathrm{~min}$. Forearm blood flow (FBF) and renal hemodynamics were measured in a temperature-controlled room $\left(25-26^{\circ} \mathrm{C}\right)$. Before the acclimatization period, a 20 -gauge catheter was inserted into a vein of the right forearm for infusion of para-aminohippurate sodium (PAH) and inulin (see renal hemodynamics). An- 
other 20-gauge catheter was inserted into a vein of the left forearm in order to take blood samples. At least $20 \mathrm{~min}$ after insertion of the catheter blood samples were drawn from the left catheter for measurement of hematocrit, osmolality, albumin, progesterone, $17 \beta$-estradiol, active plasma renin concentration (APRC), angiotensin II (AngII), aldosterone (Aldo), atrial natriuretic peptide (ANP), PAH and inulin.. Blood samples for measurement of APRC, AngII, Aldo, ANP, PAH, and inulin were centrifuged at $4^{\circ} \mathrm{C}$ for $10 \mathrm{~min}$ at $1500 \mathrm{~g}$, immediately after sampling, and stored deep-frozen before assay. The other blood samples were sent to the hospital laboratories for direct determination. Besides sampling, mean arterial pressure (MAP), heart rate (HR), FBF were determined, followed by echocardiography and measurement of plasma volume (PV).

\section{Measurements}

During the infusion of $\mathrm{PAH}$ and inulin, and at the time of echocardiographic measurements, blood pressure and HR were measured in the left arm using a semi-automatic oscillometric device (Dinamap Vital Signs Monitor 1846, Critikon, Tampa, FL, USA).

FBF was determined in the left forearm using ECG-triggered strain gauge venous occlusion plethysmography, a technique described in detail elsewhere ${ }^{88}$. In short, the cuff for venous occlusion was placed just above the elbow. A mercury strain gauge was placed around the arm, $3-5 \mathrm{~cm}$ distal to the lateral humeral epicondyl. The hand circulation was stopped by inflating a wrist cuff to suprasystolic pressure starting one minute before each FBF measurement. Hence, FBF represents predominantly muscle blood flow ${ }^{89}$. FBF was measured over a period of four minutes. The mean of the last two minutes was used for calculations. A flow curve encompassed the time interval of five heart beats, during which venous occlusion $(50 \mathrm{mmHg})$ was applied over three heart beats. Thus, we measured on average 12 inflow curves $/ \mathrm{min}$. Forearm vascular resistance (FVR, in $\mathrm{mmHg} / \mathrm{ml} / \mathrm{dl} / \mathrm{min}$ ) was calculated by using the formula: MAP/FBF.

Renal hemodynamics, i.e. effective renal plasma flow (ERPF) and glomerular filtration rate (GFR), were assessed from the clearance of PAH (PAH: aminohippurate sodium; MSD, West Point, PA, U.S.A.; coefficient of variation (CV) 8\%) and inulin (Inutest, Laevosan Gesellschaft, Linz, Austria; CV 6\%), respectively, using the constant infusion method. After the bolus injection, a continuous infusion was started using a syringe pump (Terumo SYC-521, Terumo Corporation, Tokyo, Japan) ${ }^{84}$. Blood was sampled in heparinized glass tubes (Monoject, Sherwood Medical, St. Louis, MO, U.S.) once before the bolus injection (blanks) and, after an equilibration period of $120 \mathrm{~min}$, thrice at ten min intervals. Blood was centrifuged at $4{ }^{\circ} \mathrm{C}$ for $10 \mathrm{~min}$ at $1500 \mathrm{~g}$ directly after sampling. All plasma sam- 
ples were stored deep-frozen prior to assay. PAH and inulin were measured as detailed elsewhere ${ }^{85,86}$. The infusion clearances of $\mathrm{PAH}$ and inulin were calculated by dividing the product of infusion rate and infusate concentration by the mean of the three plasma PAH and - inulin concentrations, after subtraction of the blanks, respectively. We calculated renal blood flow $(\mathrm{RBF}$, in $\mathrm{ml} / \mathrm{min}$ ) and renal vascular resistance (RVR, in dyn.s $\left./ \mathrm{cm}^{5}\right)$ using the formulas: ERPF/(1-Hematocrit) and (MAP/RBF) 880000 , respectively. The filtration fraction (FF) was obtained by taking the ratio of the GFR and ERPF. Renal fraction (RF) was calculated by taking the ratio of RBF and cardiac output (see echocardiographic measurements). All data were standardized for body-surface area.

Echocardiographic measurements, as described in detail elsewhere ${ }^{112}$, were performed in semi-left lateral position after approximately five minutes of rest. The echocardiographic recordings were made with a cross-sectional, phased array echocardiographic Doppler system (Hewlett-Packard Sonos 500 and 1000). Cardiac output (CO) was calculated according to the formula: $\mathrm{CO}=$ stroke volume (SV) $x$ heart rate (HR). To determine SV, flow was measured across the aortic valve. SV was calculated as aortic area $\mathrm{x}$ aortic velocity integral. Total peripheral vascular resistance $($ TPR $)$ was calculated by using the formula TPR $\left(\right.$ dyn.s $\left./ \mathrm{cm}^{5}\right)=$ $\mathrm{MAP}(\mathrm{mmHg}) \times 80 / \mathrm{CO}(1 / \mathrm{min})$. In this formula, the MAP was calculated as the average of three recordings during measurement of the flow across the aortic valve using the semi-automatic oscillometric device. All data were standardized for body-surface area.

Plasma volume (PV, CV 4.2\%) was measured, at approximately the same time in the morning, as follows ${ }^{110}$. Five $\mu \mathrm{Ci}{ }^{125} \mathrm{I}$-human albumin, dissolved in $1 \mathrm{ml} \mathrm{sa-}$ line, was given intravenously at time zero (total body dose of irradiation $=0.04$ $\mathrm{mSv}$ ). Before the bolus injection and again after 10,20 and $30 \mathrm{~min}$, blood samples $(5 \mathrm{ml})$ were drawn to determine the volume specific plasma radioactivity $(\mathrm{mCi} / \mathrm{ml})$. The virtual volume specific plasma radioactivity at time zero was calculated by extrapolation using the three serially obtained volume specific plasma radioactivity data points after bolus injection. PV was obtained by dividing the total injected radioactivity by the virtual volume specific plasma radioactivity at time zero, corrected for blanks. To eliminate the impact of body fat on PV per kg body weight, we calculated the body fat fraction ${ }^{128}$, in order to be able to express the $\mathrm{PV}$ in $\mathrm{ml} / \mathrm{kg}$ lean body mass.

Serum $17 \beta$-estradiol and progesterone were determined using reagent kids of DPC (DPC, Los Angeles, Cal.). These assays are based on solid-phase radioimmunoassays using coated tube technology. Sensitivity of the assays are 0.035 and 0.15 nmol/l, respectively. 
APRC was assessed by the IRMA-method (Nichols Institute Diagnostics, Wychen, The Netherlands) with an intra-assay coefficient of variation (CV) of $2.9 \%$ and an inter-assay $\mathrm{CV}$ of $7.6 \%{ }^{113}$. AngII was determined by radio-immunoassay (RIA) following Ph Phenyl column extraction (Amersham Int. Amersham, UK; intra-assay CV $4.6 \%$ and inter-assay CV 7.7\%) ${ }^{114}$. Aldo was assayed by means of a solid-phase protein binding RIA (Diagnostic Products Corporation, LA, USA; intra- and inter assay CV $4.3 \%$ and $6.7 \%$ resp. ${ }^{115}$. ANP was measured by a competitive protein-binding radio-immunoassay (Nichols Institute Diagnostics; intra- and inter assay CV $7.0 \%$ and $12.9 \%$, resp) following extraction of plasma over Sep-Pak C18 columns ${ }^{116}$. For each hormone, all samples from the same individual were assayed in a single run.

Statistical analysis

A menstrual cycle was considered ovulatory when the mid-luteal serum progesterone concentration exceeded $15 \mathrm{nmol} / \mathrm{l}^{81}$ and anovulatory when it was less than 15 $\mathrm{nmol} / \mathrm{l}$.

Differences in cyclic changes and baseline values (FP) between the ovulatory - and anovulatory group were analyzed by the Mann-Whitney-U Test. Differences between LP and FP in the menstrual cycle were analyzed by the Wilcoxon MatchedPairs Signed-Ranks Test. Correlations between potentially related variables were tested by Spearman's rank correlation analysis. In the comparisons a p-value of less than $5 \%$ was considered statistically significant.

\section{RESULTS}

Twenty-six subjects completed the two measurements in a high-salt menstrual cycle. Four of these were excluded from analysis because of irregular blood loss. Fourteen of the menstrual cycles were ovulatory, whereas eight were anovulatory. Before testing differences in cyclic changes between ovulatory and anovulatory subjects, we evaluated the inter-group differences for all variables under basal conditions (FP). Only serum $17 \beta$-estradiol level was higher $(p=0.02)$ in the FP of the ovulatory group as compared to that in the anovulatory group.

Table 1 lists the median values in the FP and the median differences (with 95\% confidence intervals) between both phases of the menstrual cycle, for sex steroids and urinary sodium excretion in the ovulatory - and anovulatory group. Only the change in serum progesterone was significantly greater (Fig. 1a, p $<0.001$ ) in the ovulatory group compared to the anovulatory group.

Medians of cyclic changes (with 95\% CI) for central hemodynamics, renal variables and forearm blood flow in both groups are listed in Table 2. The ovulatory 
Table 1. Hormonal characteristics and sodium excretion of subjects during ovulatory and anovulatory menstrual cycles.

\begin{tabular}{|c|c|c|c|c|c|}
\hline & \multicolumn{2}{|c|}{ Ovulatory cycle $(n=14)$} & \multicolumn{3}{|c|}{ Anovulatory cycle $(n=8)$} \\
\hline & $\mathrm{FP}^{\dagger}$ & $\mathrm{LP}-\mathrm{FP}{ }^{\ddagger}$ & $\mathrm{FP}^{\dagger}$ & $\mathrm{LP}-\mathrm{FP}^{\ddagger}$ & p-value ${ }^{\S}$ \\
\hline $\begin{array}{l}\text { 17ß-estradiol } \\
(\mathrm{nmol} / /)\end{array}$ & $\begin{array}{l}0.10 \\
(0.06-0.12)\end{array}$ & $\begin{array}{l}+0.28 * * \\
(+0.13 \leftrightarrow+0.35)\end{array}$ & $\begin{array}{l}0.07 \\
(0.06-0.07)\end{array}$ & $\begin{array}{l}+0.21 * \\
(+0.04 \leftrightarrow+0.31)\end{array}$ & $p=0.14$ \\
\hline $\begin{array}{l}\text { Progesterone } \\
(\mathrm{nmo} / /)\end{array}$ & $\begin{array}{l}1.6 \\
(1.2-2.6)\end{array}$ & $\begin{array}{l}+29.0^{\star *} \\
(+24.4 \leftrightarrow+38.0)\end{array}$ & $\begin{array}{l}1.9 \\
(1.5-2.0)\end{array}$ & $\begin{array}{l}+4.8^{\star} \\
(+2.9 \leftrightarrow+9.0)\end{array}$ & $p<0.001$ \\
\hline $\begin{array}{l}\text { Sodium excr. } \\
\text { (mmol/24h) }\end{array}$ & $\begin{array}{l}220 \\
(172-279)\end{array}$ & $\begin{array}{l}-13 \\
(-101 \leftrightarrow+66)\end{array}$ & $\begin{array}{l}192 \\
(148-234)\end{array}$ & $\begin{array}{l}-32 \\
(-118 \leftrightarrow+44)\end{array}$ & $p=0.71$ \\
\hline
\end{tabular}

Medians and interquartile ranges of the $\mathrm{FP}^{\dagger}$, and median differences (with $95 \%$ confidence interval) between $\mathrm{LP}$ and $\mathrm{FP}{ }^{\ddagger}$ are listed. Changes in variables during the menstrual cycle were evaluated using the Wilcoxon matched-pairs signed ranks test $\left({ }^{*} p<0.05,{ }^{* *} p<0.01\right)$. Differences in cyclic changes between ovulatory and anovulatory cycles were analyzed by the Mann-Whitney-U test ${ }^{5}$. FP, follicular phase; LP, luteal phase; sodium excr., sodium excretion.

group differed from the anovulatory group by a rise in ERPF and RF, and a fall in FF in the LP.

Cyclic changes in PV and neurohormones (Table 3) were comparable in both the ovulatory - and anovulatory group. Most relevant variables are also shown in Figs. 2 and 3.

Neither in the ovulatory, nor in the anovulatory as well as in the total group, changes in serum progesterone and $17 \beta$-estradiol concentration correlated with concomitant changes in any of the measured hemodynamic or neurohumoral variables. 

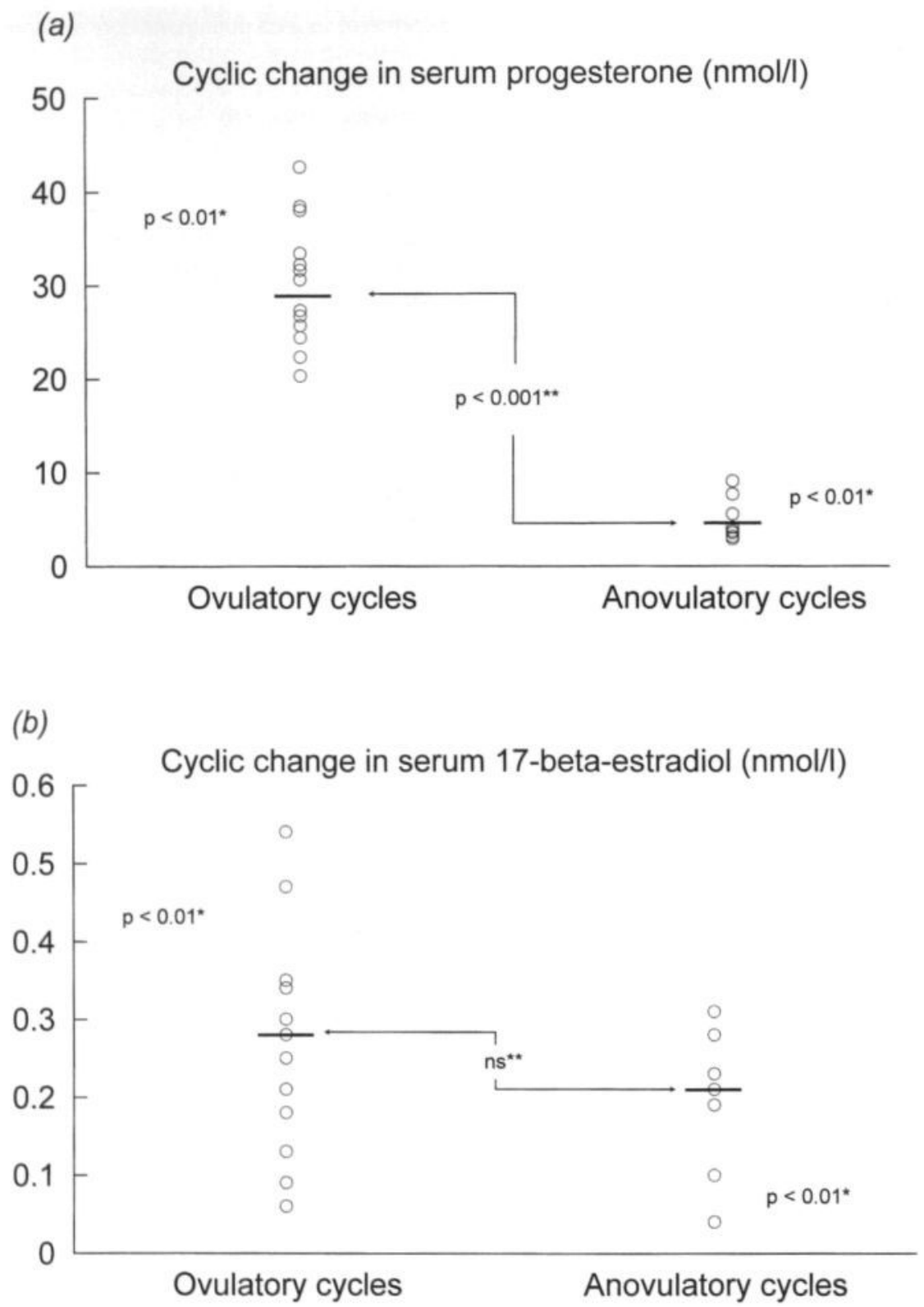

Figure 1. Changes during ovulatory and anovulatory cycles in serum progesterone ( $\mathrm{nmo} / /$ ) (a) and serum 17- $\beta$-estradiol (nmol/l) (b). * Wilcoxon Matched-Pairs Signed Ranks Test, ** MannWhitney-U Test. 
Table 2. Central hemodynamic variables, renal hemodynamics and forearm blood flow of subjects during ovulatory and anovulatory menstrual cycles.

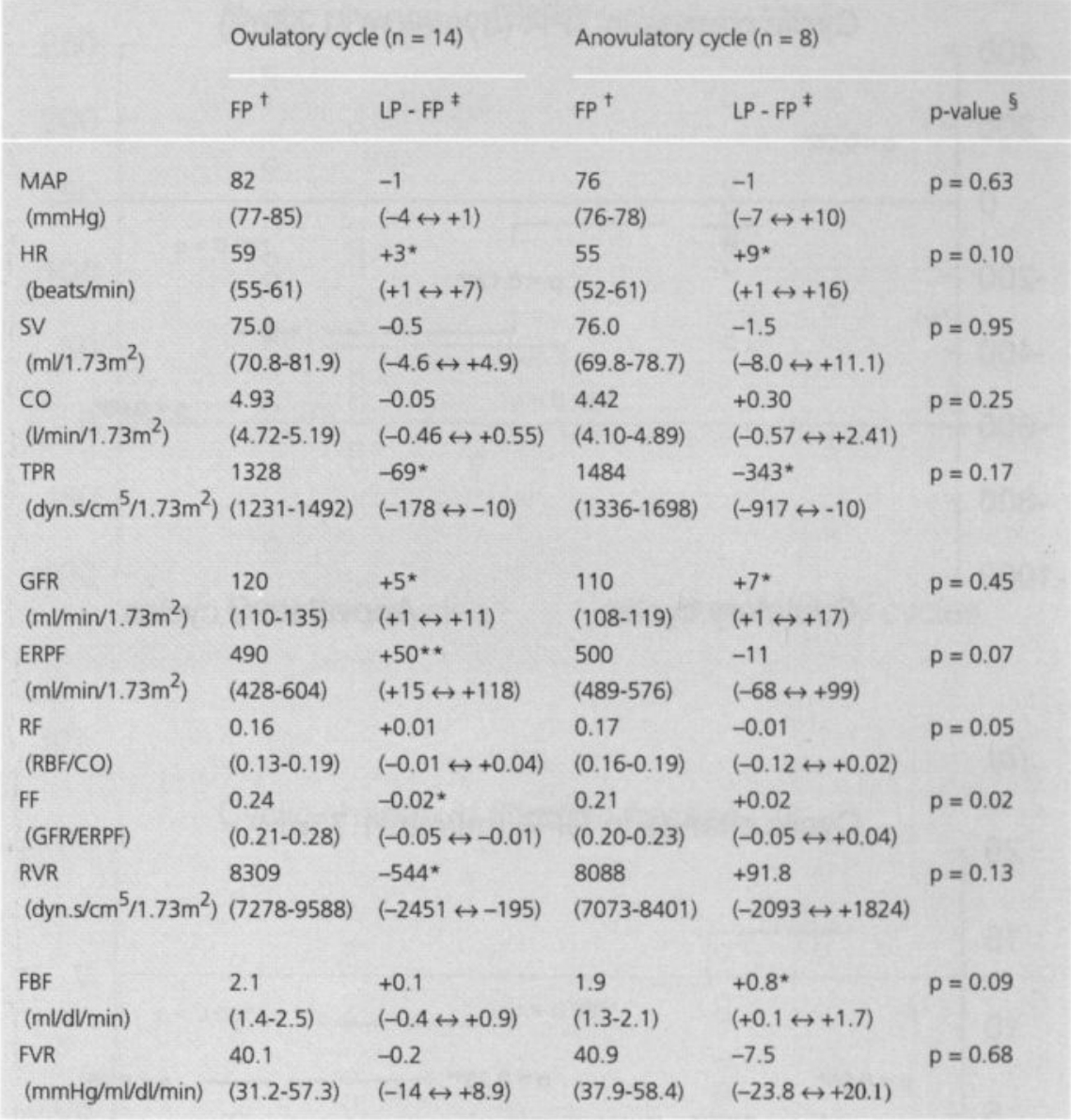

Medians and interquartile ranges of the $\mathrm{FP}^{\dagger}$, and median differences (with $95 \%$ confidence interval) between LP and FP ${ }^{\ddagger}$ are listed. Changes in variables during the menstrual cycle were evaluated using the Wilcoxon matched-pairs signed ranks test $\left({ }^{\star} p<0.05,{ }^{* \star} p<0.01\right)$. Differences in cyclic changes between ovulatory and anovulatory cycles were analyzed by the Mann-Whitney-U test ${ }^{\mathfrak{5}}$. FP, follicular phase; LP, luteal phase; MAP, mean arterial pressure; HR, heart rate; SV, stroke volume; CO, cardiac output; TPR, total peripheral vascular resistance; GFR, glomerular filtration rate; ERPF, effective renal plasma flow; RF, renal fraction; FF, filtration fraction; RVR, renal vascular resistance; FBF, forearm blood flow; FVR, forearm vascular resistance. 
(a)

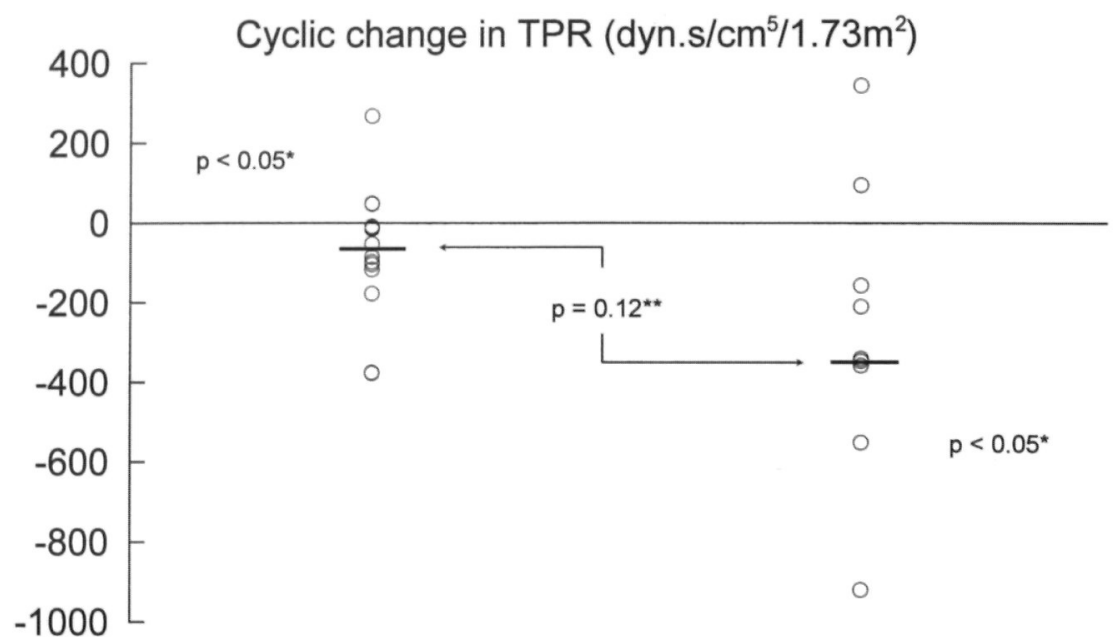

Ovulatory cycles

Anovulatory cycles

(b)

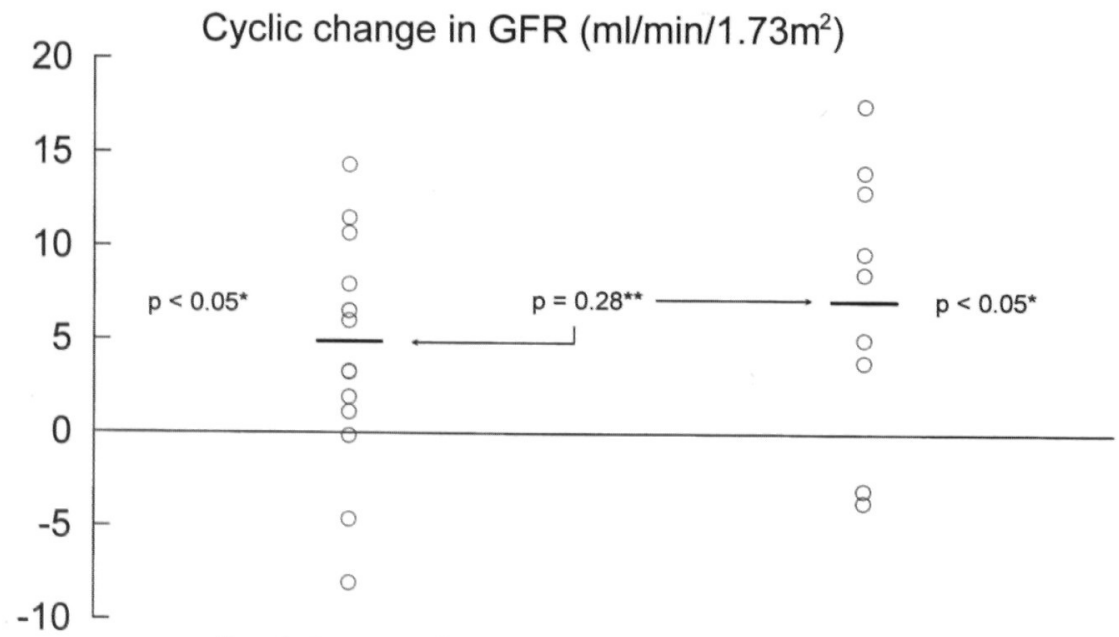

Ovulatory cycles

Anovulatory cycles

Figure 2. Changes during ovulatory and anovulatory cycles in systemic vascular resistance (dyn.s/cm $\left./ 1.73 \mathrm{~m}^{2}\right)\left(\right.$ a), glomerular fitration rate $\left(\mathrm{ml} / \mathrm{min} / 1.73 \mathrm{~m}^{2}\right)$ (b), effective renal plasma flow $\left(\mathrm{ml} / \mathrm{min} / 1.73 \mathrm{~m}^{2}\right)$ (c) and filtration fraction (d). * Wilcoxon Matched-Pairs Signed Ranks Test, ** Mann-Whitney-U Test. 
(c)

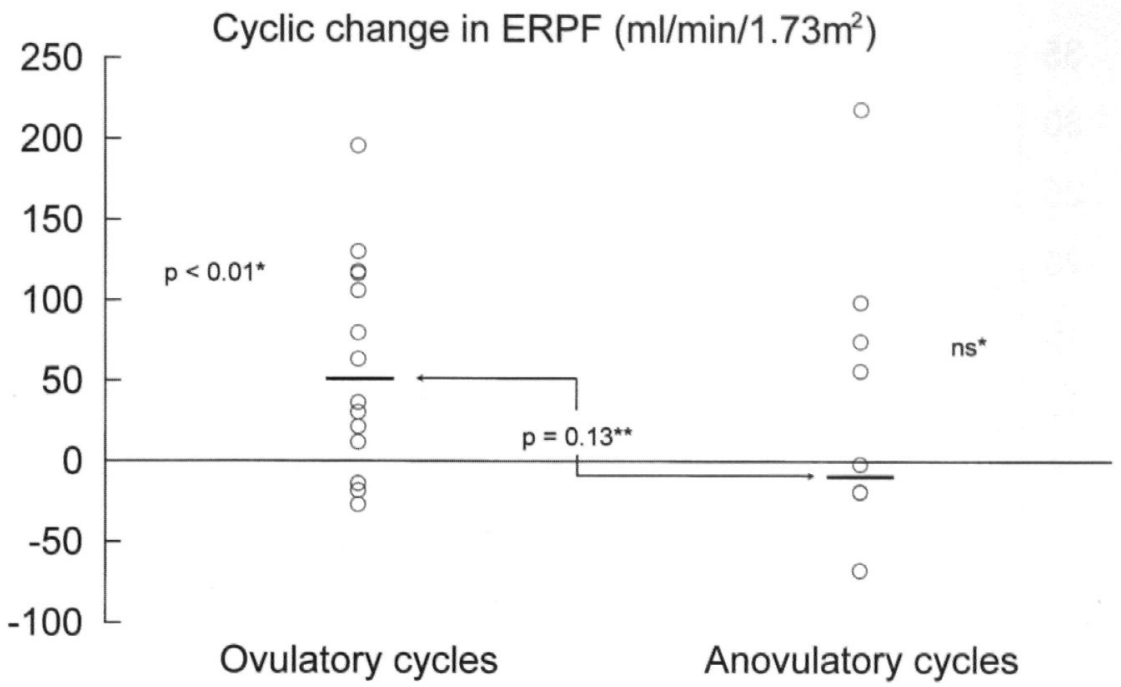

(d)

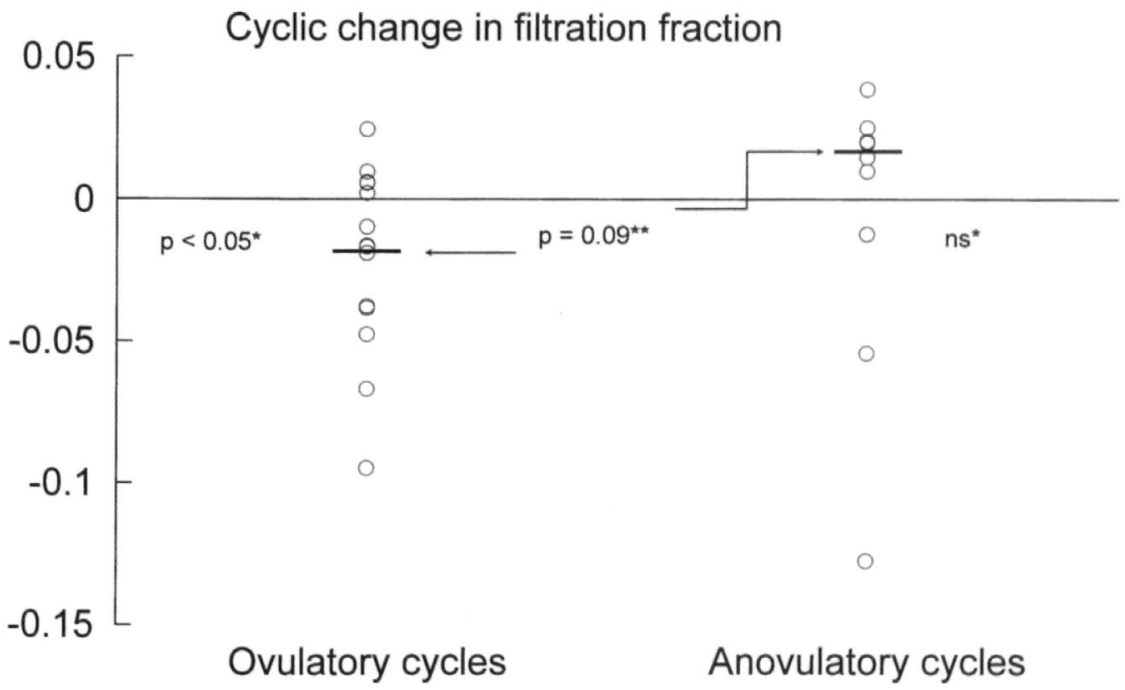

Continued figure 2. 

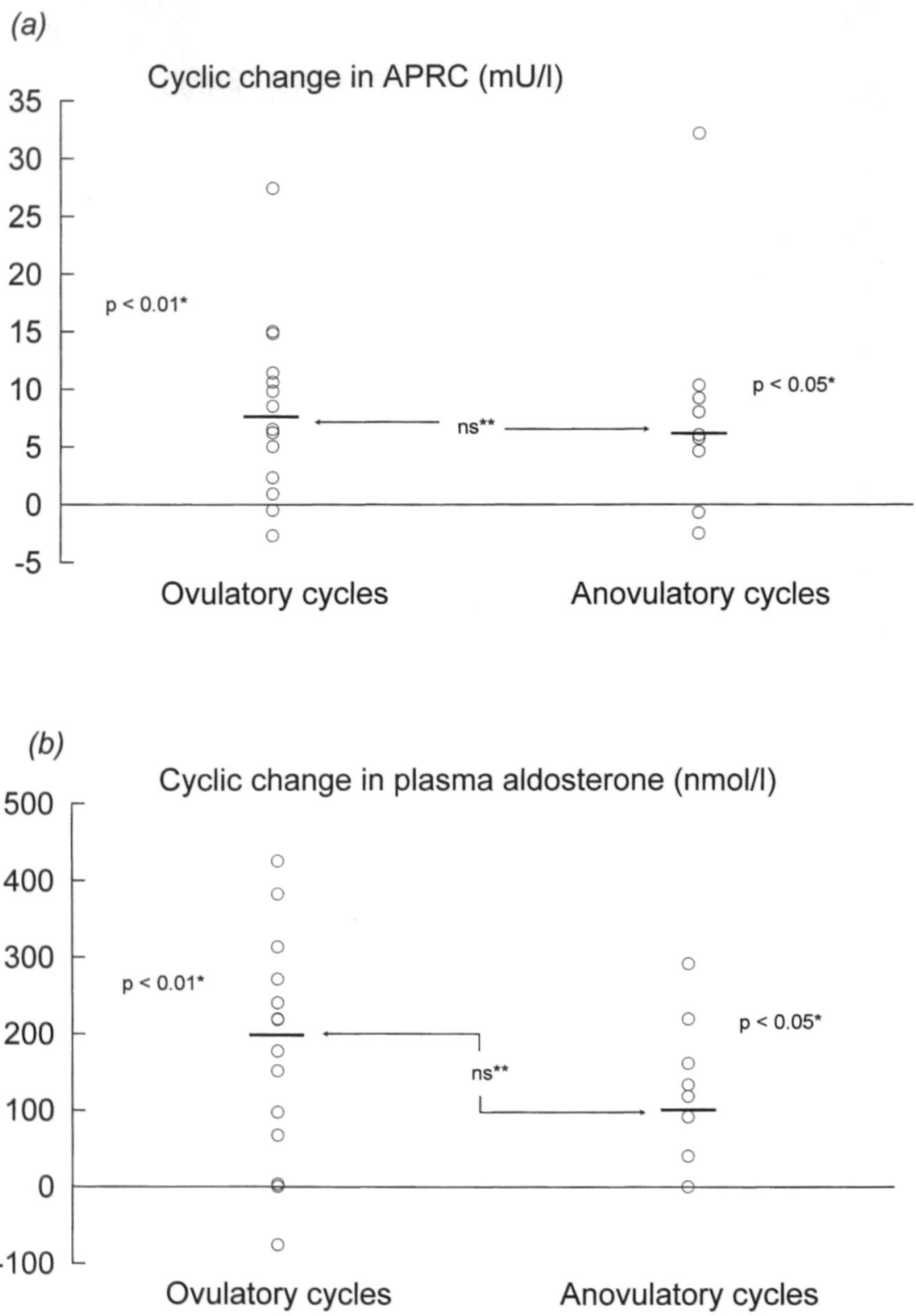

Figure 3. Changes during ovulatory and anovulatory cycles in activated plasma renin concentration $(\mathrm{mU} / \mathrm{l})(\mathrm{a})$ and plasma aldosterone concentration (nmol/l) (b). * Wilcoxon Matched-Pairs Signed Ranks Test, ** Mann-Whitney-U Test. 
Table 3. Volume homeostasis and neurohormones of subjects during ovulatory and anovulatory menstrual cycles.

\begin{tabular}{|c|c|c|c|c|c|}
\hline & \multicolumn{2}{|c|}{ Ovulatory cycle $(n=14)$} & \multicolumn{3}{|c|}{ Anovulatory cycle $(n=8)$} \\
\hline & $\mathrm{FP}^{+}$ & $L P-F P \neq$ & $\mathrm{FP}^{\dagger}$ & $L P-F P^{\ddagger}$ & $p$-value ${ }^{\S}$ \\
\hline $\begin{array}{l}\text { PV } \\
(\mathrm{m} / \mathrm{kg} \mathrm{lbm})\end{array}$ & $\begin{array}{l}54.0 \\
(48.7-57.1)\end{array}$ & $\begin{array}{l}-0.8 \\
(-3.5 \leftrightarrow+3.9)\end{array}$ & $\begin{array}{l}52.0 \\
(48.5-54.2)\end{array}$ & $\begin{array}{l}+0.9 \\
(-2.3 \leftrightarrow+4.9)\end{array}$ & $p=0.37$ \\
\hline $\mathrm{Ht}$ & $\begin{array}{l}0.35 \\
(0.33-0.37)\end{array}$ & $\begin{array}{l}+0.00 \\
(-0.02 \leftrightarrow+0.01)\end{array}$ & $\begin{array}{l}0.35 \\
(0.34-0.36)\end{array}$ & $\begin{array}{l}+0.01 \\
(-0.04 \leftrightarrow+0.03)\end{array}$ & $p=0.74$ \\
\hline $\begin{array}{l}\text { BMI } \\
\left(\mathrm{kg} / \mathrm{m}^{2}\right)\end{array}$ & $\begin{array}{l}21.3 \\
(19.8-24.4)\end{array}$ & $\begin{array}{l}-0.1 \\
(-0.4 \leftrightarrow+0.5)\end{array}$ & $\begin{array}{l}21.3 \\
(20.3-22.7)\end{array}$ & $\begin{array}{l}-0.01 \\
(-0.6 \leftrightarrow+0.7)\end{array}$ & $p=0.89$ \\
\hline $\begin{array}{l}\text { Osmolality } \\
\text { (mosm } / \mathrm{kg} \text { ) }\end{array}$ & $\begin{array}{l}282 \\
(278-284)\end{array}$ & $\begin{array}{l}-5.0 \\
(-9.0 \leftrightarrow+2.0)\end{array}$ & $\begin{array}{l}282 \\
(280-283)\end{array}$ & $\begin{array}{l}-2.0 \\
(-8.0 \leftrightarrow+4.0)\end{array}$ & $p=0.27$ \\
\hline $\begin{array}{l}\text { Serum albumin } \\
(g / 1)\end{array}$ & $\begin{array}{l}38.9 \\
(36.8-40.2)\end{array}$ & $\begin{array}{l}-1.0^{*} \\
(-2.5 \leftrightarrow-0.1)\end{array}$ & $\begin{array}{l}40.1 \\
(38.9-41.3)\end{array}$ & $\begin{array}{l}-1.2 \\
(-3.6 \leftrightarrow+5.6)\end{array}$ & $p=0.63$ \\
\hline $\begin{array}{l}\text { APRC } \\
(m \cup / l)\end{array}$ & $\begin{array}{l}18.1 \\
(10.7-24.6)\end{array}$ & $\begin{array}{l}+7.5 * * \\
(+0.9 \leftrightarrow+14.8)\end{array}$ & $\begin{array}{l}8.5 \\
(6.3-14.7)\end{array}$ & $\begin{array}{l}+5.9^{\star} \\
(+0.5 \leftrightarrow+32.1)\end{array}$ & $p=0.49$ \\
\hline $\begin{array}{l}\text { Angll } \\
\text { (pmol/l) }\end{array}$ & $\begin{array}{l}7.8 \\
(6.1-10.8)\end{array}$ & $\begin{array}{l}+1.3^{*} \\
(+0.1 \leftrightarrow+3.1)\end{array}$ & $\begin{array}{l}8.2 \\
(7.4-9.4)\end{array}$ & $\begin{array}{l}+1.1 \\
(-3.0 \leftrightarrow+4.4)\end{array}$ & $p=0.54$ \\
\hline $\begin{array}{l}\text { Aldo } \\
\qquad(\mathrm{nmol} / 1)\end{array}$ & $\begin{array}{l}97.5 \\
(10.0-193)\end{array}$ & $\begin{array}{l}+198^{\star \star} \\
(+3.0 \leftrightarrow+313)\end{array}$ & $\begin{array}{l}42.0 \\
(10.0-119)\end{array}$ & $\begin{array}{l}+105^{\star} \\
(+10.0 \leftrightarrow+291)\end{array}$ & $p=0.26$ \\
\hline $\begin{array}{l}\text { ANP } \\
\qquad(\mathrm{pg} / \mathrm{ml})\end{array}$ & $\begin{array}{l}111.5 \\
(52.8-147.0)\end{array}$ & $\begin{array}{l}-32.0 \\
(-50.0 \leftrightarrow+12.0)\end{array}$ & $\begin{array}{l}98.5 \\
(88.0-120.7)\end{array}$ & $\begin{array}{l}-9.5 \\
(-73.3 \leftrightarrow+73.0)\end{array}$ & $p=0.78$ \\
\hline
\end{tabular}

Medians and interquartile ranges of the $\mathrm{FP}^{\dagger}$, and median differences (with $95 \%$ confidence interval) between LP and FP ${ }^{\ddagger}$ are listed. Changes in variables during the menstrual cycle were evaluated using the Wilcoxon matched-pairs signed ranks test $\left({ }^{*} p<0.05,{ }^{* *} p<0.01\right.$ ). Differences in cyclic changes between ovulatory and anovulatory cycles were analyzed by the Mann-Whitney-U test ${ }^{\S}$. FP, follicular phase; LP, luteal phase; PV, plasma volume; Ibm, lean body mass; Ht, hematocrit; BMI, body mass index; APRC, active plasma renin concentration; Angll, plasma angiotensin II; Aldo, plasma aldosterone; ANP, atrial natriuretic peptide.

\section{DISCUSSION}

The objective of the present study was to determine the individual roles of progesterone and $17 \beta$-estradiol in the hemodynamic changes observed during the menstrual cycle. Cyclic central and peripheral hemodynamic changes in ovulatory women were compared with those in anovulatory women. Because in our previous reports 125,127 , we only observed cyclic hemodynamic changes in women on a high-salt diet, all subjects were put on a diet containing $220 \mathrm{mmol}$ of sodium per day. Our data show that, except for a higher serum $17 \beta$-estradiol in the ovulatory group, follicular values of all variables in both groups were comparable. Of all the 
measured variables, only the cyclic changes in serum progesterone, ERPF, FF and RF differed between the two groups. Although these differences between both groups were small, their combination brings up for some interesting inferences. In both groups the GFR was higher in the "LP" of the menstrual cycle, but only in the ovulatory group this increase was paralleled by a rise in ERPF and RF, and a fall in FF and RVR (Table 2 and Fig.2). Although, the behaviour of the reninangiotensin-aldosterone (RAA) system was comparable in both groups, the magnitude of the cyclic changes was somewhat greater during ovulatory cycles than during anovulatory cycles (Table 3 and Fig.3).

A tendency of the RAA-system to become more activated during ovulatory cycles than during anovulatory cycles may be seen as a compensation for the natriuretic effect of progesterone ${ }^{13-15}$, resulting in no detectable changes in PV and plasma ANP levels in both types of cycles. Oparil et al. ${ }^{122}$ described that, next to competitive inhibition of aldosterone at the distal tubulus, the diuretic effect of progesterone may be related to changes in renal hemodynamics. After intramuscular administration of progesterone in male subjects they observed an increase in ERPF, a stable GFR and a decrease in FF. Theoretically, a lower FF will lead to a lower peritubular oncotic pressure and, hence, increased reabsorption of sodium in the proximal tubule. At any rate, our observations on cyclic changes in renovascular dynamics in ovulatory cycles compared to anovulatory cycles, resemble those reported by Oparil et al. ${ }^{122}$ with respect to the renal effects of progesterone. It is conceivable, that analogous to the situation in pregnancy, progesterone decreases the sensitivity for AngII, a potent vasoconstrictor of the efferent arteriole, thereby causing the above mentioned renal hemodynamic effects. Although this could explain the decrease in RVR, it does not sufficiently account for the rise in GFR. However, it is not known to what extent the interplay between progesterone, $17 \beta$-estradiol and AngII modulates the ratio between vascular resistances of the afferent and efferent arterioles. Interestingly, Kalenga and co-workers reported down-regulation of the angiotensin AT1 receptor in human trophoblast cells by progesterone but not by $17 \beta$-estradiol ${ }^{129}$. It is tempting to speculate that progesterone and not $17 \beta$-estradiol also triggers down-regulation of the angiotensin AT1 receptor in the efferent arteriole of the glomerulus.

The lack of an increase in GFR in response to progesterone ${ }^{122}$ together with our observation of an increase in GFR also in anovulatory cycles suggests that the rise in GFR is primarily estrogen-mediated. The fact that no correlation was found between changes in GFR and changes in serum $17 \beta$-estradiol indicate that the relationship between these two variables is probably complex.

In-vitro studies using human and animal arteries showed that both progesterone alone and $17 \beta$-estradiol alone can cause vascular relaxation ${ }^{78-80}$. We observed that the luteal decline in TPR in the ovulatory group and anovulatory group is comparable. To our knowledge TPR has never been studied in vivo in human 
anovulatory menstrual cycles. It seems that in vivo, $17 \beta$-estradiol, and not progesterone, is the major mediator in causing menstrual cycle related vascular relaxation. The observed "luteal" increase in FBF during anovulatory cycles only, is compatible with this concept.

In conclusion, except for a difference in cyclic change in serum progesterone, ERPF, FF and RF, we did not find statistical significant differences in cyclic hemodynamic changes between ovulatory and anovulatory menstrual cycles. Although in many instances both steroids may act in concert in inducing the hemodynamic changes with the menstrual cycle, the results of this study suggest that the luteal fall in systemic vascular resistance and the rise in GFR are primarily a $17 \beta$-estradiol effect, whereas, the luteal rise in ERPF is a selective progesterone effect. Whether the latter is a consequence of progesterone induced downregulation of the angiotensin AT1 receptor in the efferent arteriole of the glomerulus, remains to be elucidated. 

7 CHAPTER

Persistent abnormalities in plasma volume and renal hemodynamics in patients with a history of preeclampsia

Erik van Beek*, M.D.,Timo H.A. Ekhart*, Paul M.H. Schiffers**, Ph.D., Jim van Eyck***, M.D., Ph.D., Louis L.H. Peeters*, M.D., Ph.D, Peter W. de Leeuw****, M.D., Ph.D.

Departments of Obstetrics and Gynecology*, and Internal Medicine****, University Hospital Maastricht, and department of Pharmacology**, University of Maastricht, Maastricht, and Departments of Obstetrics and Gynecology***, Sophia Hospital, Zwolle, The Netherlands.

Presented in part at the seventeenth Annual Meeting of the Society of Perinatal Obstetricians, Anaheim, California, January 20-25, 1997

Am J Obstet Gynecol 1998; 179: 690-6 
Objective: The objective was to test the hypothesis that women with a recent history of preeclampsia have abnormalities in renal hemodynamics and volume status.

Study Design: We studied a group of 26 primiparous ex-preeclamptics (ex-PE group) and a group of 12 parous women with a history of uneventful pregnancies (controls). At least four months post-partum we compared the following variables between these groups: effective renal plasma flow (ERPF), glomerular filtration rate (GFR), plasma volume (PV) and plasma concentrations of active renin (APRC), angiotensin II (AngII), aldosterone (Aldo) and atrial natriuretic peptide (ANP).

Results: Both PV and ANP were lower in the ex-PE group. As compared with the controls, the ex-PE group also had a lower ERPF, a higher filtration fraction (FF) and a higher renal vascular resistance (RVR). Intergroup differences in APRC, AngII and Aldo were small and inconsistent.

Conclusions: Women with a history of preeclampsia are relatively hypovolemic and tend to have lower effective renal plasma flow and higher renal vascular resistance and filtration fraction than do controls. These findings support the hypothesis that otherwise healthy women with a history of preeclampsia show abnormalities in their volume status and renal hemodynamics, irrespective of their blood pressure.

\section{INTRODUCTION}

Recent evidence suggests that endothelial cell dysfunction plays a key role in the pathogenesis of preeclampsia, a clinical syndrome during the second half of pregnancy characterized by increased capillary permeability (edema, proteinuria), vasoconstriction (hypertension, renal and liver dysfunction) and activation of the clotting cascade (thrombocytopenia) ${ }^{33,130}$. The events during the first half of pregnancy that lead to both functional and morphologic abnormalities of the endothelium, although still incompletely understood, are considered to be secondary to placental ischemia, which in turn may be the consequence of poor placentation 30,32 .

Data from epidemiologic studies suggest that the cause of defective placentation is multifactorial. Next to genetic 57,60 and immunologic 42,43 predispositions, there seems to be an important role for vascular factors ${ }^{63}$. Support for vascular factors as potential triggers for preeclampsia comes from the observation that the incidence of preeclampsia is increased among women with chronic hypertension ${ }^{131}$ or diabetes mellitus ${ }^{132}$. The relative importance of each of these potential causes of 
preeclampsia and their mutual relationship can only be determined by in-depth exploration, either in the non-pregnant state or during the latent phase that precedes manifest disease. In this respect it was an important finding that among subjects with a history of severe early-onset preeclampsia, in addition to chronic hypertension, the prevalence of subclinical abnormalities in the clotting system leading to thrombophilia was found to be substantially increased ${ }^{64}$.

Whether there is also a subgroup of women with latent cardiovascular dysfunction who may be at risk for preeclampsia is unknown. Several years ago it was demonstrated that healthy, normotensive subjects with an increased risk for development of hypertension display abnormalities in their renal hemodynamics and reninangiotensin-aldosterone system ${ }^{133}$.

This study was therefore designed to test the hypothesis that symptom-free, apparently healthy women with a history of preeclampsia have abnormalities in their renal hemodynamics and volume status. To this end we compared the effective renal plasma flow (ERPF), glomerular filtration rate (GFR), plasma volume (PV) and the plasma concentrations of active renin (APRC), angiotensin II (AngII), aldosterone (Aldo) and atrial natriuretic peptide (ANP) between two groups of parous women with or without previous preeclampsia.

\section{MATERIAL AND METHODS}

\section{Subjects}

Experiments were performed in 26 primiparous women with a history of preeclampsia ${ }^{1}$ (ex-PE group) and 12 subjects with a history of uneventful pregnancies (control group). Women with a history of preeelampsia were selected from the outpatient departments of two tertiary referral centers (University Hospital Maastricht and Sophia Hospital in Zwolle), whereas subjects from the control group were recruited by an advertisement in a local newspaper. Contraindications for recruitment were lactation and the use of any medication. Subjects' characteristics are listed in Table 1. All participants gave written informed consent, and the study was approved by the hospital's medical ethics committee. Measurements were performed at least four months post partum (mean \pm $\mathrm{SD}$ of ex-PE - and control group were $7 \pm 2$ and $41 \pm 35$ months, respectively) and in the midluteal phase of the menstrual cycle.

\section{Protocol}

All subjects were asked to adhere to their usual dietary regimen in the days before the study. They collected a 24-hour urine sample for determination of sodium excretion on the day before the study. All measurements were performed in the morning on two consecutive days. Data acquisition was started after an acclimati- 
zation period of at least 30 minutes with the subject in sitting (first day) or supine (second day) position. The participants were asked not to eat, smoke, or drink, except for water, during a period of ten hours before and during the measurements. PV was measured on the first day of the study, and ERPF, GFR, plasma concentrations of ANP, APRC, AngII and Aldo were determined on the second day.

\section{Measurements}

PV ( $\mathrm{ml} / \mathrm{kg}$ body mass) was assessed with the iodine 125-labeled albumin technique with a coefficient of variation (CV) of $4.2 \%$. A five $\mu \mathrm{Ci}$ dose of human albumin labeled with iodine 125 , dissolved in $1 \mathrm{ml}$ saline solution, was given intravenously (total body dose of irradiation $0.04 \mathrm{mSv}$ ). After 10, 20 and $30 \mathrm{~min}$, blood samples $(5 \mathrm{ml})$ were withdrawn to determine the volume-specific plasma radioactivity $(\mathrm{mCi} / \mathrm{ml})$. The virtual volume-specific plasma radioactivity at the time of bolus injection was calculated by extrapolation from the sequentially obtained volume-specific plasma radioactivity data determined in the three consecutive plasma samples. PV was obtained by dividing the total injected radioactivity by the virtual volume-specific plasma radioactivity at the time of bolus injection.To eliminate the impact of body fat on PV per kg body weight we calculated the body fat fraction ${ }^{128}$ to express the $\mathrm{PV}$ in $\mathrm{ml} / \mathrm{kg}$ of lean body mass as well.

Renal hemodynamic values (ERPF and GFR) were derived from the plasma clearances (continuous infusion method) of $\mathrm{PAH}(\mathrm{CV} \mathrm{8 \% )}$ ) and inulin (CV 6\%), respectively ${ }^{12}$. During the infusion of $\mathrm{PAH}$ and inulin, mean arterial pressure (MAP), systolic blood pressure (SBP), diastolic blood pressure (DBP) and heart rate (HR) were measured in the opposite arm with a semi-automatic oscillometric device (Dinamap Vital Signs Monitor 1846, Critikon, Tampa, FL, USA). The measurements during the last 30 minutes of the two-hour equilibration period were taken to calculate the average MAP, SBP, DBP and HR. Renal blood flow (RBF in $\mathrm{ml} / \mathrm{min}$ ) and renal vascular resistance (RVR in dyn.s/cm ${ }^{5}$ ) were calculated by the formulas ERPF/(1-hematocrit) and (MAP/RBF) x 80,000, respectively. Filtration fraction (FF) was obtained by taking the ratio of GFR and ERPF. All measurements were standardized for body-surface area.

APRC was assessed by the IRMA-method (Nichols Institute Diagnostics, Wychen, The Netherlands), with an intra-assay CV of $2.9 \%$ and an interassay CV of $7.6 \%{ }^{113}$. AngII was determined by radio-immunoassay (RIA) after ethanol extraction (Nichols Institute Diagnostics; intra-assay CV 4.3\% and interassay CV $7.5 \%)^{114}$. Aldo was assayed by means of a solid-phase protein binding RIA (Diagnostic Products Corporation, La, USA; intra- and interassay CV $4.3 \%$ and $6.7 \%$, resp.) ${ }^{115}$. ANP was measured by a competitive protein-binding RIA (Nichols Institute Diagnostics; intra-assay and interassay CV $7.0 \%$ and $12.9 \%$, resp.) after extraction of plasma over Sep-Pak C18 columns ${ }^{116}$. 
Statistical analysis

Before analysis the distribution of all variables was tested for normality by means of the Kolmogorov-Smirnov test. Because all variables were normally distributed, we chose to analyze differences between groups by the Student-t test. An exception was made for APRC, AngII and Aldo. Because we found in some cases levels that were below the detection limit for these variables, the Mann-Whitney-U test was used to analyze hormonal differences between groups. Differences in frequencies between groups were evaluated by the Fisher exact test. Correlations between potentially related variables were tested by Spearman's rank correlation analysis. In the comparisons a p-value of less than $5 \%$ was considered statistically significant.

\section{RESULTS}

On average, MAP was higher in the ex-PE group than in the controls $(\mathrm{p}<0.01$; Table 1). Data for renal hemodynamics, PV and plasma concentrations of ANP are listed in Table 2; plasma concentrations of APRC, AngII and Aldo are depicted in Table 3. Compared with the control group, ERPF was significantly lower, and FF and RVR were significantly higher in the ex-PE group. GFR did not differ significantly between the two groups. Both PV and plasma concentrations of ANP were significantly lower in the ex-PE group than in the control group. Because no difference in hematocrit between the groups was found, blood volume was also lower in the ex-PE group. There were no significant differences between the ex-PE group and the control group with respect to the renin-angiotensinaldosterone system.

Because differences in variables between groups could be related to differences in duration of the postpartum period, age and MAP, we also calculated possible correlations. Within the ex-PE group no correlation was found between PV (Fig 1), ANP, ERPF or APRC and the duration of the postpartum period. ERPF was inversely related to age in the ex-PE patients $(r=-0.41, \mathrm{p}=0.04)$ but not in the controls. PV, ANP and APRC were not related to age in either group. APRC was inversely correlated with MAP $(r=-0.41, \mathrm{p}=0.04)$ and with $\mathrm{PV}(\mathrm{ml} / \mathrm{kg}$ lean body mass; $r=-0.52, p=0.01$ ) in ex-PE patients only. When tested in a stepwise multiple regression model, only PV remained as a significant determinant of APRC. There were no statistically significant correlations between PV, ANP or ERPF and MAP in either group, nor were there significant correlates between PV, ANP or ERPF and gestational age at delivery in the ex-PE group.

To be able to perform a post-hoc comparison between a normotensive, ex-PE subgroup (ex-PE, normotensive) and the controls, we selected those patients $(n=16)$ who had a MAP below the mean +2 SD of that of the control group ( $<96 \mathrm{mmHg}$ ). 
Table 1. Characteristics of both groups of subjects. *

$\begin{array}{lll}\text { characteristic } & \begin{array}{l}\text { Controls } \\ (n=12)\end{array} & \begin{array}{l}\text { Ex-preeclamptics } \\ (n=26)\end{array} \\ \text { Age }(\mathrm{yr}) & 33 \pm 2 & 29 \pm 4^{\ddagger} \\ \text { Primiparity }(\mathrm{n}) & 3(25) & 26(100)^{5} \\ \text { Body mass index }\left(\mathrm{kg} / \mathrm{m}^{2}\right) & 22 \pm 4 & 24 \pm 3 \\ \text { BMI }>26 \mathrm{~kg} / \mathrm{m}^{2}(\mathrm{n}) & 1(8) & 7(27) \\ \text { GA at delivery }(\mathrm{n}<32 \mathrm{wk}) & 0 & 13(50)^{\S} \\ \text { HELLP }(\mathrm{n}) & 0 & 20(77)^{\S} \\ \text { IUGR }\left(\mathrm{n}<\mathrm{P}_{10}\right) & 0 & 12(46)^{\S} \\ \text { SBP }(\mathrm{mmHg}) & 112 \pm 8 & 124 \pm 18^{\dagger} \\ \text { DBP }(\mathrm{mmHg}) & 66 \pm 9 & 79 \pm 14^{\ddagger} \\ \text { DBP }>90 \mathrm{mmHg}(\mathrm{n}) & 0 & 3(12) \\ \text { MAP }(\mathrm{mmHg}) & 82 \pm 7 & 94 \pm 13^{\ddagger} \\ \text { Heart rate }(\text { beats } / \mathrm{min}) & 67 \pm 11 & 64 \pm 8 \\ \text { Urinary sodium }(\mathrm{mmol} / 24 \mathrm{hr}) & 139 \pm 40 & 138 \pm 42\end{array}$

* Data are either listed as means \pm SD or as absolute numbers with percentage between parenthesis. ${ }^{\dagger} p<0.05,{ }^{\ddagger} p<0.01$, for the comparisons with controls (Student-t test). ${ }^{\S} p<0.05$ for the comparisons with controls (Fisher exact probability test).

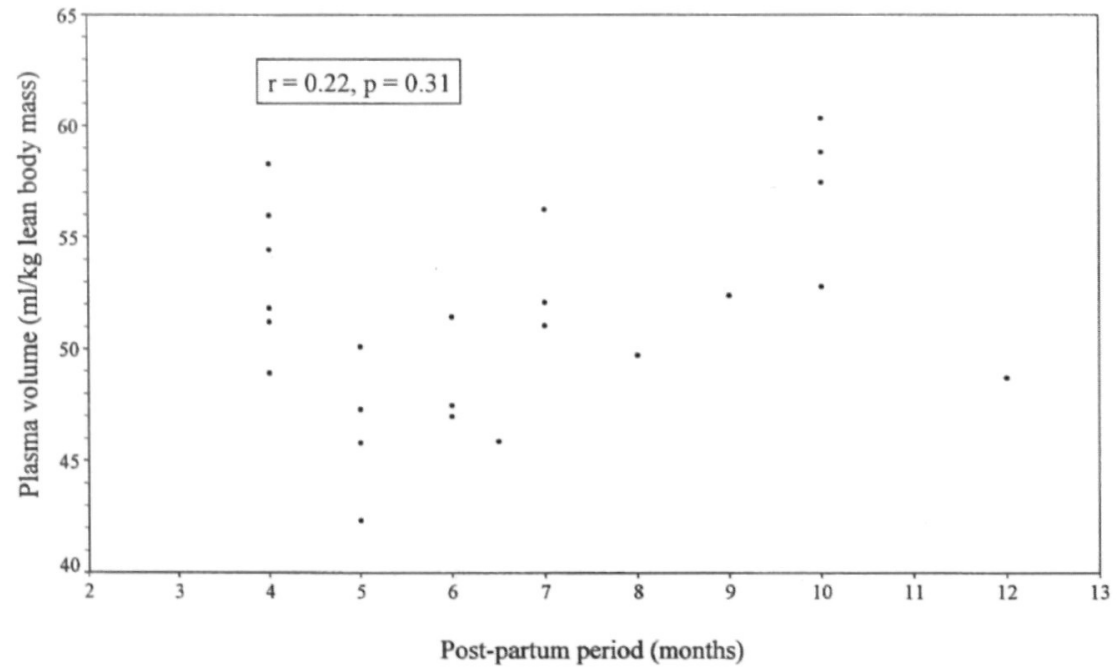

Figure 1. Scatterplot illustrating the lack of correlation between plasma volume $(\mathrm{ml} / \mathrm{kg}$ of lean body mass) and duration of postpartum period (months) in 24 ex-PE subjects. In two of the 26 exPE subjects enrolled we could not measure plasma volume for logistic reasons. 
Table 2. Characteristics for renal function and volume status in both groups of subjects (means $\pm \mathrm{SD}$ ).

\begin{tabular}{|c|c|c|}
\hline Characteristic & $\begin{array}{l}\text { Controls } \\
(n=12)\end{array}$ & $\begin{array}{l}\text { Ex-preeclamptics } \\
(n=26)\end{array}$ \\
\hline $\begin{array}{l}\text { Effective renal plasma flow } \\
\left(\mathrm{ml} / \mathrm{min} / 1.73 \mathrm{~m}^{2}\right)\end{array}$ & $553 \pm 67$ & $482 \pm 88^{\dagger}$ \\
\hline $\begin{array}{l}\text { Glomerular filtration rate } \\
\left(\mathrm{ml} / \mathrm{min} / 1.73 \mathrm{~m}^{2}\right)\end{array}$ & $121 \pm 21$ & $134 \pm 19$ \\
\hline $\begin{array}{l}\text { Filtration fraction } \\
\text { (GFR/ERPF) }\end{array}$ & $0.22 \pm 0.03$ & $0.28 \pm 0.04^{\S}$ \\
\hline $\begin{array}{l}\text { Renal vascular resistance } \\
\left(\text { dyn. } 5 / \mathrm{cm}^{5}\right)\end{array}$ & $7490 \pm 868$ & $9981 \pm 2468^{\S}$ \\
\hline $\begin{array}{l}\text { Plasma volume } \\
(\mathrm{ml} / \mathrm{kg})\end{array}$ & $40 \pm 3$ & $36 \pm 4^{\ddagger}$ \\
\hline $\begin{array}{l}\text { Plasma volume } \\
\text { ( } \mathrm{ml} / \mathrm{kg} \text { lean body mass) }\end{array}$ & $55 \pm 3$ & $52 \pm 5^{\dagger}$ \\
\hline $\begin{array}{l}\text { Atrial natriuretic peptide } \\
(\mathrm{pg} / \mathrm{ml})\end{array}$ & $68 \pm 26$ & $38 \pm 19^{\ddagger}$ \\
\hline Hematocrit & $0.38 \pm 0.02$ & $0.38 \pm 0.02$ \\
\hline
\end{tabular}

Table 3. Characteristics of the renin-angiotensin-aldosterone system in both groups of subjects (medians and interquartile ranges).

\begin{tabular}{lll} 
Characteristic & $\begin{array}{l}\text { Controls } \\
(n=12)\end{array}$ & $\begin{array}{l}\text { Ex-preeclamptics } \\
(\mathrm{n}=26)\end{array}$ \\
\hline $\begin{array}{l}\text { Active plasma renin concentration (mU/l) } \\
\text { Plasma angiotensin II (pmol//) }\end{array}$ & $16.2(9.7-19.7)$ & $16.5(10.8-22.2)$ \\
Plasma aldosterone (pmol/l) & $10.9(10.5-14.0)$ & $11.3(6.7-16.8)$ \\
\hline
\end{tabular}

${ }^{\dagger} p<0.05$ for the comparisons with controls (Mann-Whitney $U$ test). 


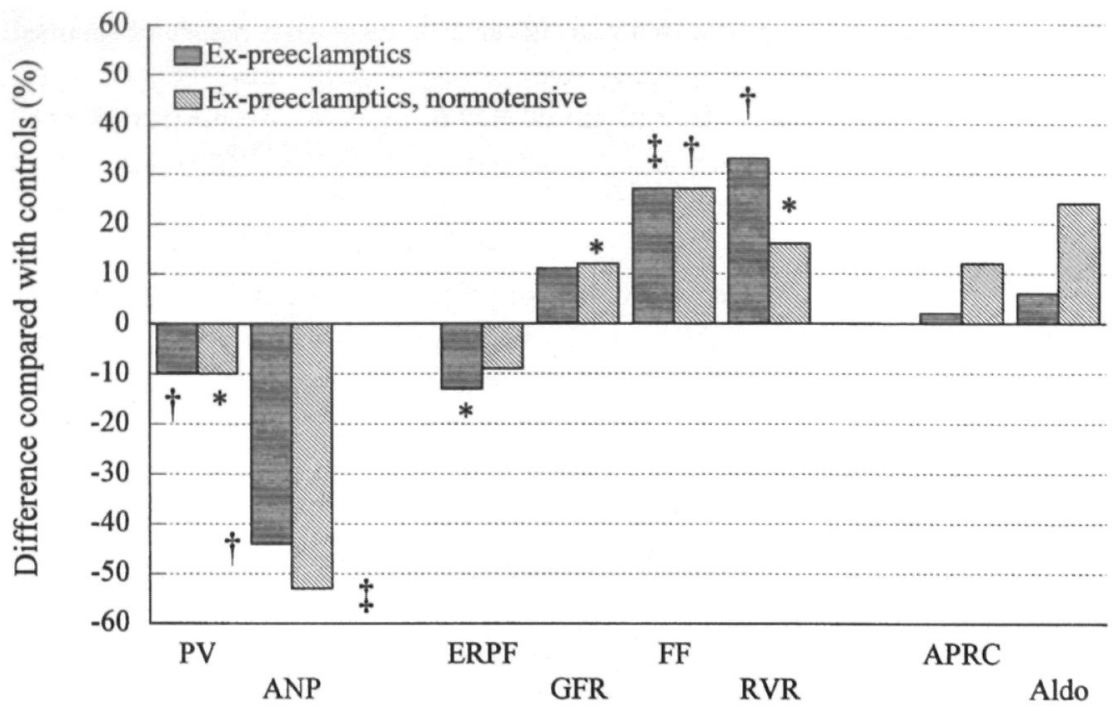

Figure 2. Differences (\%) from controls in plasma volume (PV), atrial natriuretic peptide (ANP), effective renal plasma flow (ERPF), glomerular filtration rate (GFR), filtration fraction (FF), renal vascular resistance (RVR), active plasma renin concentration (APRC) and plasma aldosterone (Aldo).

${ }^{*} P<0.05,+P<0.01, \neq P<0.001$ for the comparisons with controls (Student-t test).

The MAP of this normotensive ex-PE group $(86 \mathrm{mmHg}$ ) was comparable with the MAP of the controls $(82 \mathrm{mmHg})$. The relevant variables are shown in Fig 2. The lower ERPF in the normotensive ex-PE group just failed to reach statistical significance when compared with controls $\left(504\right.$ vs. $553 \mathrm{ml} / \mathrm{min} / 1.73 \mathrm{~m}^{2}, \mathrm{p}=$ $0.07)$. GFR, FF and RVR, on the other hand, were significantly higher in the normotensive ex-PE group than in the control group. Both PV and plasma ANP concentration were significantly lower in the normotensive ex-PE group than in the control group. Plasma concentrations of APRC, AngII and Aldo did not differ between the normotensive ex-PE group and the control group.

\section{COMMENT}

It is still common practice to subdivide preeclampsia into a "superimposed" and a "pure" phenotype, depending on whether the patient with preeclampia had preexisting hypertension ${ }^{1}$. However, there is increasing evidence to support the view that the concept of "pure" preeclampsia must be abandoned. For instance the recent discovery of thrombophilia, associated with latent abnormalities in hemosta- 
sis, in more than half of the women with a history of severe early-onset preeclampsia indicates that many such patients with allegedly "pure" preeclampsia in fact have other (subclinical) abnormalities ${ }^{64}$. The objective of this study was to examine ex-preeclamptic women for latent abnormalities in volume status and renal hemodynamics.

Renal biopsy studies in patients with preeclampsia have shown that merely $24 \%$ of multiparous women, as opposed to $76 \%$ of primiparous women, have glomerular endotheliosis, the histological hallmark of preeclampsia, as the only lesion ${ }^{134}$. We therefore included in our study only primiparous women with a history of preeclampsia, as defined by the US National Institutes of Health Working Group on Hypertension in Pregnancy ${ }^{1}$.

Our data show that even several months after delivery the PV of the ex-PE group was $10 \%$ lower than that of a control group. Moreover, compared with the control group, ERPF was lower and FF and RVR were higher in the ex-PE group.

In general, it is advisable to standardize dietary sodium intake in studies on renal hemodynamics or the renin-angiotensin-aldosterone system. For practical reasons food intake of the subjects in this study was not standardized, but all participants were asked to refrain from excessive salt intake and to adhere to their usual dietary regimen on the days before the study. Although not ideal, this practice resulted in comparable 24-hour urinary sodium excretions in the two groups. We are therefore quite confident that the observed differences in renal hemodynamics between the two groups are genuine.

Because we found an inverse correlation $(r=-0.41)$ between ERPF and age in the ex-PE group, a possible bias in the comparisons could be different mean ages of the subjects in the groups under investigation. However, the potential effect of the four years' difference in mean age between the subjects of the ex-PE group and the control group is likely to be small. In addition, had the ex-PE group been four years older, the difference in ERPF would have been even greater. It is therefore most likely that ERPF is indeed lower in patients with a history of preeclampsia. Likewise, FF and RVR are higher in this group of patients. Although this observational study was not designed to explore the underlying mechanism of these changes, one could speculate that a decreased circulatory volume in expreeclamptics might have caused the lower renal blood flow.

In patients with essential hypertension or in people at risk for the development of hypertension, abnormal renal hemodynamics such as those found in this study are usually associated with a suppressed renin-angiotensin-aldosterone system ${ }^{133}$. In our study, however, we could not find significant differences in APRC, AngII or Aldo between the ex-PE group and the control group. Yet APRC was inversely related to PV in ex-PE patients, indicating that in this group some stimulation of renin occurred as a result of hypovolemia. However, any stimulating effect on the 
renin-angiotensin-aldosterone system was probably counterbalanced by concurrent suppression of renin as a result of higher blood pressure.

The International Committee for Standardization in Haematology (ICSH) stated, in its report about recommended methods for measurements of red blood cell volume and PV, that PV in healthy subjects measured by the radioiodinelabeled albumin dilution technique amounts approximately $40 \mathrm{ml} / \mathrm{kg}$ in both sexes ${ }^{135}$. In fact, we found exactly the same value in our control population. Nevertheless, because the correlation of PV is closer with lean body mass than with body weight, we expressed our PV data not only as $\mathrm{ml} / \mathrm{kg}$ of body weight but also as $\mathrm{ml} / \mathrm{kg}$ of lean body mass ${ }^{135}$. With both methods we obtained similar results, a significantly lower PV in the ex-PE group. The data therefore strongly suggest that women with a history of preeclampsia are unable to maintain a normal PV, at least during the first year after delivery.

Although it is widely acknowledged that preeclampsia resolves soon after delivery, this study does not rule out the possibility that the observed changes in renal hemodynamics and volume status are a result of preeclampsia. In other words, the syndrome itself may cause permanent structural damage to the vasculature, including that of the kidney ${ }^{136}$. It is also possible that recovery takes more than four months. However, the lack of a relationship between the length of the recovery time post partum and the severity of the observed abnormalities makes the latter explanation less likely. Although ideally one would perform a prospective study to rule out these theoretic possibilities, the low incidence of preeclampsia in nulliparous makes such a study practically difficult to execute.

In our opinion the so-called "pure" phenotype of preeclampsia is best evaluated in a direct comparison with a demographically similar control group matched for blood pressure. Because this could only be done in a prospective study design, which for obvious reasons is not feasible, we chose to analyze our data by defining a "pure" preeclampsia group (normotensive ex-PE group) with a MAP that fell within the mean $\pm 2 \mathrm{SD}$ of that of the control group. We decided not to split up the ex-PE group into subgroups on the basis of the severity of the syndrome because there was no correlation between gestational age at delivery (which is generally considered to reflect the severity of the syndrome) and any of the measured variables. In the normotensive ex-PE group PV was again 10\% lower than in controls, a statistically significant difference. The data on renal hemodynamics took an intermediate position between the control group and the ex-PE group with higher pressures. Still, even in the normotensive ex-PE group, GFR, FF and RVR were significantly increased with respect to these variables in the control group (Fig 2). Likewise, ANP was significantly reduced in the normotensive ex-PE group; again there were no differences with respect to APRC, AngII and Aldo. If we assume that the observed changes in volume status and renal hemodynamics precede the development of preeclampsia, it is tempting to speculate about the ex- 
istence of a group of patients with subclinical vascular diseases that have relative hypovolemia in common. Because of their relatively low blood volume or latent vascular abnormalities, the adaptive response of this group to pregnancy may differ from that seen in healthy women. The physiologic drop in vascular tone during the first trimester ${ }^{137}$ could be a stressful event for these subjects because the compensatory mobilization of blood from the venous compartment to meet the higher demands for venous return may be inadequate. This in turn may trigger a redistribution of cardiac output at the expense of the implantation site. In this respect the recently observed state of sympathetic overactivity in preeclamptics seems to be consistent with this thought ${ }^{138}$. Instead of displaying vascular relaxation in combination with a compensatory rise in circulatory volume during the first trimester, these subjects could respond to the early pregnancy-related vasorelaxing stimulus either by a redistribution of the cardiac output away from the uterus or by an impaired vascular relaxation. Both phenomena would lead to a relatively hypoperfused implantation site, causing defective trophoblast development and thus initiating the chain of events eventually culminating into placental ischemia. Placental ischemia indeed does play a key role in the pathogenesis of preeclampsia. On the basis of our findings of a reduced PV and suppressed levels of ANP, we determined that women with a history of preeclampsia are relatively hypovolemic, even after delivery. Compared with values from women with a history of uncomplicated pregnancies, their renal blood flow is lower and their filtration fraction and renal vascular resistance are higher. These findings support the notion that women with a history of preeclampsia have subclinical alterations in volume status and renal hemodynamics, even when they are normotensive. Whether these changes play an important role in the pathogenesis of preeclampsia remains to be established. 

8 CHAPTER

Changes in hemodynamics and volume homeostasis with the menstrual cycle in women with a history of preeclampsia

M.E.A. Spaanderman*, E. van Beek*, T.H.A. Ekhart*, J. van Eyck**, E.C. Cheriex***, P.W. de Leeuw****, L.L.H. Peeters* $^{* *}$

Departments of Obstetrics and Gynecology*, Cardiology ${ }^{* * *}$ and Internal Medicine****, University Hospital Maastricht, Maastricht, and department of Obstetrics and Gynecology**, Sophia Hospital, Zwolle, The Netherlands.

Submitted 


\section{ABSTRACT}

Among ex-preeclamptic (ex-PE) women the prevalence of hemodynamic and clotting disorders is raised. In this study we tested the hypothesis that the normal cyclicity in hemodynamics and renal function with the menstrual cycle, as seen in healthy women, is preserved in ex-PE subjects irrespective of whether or not they had an underlying hemodynamic or clotting disorder.

We compared the hemodynamic and volume cyclicity during the menstrual cycle in ex-PE women $(n=39)$ with that in normal parous controls $(n=10)$. The exPE's were subdivided into women with hypertension ( $\mathrm{HT}, \mathrm{n}=10)$, women with a thrombophilic disorder (TP, $\mathrm{n}=17)$ and women without these abnormalities (REST, $\mathrm{n}=12$ ). We measured at least 5 months postpartum, once during the follicular phase (FP, day $5 \pm 2$ ) and once during the luteal phase (LP, day $22 \pm 2$ ) of the menstrual cycle, the following variables: body weight and length, mean arterial pressure, heart rate (HR), cardiac output (CO), plasma volume (PV), glomerular filtration rate (GFR), effective renal plasma flow (ERPF), renal volume homeostatic hormones, reproductive hormones and catecholamines. From the measured data, we calculated body mass index (BMI), cardiac index (CI), left ventricular work, total peripheral (TPR) and renal vascular resistance (RVR), effective renal blood flow (ERBF) and renal filtration fraction (FF).

HT differed from controls by a higher baseline (FP) value for CO, CI, LVW, RVR, ANP and norepinephrine. REST differed from controls by a lower baseline $\mathrm{PV}$ and a higher baseline CO and LVW. Women in the TP group were comparable with respect to baseline hemodynamics and renal function, except for a higher RVR. In controls, HR, PV, ERPF, ERBF, volume regulatory hormones and norepinephrine in the FP had increased in the LP while the RVR and ANP had decreased. In the three ex-PE subgroups, this cyclic pattern with the menstrual cycle was preserved for most of these parameters.

Although baseline hemodynamic and volume status in ex-preeclamptic women differs from that in normal parous controls, its cyclic pattern with the menstrual cycle is largely preserved.

\section{INTRODUCTION}

In healthy women, hemodynamics and volume homeostasis vary with the menstrual cycle ${ }^{12,139}$. The available data provide indirect evidence for mild vasorelaxation in the luteal phase (LP) relative to the follicular phase of the menstrual cycle (FP). In this respect, although much less profound, the LP mimics the early weeks of pregnancy when systemic vasorelaxation is large enough to induce a compensatory rise in cardiac output (CO) together with mild hemodilution as a sign 
of volume expansion and activation of volume regulatory hormones ${ }^{137}$. In contrast, pregnancies complicated by severe fetal growth restriction (IUGR) and/or preeclampsia (PE), are often preceded by defective development of these earlypregnancy changes 5,6 .

Epidemiologic studies indicate that disorders with a thrombophilic phenotype or hypertension predispose to PE ${ }^{64,140}$. Recently, another PE subgroup has been described in which plasma volume (PV) was found to be reduced as compared to normal parous controls ${ }^{141}$. These findings suggest that $\mathrm{PE}$ has different causes. The notion that hemodynamic adaptation in early preeclamptic pregnancy is defective/insufficient raises the question whether hemodynamic and volume cyclicity during the menstrual cycle in ex-preeclamptic women is different from that in women with past uneventful pregnancies.

The objective of the present study was to test the hypothesis that the cyclicity in hemodynamic and volume variables during the menstrual cycle is different in ex$\mathrm{PE}$ women as compared to normal parous controls. To this end, we compared in both phases of the menstrual period blood pressure, cardiac function, renal hemodynamics, PV, neurohumoral status, between ex-PE women and normal parous controls.

\section{MATERIALS AND METHODS}

\section{Selection and patient characteristics}

In this study 39 ovulating ex-preeclamptic non-diabetic caucasian women and 10 normal parous controls were enrolled. Participants were at least 5 months postpartum. Ex-PE women were recruited at the outpatient clinic at the postpartum follow-up, whereas controls were recruited by advertisement. PE and hypertension were defined according to the criteria of the International Society for the Study of Hypertension in Pregnancy (ISSHP) ${ }^{142}$. Therefore, hypertension was defined as a mean systolic and/or a mean diastolic arterial blood pressure higher than $140 \mathrm{mmHg}$ or higher than $90 \mathrm{mmHg}$, respectively, using all of the Dinamap recordings accumulated at the day of the experiment. All experiments were preceded by one week of standardized sodium intake ( $100 \mathrm{mmol}$ sodium per day), representing the mean sodium intake in our population. This required that all participants consulted the hospital's dietitian, prior to study. Women's compliance with the diet was assessed by measuring 24 hour sodium output on the day before the experiment. None of the women used vitamins, oral contraceptives, antihypertensive drugs, NSAID's or glucocorticosteroids in the two weeks prior to the measurements. On separate days and after an overnight fast, participants were tested for thrombophilia and hemodynamic/renal function. All participants gave 
written informed consent. The study was approved by the hospital's medical ethics committee.

\section{Experimental procedure}

Thrombophilic screening. Anticardiolipin antibodies (ACA; IgM and IgG) were determined spectrophotometrically by enzyme-linked immunoassay ${ }^{143}$. Lupus anticoagulant (LA) was determined by mixing 1:1 patient and pooled normal plasma after finding a prolonged partial thromboplastin time. Subsequently, a thromboplastin dilution test was performed by measuring prothrombin time with progressive diluted thromboplastin ${ }^{144}$. LA was considered to be present when all assays were positive. The presence of ACA and/or LA was, when higher than $10 \mathrm{GPL}$ for IgG ACL and 6 MPL for IgM ACL, considered to be indicative of the antiphospholipid syndrome (APS).

Coagulation tests were performed after a flawless venapuncture. Blood was collected in sodium citrate containing glass tubes. Plasma was prepared by two consecutive centrifugation steps, with the second one performed in an Eppendorf centrifuge to eliminate platelets. Samples were stored at $-80^{\circ} \mathrm{C}$ prior to assay. Protein C activity was determined with Coamate protein C (Chromogenix, Mölndal, Sweden) ${ }^{145}$. The normal value of protein $\mathrm{C}$ according to the laboratory control group is $70 \%$ to $130 \%$ and has an intra-assay and inter-assay CV of $3.3 \%$ and $4.7 \%$, respectively. Total protein $S$ antigen and free protein $S$ were measured by an enzyme- linked immunosorbent assay (ELISA) (DAKO, Glostrup, Denmark) 146 . The normal range of both total protein $S$ antigen (intra-assay and inter-assay $\mathrm{CV}$ of $5.1 \%$ and $5.7 \%$ ) and free protein S (intra-assay and inter-assay CV 3.4\% and $3.6 \%$ ) is $70 \%$ to $130 \%$ and $65 \%$ to $130 \%$, respectively. Activated protein C resistance (APCR) was quantified by a chromogenic APC-resistance assay ${ }^{147}$. The chromogenic test fully matches DNA analysis for the factor V Leiden mutation and has a normal range between $0 \%$ and $20 \%{ }^{148}$. Antithrombin III activity (ATIII) was assayed with chromogenic substrate S-2765 (Chromogenix, Mölndal, Sweden) ${ }^{149}$. The normal range of ATIII is $80 \%$ to $120 \%$.

A methionine loading test was used to diagnose hyperhomocysteinemia (HHC). Homocysteine was measured by high-performance liquid chromatography with fluorescence detection ${ }^{150}$. In our hands, this test has an intra- and inter-assay coefficient of variation (CV) of $3.9 \%$ and $5.9 \%$, respectively. At 8:30 AM, the fasting homocysteine concentration was determined. Six hours after an oral dose of L-methionine $(0.1 \mathrm{~g} / \mathrm{kg}$ body weight $)$, the postload homocysteine concentration was assessed. During the test participants used a standard low-methionine breakfast and lunch. Individuals were considered to have HHC when fasting and/or post-load plasma homocysteine levels were more than 2 standard deviations (SD) higher than the mean of the laboratory control group. 
Measurement of blood pressure and renal hemodynamics. Both in the FP (day $5 \pm 2$ ) and LP (day $22 \pm 2$ ) of the menstrual cycle (random order), measurements were performed under standardized environmental conditions in a temperaturecontrolled room $\left(25-26^{\circ} \mathrm{C}\right)$ and with as minimal external disturbances as possible. Participants did not drink caffeine- or alcohol-containing beverages and refrained from smoking and eating for at least 10 hours before the experiment. The measurement session started at 8:00 AM, with the insertion of a 20-gauge catheter into a vein of the right forearm, to enable determination of renal function as detailed below. A second catheter was inserted into a vein of the contralateral forearm for sampling of blood. Throughout each measurement session, subjects were in supine position on a comfortable bed. Arterial blood pressure and heart rate (HR) were recorded repeatedly at two minutes interval by a semi-automatic oscillometric device (Dinamap Vital Signs Monitor 1846, Critikon, Tampa, FL, USA), throughout the measurement session. Effective renal plasma flow (ERPF) and glomerular filtration rate (GFR) were measured by continuous infusion of PAH (PAH: para-aminohippurate sodium; MSD, West Point, PA, USA) and inulin (Inutest, Laevosan Gesellschaft, Linz, Austria), respectively ${ }^{84,151}$. PAH and inulin, measured spectophotometrically, had an intra-assay- and inter-assay CV of $4.2 \%$ and $7.3 \%$, and $4.5 \%$ and $8.1 \%$, respectively. Renal hemodynamics were corrected for body surface area (BSA) and expressed in $\mathrm{ml} \cdot \mathrm{min}^{-1} .1 .73 \mathrm{~m}^{-2}$. ERBF, renal vascular resistance (RVR) and filtration fraction (FF) were calculated as follows:

$$
\begin{aligned}
& E R B F=\text { ERPF } / 1 \text {-hematocrit } \\
& R V R=80,000 \times M A P / E R B F \\
& F F \quad=G F R / E R P F
\end{aligned}
$$

At least two hours after the initiation of the PAH/inulin infusion, three blood samples were taken for determination of inulin and $\mathrm{PAH}$. In addition, blood was collected for assay of active plasma renin concentration (APRC), angiotensin II (AngII), aldosterone (Aldo), atrial natriuretic peptide (ANP), sex-hormones and catecholamines. APRC was determined by an immunoradiometric assay technique (Nichols Institute Diagnostics, Wychen, The Netherlands) with an intraassay CV of $2.9 \%$ and an inter-assay CV of $7.6 \%{ }^{113}$. Angiotensin II (AngII) was assayed by radio-immunoassay (RIA) following Phenyl column extraction (Amersham Int, Amersham, UK) with an intra-assay CV of $4.2 \%$ and an inter-assay CV of $7.7 \%{ }^{114}$. Aldosterone was assessed by a solid-phase protein binding RIA (Diagnostic Products Corporation, LA, USA) with an intra-assay CV of $4.3 \%$ and an inter-assay CV of $6.7 \%{ }^{115}$. Atrial natriuretic peptide (ANP) was analyzed by radioimmunoassay (Nichols Institute Diagnostics, Wychen, The Netherlands) with an intra-assay CV of $9 \%$ and inter-assay CV of $12 \%{ }^{116}$. Norepinephrine (intra- 
assay CV 4.5\%, inter-assay CV 8.1\%), epinephrine ( intra-assay CV 4.1\%, interassay CV 8.6\%) and dopamine (intra-assay CV 3.9\%, inter-assay CV 7.1\%) were assessed by HPLC after liquid-liquid extraction and derivatization with a selective fluorescent agent ${ }^{117}$. Serum $17 \beta$-estradiol and progesterone were determined with a commercially available solid phase radioimmunoassay kit (DPC, Los Angeles, Cal , USA). Sensitivity of the latter assays are 0.035 and $0.15 \mathrm{nmol} / \mathrm{l}$, respectively. Ovulation was confirmed when mid-luteal serum progesterone exceeded $15 \mathrm{nmol} / \mathrm{l}^{81}$. Samples were collected on ice and centrifuged at $4^{\circ} \mathrm{C}$ for $10 \mathrm{~min}$ at $1500 \mathrm{~g}$ immediately after sampling. All samples were stored deep frozen $\left(-80^{\circ} \mathrm{C}\right)$ prior to assay.

Measurement of plasma volume (PV). After the procedure to quantify renal function, PV was measured. Plasma volume (PV, intra-assay CV of $4.2 \%$ ) was determined with the iodine 125-labeled albumin ( ${ }^{125} \mathrm{I}-\mathrm{HSA}$ ) indicator dilution method and expressed in ml per kilogram (kg) lean body mass (LBM). The LBM was calculated as specified elsewhere ${ }^{128}$. PV was obtained by dividing the total injected radioactivity by the virtual volume-specific radioactivity at time zero ${ }^{135}$. The latter, which corresponds with the time of bolus injection, was obtained by extrapolation using the specific activity per ml of plasma at 10,20 and 30 minutes after bolus injection.

Echocardiography to assess cardiac function was performed in semi-left lateral position, after completion of the PV measurement and after 5 minutes of rest, using a cross-sectional, phased array echocardiographic Doppler system (HewlettPackard Sonos 2000 and 2500) ${ }^{137}$.

$$
\begin{aligned}
& C O \text { and cardiac index }(C I) \text { were calculated according to the formulas: } \\
& C O=\text { stroke volume } x H R \\
& C I \quad=C O / B S A
\end{aligned}
$$

In this formula, HR was obtained by taking the mean of five consecutive R-R intervals on the ECG. Stroke volume (SV) was calculated by multiplying the aortic velocity integral and the aortic area. Aortic flow was measured across the aortic valves from an apical approach. The average area under the aortic velocity curve (aortic velocity integral) of five consecutive ejections was used to calculate SV. Aortic valve diameter necessary for the calculation of the aortic area, was measured off-line at the orifice during systole using M-mode. Total peripheral vascular resistance (TPR) and TPR index (TPRi) were calculated as follows:

$$
\begin{aligned}
& T P R=80 \times M A P / C O \\
& T P R i=80 \times M A P / C I
\end{aligned}
$$


Left ventricular work (LVW), defined as the energetic expense on blood imposed by the left ventricle per unit of time was calculated using the formula:

$$
L V W=80 \times M A P \times C O
$$

The value used for MAP was obtained during the $\mathrm{CO}$ measurement and was calculated as the mean of three consecutive recordings.

Data analysis. The allocation of an ex-PE woman to either the hypertensive (HT) or the normotensive subgroup was based on whether or not she had hypertension. If not, then she was allocated to the normotensive thrombophilic group (TP) or the normotensive nonthrombophilic group (REST) based on whether she had a thrombophilic disorder or not. Data are presented as mean \pm SD unless otherwise stated. Differences between groups were tested with the Mann-Whitney-U test along with Bonferroni correction. Differences within groups between the FP and LP were evaluated by Wilcoxon matched-pairs signed-ranks test. Correlation, when calculated, between concommitantly measured potentialy related variables, were tested by Spearman's Rank correlation analysis. In the comparisons a p-value less than 0.05 was considered statistically significant.

\section{RESULTS}

On the basis of the criteria mentioned above 10 of the 39 women were assigned to the HT subgroup. Two women in this subgroup had been diagnosed as having hypertension prior to the study. Among these hypertensive subjects, three were found to have a thrombophilic disorder as well, all in conjunction with HHC. Of the remaining 29 (normotensive) ex-PE subjects, $17(44 \%)$ had one $(n=16)$ - or more than one $(n=1)$ thrombophilic disorder(s). They were allocated to the TP subgroup. The remaining $12 \mathrm{ex}-\mathrm{PE}$ subjects had neither hypertension nor thrombophilia. They were allocated to the REST subgroup. The demographic characteristics of all participants subdivided over the various subgroups are listed in Table 1. The subgroups did not differ with respect to age, height, parity and urinary sodium excretion. Ex-PE females in the REST subgroup had a higher BMI then the controls. Obviously, MAP was higher in the HT subgroup as compared to controls.

The specifics on the thrombophilic disorder in the TP subgroup and some HT subjects are listed in Table 2. Among the 39 ex-preeclamptic participants 51\% $(20 / 39)$ had at least one disorder associated with thrombophilia. In the subjects allocated to the TP subgroup, APS and protein S deficiency were most common, followed by HHC, APCR and protein C deficiency. All ex-PE women which were 
Table 1. Demographic data from the 3 subgroups of ex-preeclamptics and the group of normal parous controls.

\begin{tabular}{|c|c|c|c|c|}
\hline & controls & ex-preeclan & & \\
\hline & $(n=10)$ & $\begin{array}{l}\text { TP } \\
(n=17)\end{array}$ & $\begin{array}{l}\text { REST } \\
(n=12)\end{array}$ & $\begin{array}{l}\text { HT } \\
(n=10)\end{array}$ \\
\hline Age (years) & $31 \pm 2$ & $31 \pm 4$ & $29 \pm 3$ & $33 \pm 4$ \\
\hline Height $(\mathrm{cm})$ & $168 \pm 3$ & $166 \pm 7$ & $167 \pm 5$ & $166 \pm 4$ \\
\hline $\mathrm{BMI}\left(\mathrm{kg} \cdot \mathrm{m}^{-2}\right)$ & $22 \pm 2$ & $24 \pm 4$ & $28 \pm 5 \uparrow$ & $22 \pm 3$ \\
\hline Parity (median with range) & $1(1-2)$ & $1(1-3)$ & $1(1-2)$ & $1(1-4)$ \\
\hline $\mathrm{MAP}(\mathrm{mmHg})$ & $83 \pm 8$ & $84 \pm 8$ & $88 \pm 6$ & $107 \pm 6 \uparrow$ \\
\hline Natriuresis (mmol. $24 \mathrm{~h}^{-1}$ ) & $108 \pm 26$ & $97 \pm 37$ & $97 \pm 33$ & $81 \pm 23$ \\
\hline
\end{tabular}

Table 2. Occurrence of the various subtypes of thrombophilic disorders in the thrombophilic and hypertensive subgroups of ex-PE women.

$\begin{array}{lll}\text { Type of thrombophilic disorder } & \text { TP }(n=17) & \text { HT }(n=1 \\ \text { ACALA (Antiphospholipid syndrome) } & 7(41 \%) & - \\ \text { Hyperhomocysteinemia } & 3(18 \%) & 3(30 \%) \\ \text { Protein C deficiency } & 1(6 \%) & - \\ \text { Activated Protein C Resistance } & 1(6 \%) & - \\ \text { Protein S deficiency } & 6(35 \%) & - \\ \text { AT-III deficiency } & - & -\end{array}$

diagnosed as having APS were IgG serotypes. We did not identify women with AT-III deficiency.

Table 3 lists the systemic hemodynamic data measured in this study, with those from the FP being defined as baseline values. Baseline systemic hemodynamics in the TP subgroup were comparable to those in the control group.Baseline MAP, $\mathrm{CO}, \mathrm{CI}$ and LVW were higher in the HT subgroup as compared to controls. In the REST subgroup baseline values of $\mathrm{CO}$ and LVW were higher and PV lower as compared to the control group. PV did not correlate with BMI in any of the subgroups. With respect to the cyclicity, luteal PV was found to have increased relative to value in the FP, both in controls and in all three ex-PE subgroups. The mean rise in $\mathrm{PV}$ of the control group and the three ex-PE subgroups was found to 
Table 3. Hemodynamic data obtained in the FP and LP of the menstrual cycle.

\begin{tabular}{|c|c|c|c|c|c|}
\hline & & \multirow{2}{*}{$\begin{array}{l}\text { controls } \\
(n=10)\end{array}$} & \multicolumn{3}{|c|}{ ex-preeclamptics } \\
\hline & & & $\begin{array}{l}\text { TP } \\
(n=17)\end{array}$ & $\begin{array}{l}\text { REST } \\
(n=12)\end{array}$ & $\begin{array}{l}\text { HT } \\
(n=10)\end{array}$ \\
\hline \multirow[t]{2}{*}{$\operatorname{MAP}(\mathrm{mmHg})$} & FP & $83 \pm 8$ & $84 \pm 8$ & $88 \pm 6$ & $107 \pm 6 \uparrow$ \\
\hline & LP & $82 \pm 5$ & $84 \pm 7$ & $85 \pm 8$ & $106 \pm 11 \uparrow$ \\
\hline \multirow[t]{2}{*}{ HR (bpm) } & FP & $60 \pm 9$ & $62 \pm 10$ & $67 \pm 8$ & $70 \pm 10$ \\
\hline & LP & $65 \pm 10^{*}$ & $65 \pm 8^{*}$ & $67 \pm 8$ & $70 \pm 12$ \\
\hline \multirow[t]{2}{*}{ SV (ml.beat ${ }^{-1}$ ) } & FP & $74 \pm 8$ & $78 \pm 10$ & $72 \pm 12$ & $79 \pm 11$ \\
\hline & LP & $69 \pm 6$ & $80 \pm 11 \uparrow$ & $76 \pm 12$ & $78 \pm 12$ \\
\hline \multirow[t]{2}{*}{$\operatorname{CO}\left(L \cdot \min ^{-2}\right)$} & FP & $4.6 \pm 0.4$ & $5.3 \pm 0.7$ & $5.5 \pm 0.9 \uparrow$ & $5.9 \pm 0.6 \uparrow$ \\
\hline & LP & $4.6 \pm 0.7$ & $5.5 \pm 1.1$ & $5.6 \pm 1.1$ & $6.0 \pm 0.5 \uparrow$ \\
\hline \multirow[t]{2}{*}{$\mathrm{Cl}\left(\mathrm{L} \cdot \mathrm{min}^{-1} \cdot \mathrm{m}^{-2}\right)$} & FP & $2.7 \pm 0.2$ & $3.1 \pm 0.4$ & $3.0 \pm 0.5$ & $3.5 \pm 0.4 \uparrow$ \\
\hline & LP & $2.7 \pm 0.3$ & $3.2 \pm 0.5 \uparrow$ & $3.1 \pm 0.6$ & $3.6 \pm 0.4 \uparrow$ \\
\hline \multirow{2}{*}{$\begin{array}{l}\text { TPR }\left(x 10^{3}\right) \\
\left(\text { dyne.s. } \mathrm{cm}^{-5}\right)\end{array}$} & FP & $1.4 \pm 0.2$ & $1.3 \pm 0.2$ & $1.3 \pm 0.2$ & $1.5 \pm 0.2$ \\
\hline & LP & $1.5 \pm 0.2$ & $1.2 \pm 0.2 \downarrow$ & $1.3 \pm 0.2$ & $1.4 \pm 0.2$ \\
\hline \multirow{2}{*}{$\begin{array}{l}\mathrm{TPR}_{\mathrm{i}}\left(\mathrm{x} 10^{3}\right) \\
\left(\text { dyne.s. } \mathrm{cm}^{-5} \cdot \mathrm{m}^{-2}\right)\end{array}$} & FP & $2.4 \pm 0.4$ & $2.2 \pm 0.4$ & $2.4 \pm 0.4$ & $2.5 \pm 0.3$ \\
\hline & LP & $2.5 \pm 0.2$ & $2.1 \pm 0.4 \downarrow$ & $2.3 \pm 0.3$ & $2.4 \pm 0.3$ \\
\hline \multirow{2}{*}{$\begin{array}{l}\operatorname{LVW}\left(\times 10^{3}\right) \\
\left(\mathrm{mmHg} \cdot \mathrm{L} \cdot \mathrm{min}^{-1}\right)\end{array}$} & FP & $30 \pm 4$ & $35 \pm 7$ & $39 \pm 10 \uparrow$ & $49 \pm 7 \uparrow$ \\
\hline & LP & $31 \pm 6$ & $35 \pm 9$ & $40 \pm 12 \uparrow$ & $50 \pm 6 \uparrow$ \\
\hline \multirow[t]{2}{*}{$P V\left(m l . \mathrm{kg}^{-1}\right)$} & FP & $55 \pm 4$ & $52 \pm 3$ & $45 \pm 4 \downarrow$ & $53 \pm 5$ \\
\hline & LP & $62 \pm 8^{*}$ & $57 \pm 7^{*}$ & $48 \pm 6^{\star} \downarrow$ & $59 \pm 8^{*}$ \\
\hline
\end{tabular}

Data are presented in mean $\pm S D$. An arrow that follows a particular value indicates this variable to be higher $(\uparrow)$ or lower $(\downarrow)$ than the one in the control group in the corresponding phase of the menstrual cycle. The symbol * indicates the LP value in a particular subgroup to be different from that in the FP. 
Table 4. Renal hemodynamics as determined in the FP and LP of the menstrual cycle.

\begin{tabular}{|c|c|c|c|c|c|}
\hline & & \multirow{2}{*}{$\begin{array}{l}\text { controls } \\
(n=10)\end{array}$} & \multicolumn{3}{|c|}{ ex-preeclamptics } \\
\hline & & & $\begin{array}{l}\text { TP } \\
(n=17)\end{array}$ & $\begin{array}{l}\text { REST } \\
(n=12)\end{array}$ & $\begin{array}{l}\text { HT } \\
(n=10)\end{array}$ \\
\hline \multirow{2}{*}{$\begin{array}{l}\text { GFR } \\
\left(\mathrm{ml} \cdot \mathrm{min}^{-1} \cdot 1.73 \mathrm{~m}^{-2}\right)\end{array}$} & FP & $125 \pm 8$ & $115 \pm 13$ & $114 \pm 24$ & $111 \pm 10$ \\
\hline & $\mathrm{LP}$ & $130 \pm 12$ & $119 \pm 18$ & $122 \pm 27^{*}$ & $120 \pm 18^{*}$ \\
\hline \multirow{2}{*}{$\begin{array}{l}\text { ERPF } \\
\qquad\left(\mathrm{ml} \cdot \mathrm{min}^{-1} \cdot 1.73 \mathrm{~m}^{-2}\right)\end{array}$} & FP & $565 \pm 77$ & $495 \pm 71$ & $500 \pm 98$ & $480 \pm 105$ \\
\hline & LP & $596 \pm 64^{*}$ & $516 \pm 77 \downarrow$ & $519 \pm 111$ & $557 \pm 133^{*}$ \\
\hline \multirow{2}{*}{$\begin{array}{l}\text { ERBF } \\
\left(\mathrm{ml} \cdot \mathrm{min}^{-1} \cdot 1.73 \mathrm{~m}^{-2}\right)\end{array}$} & FP & $881 \pm 127$ & $777 \pm 129$ & $791 \pm 144$ & $770 \pm 169$ \\
\hline & LP & $935 \pm 105^{*}$ & $809 \pm 132$ & $820 \pm 163$ & $897 \pm 205^{\star}$ \\
\hline \multirow{2}{*}{$\begin{array}{l}\operatorname{RVR}\left(x 10^{3}\right) \\
\left(\text { dyn.s. } \mathrm{cm}^{-5} \cdot 1.73 \mathrm{~m}^{-2}\right)\end{array}$} & FP & $7.2 \pm 1.3$ & $8.4 \pm 1.6 \uparrow$ & $8.6 \pm 1.5 \uparrow$ & $10.9 \pm 2.6 \uparrow$ \\
\hline & $\mathrm{LP}$ & $6.7 \pm 0.9^{*}$ & $7.9 \pm 1.5$ & $8.2 \pm 1.7$ & $9.2 \pm 2.6 * \uparrow$ \\
\hline \multirow{2}{*}{$\begin{array}{l}\text { FF } \\
\text { (GFR/ERPF) }\end{array}$} & FP & $0.22 \pm 0.03$ & $0.23 \pm 0.03$ & $0.23 \pm 0.02$ & $0.24 \pm 0.04$ \\
\hline & LP & $0.22 \pm 0.03$ & $0.23 \pm 0.03$ & $0.24 \pm 0.03$ & $0.22 \pm 0.05^{*}$ \\
\hline \multirow{2}{*}{$\begin{array}{l}\text { Sodium excr. } \\
\left(\mathrm{mmol}^{2} 24 \mathrm{~h}^{-1}\right)\end{array}$} & FP & $108 \pm 26$ & $97 \pm 37$ & $97 \pm 33$ & $81 \pm 23$ \\
\hline & LP & $123 \pm 32$ & $89 \pm 39 \downarrow$ & $92 \pm 25$ & $84 \pm 30 \downarrow$ \\
\hline
\end{tabular}

Data are presented in mean \pm SD. An arrow that follows a particular value indicates this variable to be higher $(\uparrow)$ or lower $(\downarrow)$ than the one in the control group in the corresponding phase of the menstrual cycle. The symbol * indicates the LP value in a particular subgroup to be different from that in the FP.

be comparable. Luteal HR was consistently higher in the controls and in the TP subgroup, but not in the REST - and HT subgroups. In neither group did any of the other hemodynamic variables listed in Table 3 vary with the menstrual cycle. The renal hemodynamic data are listed in Table 4. All baseline data points were comparable between groups except for a higher RVR in all ex-PE subgroups relative to the controls. The cyclicity in renal hemodynamics differed between groups. In the control group luteal ERPF and ERBF had increased and the RVR had decreased relative to the FP. In the HT subgroup these changes were supplemented with a luteal rise in GFR and fall in FF relative to the FP. In the REST subgroup only the luteal rise in GFR reached significance whereas in the TP subgroup none of the renal variables had changed significantly between the FP and LP. In the HT 
Table 5. Endocrine variables as determined in the FP and LP of the menstrual cycle.

\begin{tabular}{|c|c|c|c|c|c|}
\hline & & controls & ex-preeclampt & & \\
\hline & & $(n=10)$ & $\begin{array}{l}\text { TP } \\
(n=17)\end{array}$ & $\begin{array}{l}\text { REST } \\
(n=12)\end{array}$ & $\begin{array}{l}\text { HT } \\
(n=10)\end{array}$ \\
\hline APRC & FP & $13.7 \pm 4.5$ & $12.1 \pm 6.9$ & $14.0 \pm 5.3$ & $18.3 \pm 9.4$ \\
\hline$\left(m U . L^{-1}\right)$ & LP & $21.7 \pm 7.3^{*}$ & $23.7 \pm 11.6^{*}$ & $21.2 \pm 5.5^{*}$ & $25.9 \pm 10.4^{*}$ \\
\hline Angll & FP & $8.7 \pm 2.4$ & $9.9 \pm 3.7$ & $10.2 \pm 2.4$ & $10.7 \pm 3.7$ \\
\hline$\left(\right.$ pmol. $\left.L^{-1}\right)$ & LP & $13.7 \pm 10.4^{*}$ & $12.9 \pm 5.1^{*}$ & $13.0 \pm 4.3^{*}$ & $15.7 \pm 6.0^{*}$ \\
\hline Aldo & FP & $85 \pm 30$ & $82 \pm 23$ & $107 \pm 39$ & $109 \pm 68$ \\
\hline$\left(\mathrm{nmol} . \mathrm{L}^{-1}\right)$ & LP & $203 \pm 141^{*}$ & $233 \pm 106^{*}$ & $285 \pm 175^{\star}$ & $183 \pm 105^{\star}$ \\
\hline ANP & FP & $93 \pm 26$ & $97 \pm 34$ & $105 \pm 50$ & $141 \pm 46 \uparrow$ \\
\hline (ng . $L^{-1}$ ) & LP & $84 \pm 21^{*}$ & $77 \pm 29 *$ & $98 \pm 31$ & $126 \pm 73^{*}$ \\
\hline Norepinephrine & FP & $0.63 \pm 0.20$ & $0.84 \pm 0.47$ & $0.86 \pm 0.19$ & $1.28 \pm 0.64 \uparrow$ \\
\hline$\left(\mathrm{nmol} . \mathrm{L}^{-1}\right)$ & LP & $1.02 \pm 0.20^{*}$ & $1.25 \pm 0.68 *$ & $1.01 \pm 0.24^{*}$ & $1.27 \pm 0.85$ \\
\hline Epinephrine & FP & $0.06 \pm 0.04$ & $0.06 \pm 0.03$ & $0.06 \pm 0.03$ & $0.10 \pm 0.09$ \\
\hline$\left(\mathrm{nmol} . \mathrm{L}^{-1}\right)$ & LP & $0.05 \pm 0.03$ & $0.06 \pm 0.03$ & $0.07 \pm 0.07$ & $0.06 \pm 0.05$ \\
\hline Dopamine & FP & $0.21 \pm 0.12$ & $0.27 \pm 0.13$ & $0.18 \pm 0.07$ & $0.18 \pm 0.09$ \\
\hline$\left(\mathrm{nmol} . \mathrm{L}^{-1}\right)$ & LP & $0.21 \pm 0.10$ & $0.22 \pm 0.11$ & $0.23 \pm 0.10$ & $0.19 \pm 0.10$ \\
\hline $17 \beta$-Estradiol & FP & $0.13 \pm 0.09$ & $0.14 \pm 0.05$ & $0.15 \pm 0.04$ & $0.16 \pm 0.07$ \\
\hline (nmol. $\mathrm{L}^{-1}$ ) & LP & $0.25 \pm 0.11^{*}$ & $0.34 \pm 0.12 *$ & $0.31 \pm 0.10 *$ & $0.35 \pm 0.11$ * \\
\hline Progesterone & FP & $1.3 \pm 0.5$ & $2.2 \pm 1.1$ & $2.3 \pm 1.4$ & $1.7 \pm 0.8$ \\
\hline$\left(\mathrm{nmol} . \mathrm{L}^{-1}\right)$ & LP & $30.1 \pm 14.2^{*}$ & $29.7 \pm 9.2^{*}$ & $26.5 \pm 9.6^{*}$ & $29.6 \pm 10.9$ * \\
\hline
\end{tabular}

Data are presented in mean $\pm S D$. An arrow that follows a particular value indicates this variable to be higher $(\uparrow)$ or lower $(\downarrow)$ than the one in the control group in the corresponding phase of the menstrual cycle. The symbol * indicates the LP value in a particular subgroup to be different from that in the FP. 
and TP subgroups, the luteal natriuresis was lower than that in the FP, most likely due to less compliance with the diet.

The endocrine data are listed in Table 5. Baseline ANP and norepinephrine were higher in the HT subgroup then in the control group. With respect to the cyclicity we noted a luteal rise in the hormones of the renin-angiotensin-aldosterone (RAA) system and a fall in ANP in all groups except for REST women. Except for the HT subgroup, in all other groups luteal norepinephrine was found to have increased relative to the FP. The difference between the luteal and follicular value for the other neurohormones was inconsistent. Finally, baseline levels and cyclic change in $17 \beta$-estradiol and progesterone were comparable in all groups.

\section{DISCUSSION}

Endothelial dysfunction plays a central role in the development of PE ${ }^{152}$. Endothelial function can be jeopardized either biochemically (i.e. thrombophilia, diabetes mellitus) or mechanically (i.e. hypertension, renovascular disease) ${ }^{140,153}$. PE seems to be superimposed upon a preexisting disorder and may be the clinical expression of "endothelial decompensation" which represents the common endpoint of these two different pathophysiologic pathways. We therefore subdivided our group of ex-PE subjects into a TP and HT subgroup, thus separating them from a group of normotensive ex-PE women without thrombophilia (REST). Since in healthy females menstrual changes in hemodynamics and renal function largely mimic early pregnancy changes and $\mathrm{PE}$ often is preceded by hemodynamic and renal maladaptation, these finding raised the question whether cyclic menstrual changes in these functions, as seen in healthy women, would be preserved in the above mentioned ex-PE subgroups. To answer this question, we studied in the follicular phase and the luteal phase of the menstrual cycle, clotting, metabolic, renal and cardiovascular function in ex-preeclamptic women and controls and compared the data in the various ex-PE subgroups with those found in a reference group of normal parous controls.

With respect to the general characteristics, the three subgroups were comparable both with each other and with the control group, except for a higher BMI in the REST subgroup. In most women in the TP subgroup, thrombophilia was caused by AFS, protein $S$ deficiency and HHC, an observation in line with reports by others ${ }^{64}$. Comparison of all baseline hemodynamic, renal and endocrine data in the three ex-PE subgroups relative to the control group indicated that in the HT subgroup, the higher MAP was paralleled by a higher CO and CI, but a comparable TPR at the expence of a higher LVW. In this group renovascular resistance was increased and circulating levels of ANP and norepinephrine elevated. It is unlikely that the higher CI was secondary to the awareness of having hypertension as in 
only 2 of the 10 women in the HT subgroup the diagnosis "hypertension" was made at the time of the study and the raised blood pressure was seen during both measurement sessions ${ }^{154}$. Most baseline values for central and renal hemodynamics and endocrine status in the TP subgroup resembled those in the control group. This indicates that the altered endothelial function in response to thrombophilia does not seem to influence appreciably cardiovascular function. In the REST subgroup the relative overweight and the lower PV were most conspicious.

With respect to the central and renal hemodynamic cyclicity during the menstrual cycle, we found a rise in luteal HR, ERPF and PV in the control group. RVR fell during the LP in this group. These changes were accompanied by activation of the RAA-system, a rise in plasma norepinephrine and a fall in ANP. These changes are in agreement with observations by others ${ }^{12,139,155}$ and are compatible with a state of vasorelaxation giving rise to volume expansion as a result of activation of the sympathetic system and the RAA-system with adaptive suppression of ANP. In contrast to controls, the HT subgroup had higher basal circulating norepinephrine levels which did not fluctuate with the menstrual cycle. Otherwise, their cyclic changes in renal and endocrine variables were comparable to those in controls. In the normotensive ex-PE subgroups renal hemodynamics did not change significantly with the menstrual cycle. Hormonal and plasma volume changes, however, were similar as in controls. These observations indicate that most hemodynamic, renal and neuroendocrine changes with the menstrual cycle are preserved in ex-PE individuals.

Although it is known, that in the early stages of essential hypertension PV is reduced ${ }^{156}$, we did not find such an abnormality in our hypertensive ex-PE patients but we did observe a reduced PV in the REST subgroup of ex-PE patients. The latter, therefore, suggests a condition of 'borderline hypertension' in these patients. The lower PV could then be viewed upon as a compensatory mechanism to minimize a rise in pressure. Why PV is not reduced in the HT subgroup, is less easy to explain. Perhaps this group already has more advanced renal lesions which interfere with volume control.

In summary, although baseline hemodynamics and volume status differed between ex-PE subgroups and controls, the cyclicity with the menstrual cycle in the three subgroups of ex-PE women, was preserved. 



\section{General discussion}

Epidemiological studies suggest a multifactorial pathogenesis of preeclampsia ${ }^{140}$. Besides immune maladaptation and genetic predisposition, various vascular factors have been identified that enhance the development of preeclampsia. Irrespective of its primary cause, however, there is general agreement that the first demonstrable abnormality in preeclamptic pregnancy is defective throphoblast invasion giving rise to poor placentation ${ }^{32,157}$. Subsequently, the malfunctioning placenta may cause intra-uterine growth retardation (IUGR) in the second half of pregnancy. In some women the clinical expression of poor placentation is preeclampsia rather than IUGR. The mechanism by which the poorly developed placenta triggers preeclampsia is still unsettled. It has been suggested that the malfunctioning placenta releases some "factor X" which leads to endothelial dysfunction and with it, most of the symptoms of preeclampsia ${ }^{70}$. However, convincing experimental proof for this concept is lacking. In the present thesis we set out to study hemodynamic and volume regulating factors as a possible cause of preeclampsia.

Several years ago, we observed already that in the $5^{\text {th }}$ week of supposedly normal pregnancy cardiac output $(\mathrm{CO})$ rises, which is associated with a fall in blood pressure and hemodilution ${ }^{112}$. These changes are consistent with maternal systemic vasorelaxation and relative hypovolemia and are followed by a steady increase in the ultrasonic dimensions of the left atrium and inferior vena cava ${ }^{112}$. These observations led us to postulate the following sequence of events in normal human pregnancy. Within two weeks after implantation, some still unknown pregnancyspecific mechanism induces maternal systemic vasorelaxation giving rise to a high-flow/low-resistence circulation. The extra blood volume needed to maintain such a circulation for a prolonged period of time is met by enhanced volume retention ${ }^{158}$.

Some of the seemingly healthy participants in our study developed IUGR and/or preeclampsia later on in their pregnancy. Further analysis revealed that, at the start of the study in early pregnancy, these seemingly healthy subjects had smaller central cardiovascular dimensions as compared to the subjects with uneventful pregnancy ${ }^{112}$. In addition, they failed to respond to pregnancy with systemic vasorelaxation and plasma volume (PV) expansion. The sequence of events in these pregnancies may thus be as follows. A preexisting subnormal filling of the vascular bed is related to enhanced vasoconstriction in these women. Any vasore- 
laxing stimulus from pregnancy must now be offset by enhanced sympathetic stimulation and activation of the RAA-system as long as these women are unable to provide adequate filling of their vasculature. In this respect, the recently observed state of sympathetic overactivity in preeclamptics is consistent with this concept ${ }^{138}$. The proposed hemodynamic maladaptation in early pregnancy may interfere with the decidual perfusion at the implantation site causing deficient trophoblast invasion and with it defective placental development.

In recent years various research groups have studied hemodynamic patterns during the menstrual cycle. The results were conflicting although there was indirect evidence for mild vasorelaxation in the luteal phase resembling that in early pregnancy ${ }^{7,11}$. These observations formed the basis for the set of studies in the present thesis. The overall hypothesis we intended to test was:

In women with preeclamptic pregnancy, maladaptation of central and renal hemodynamics in early pregnancy is reflected in abnormal hemodynamic and volume regulatory changes in the luteal phase (LP) relative to the follicular phase (FP) of the menstrual cycle.

To test this hypothesis we first studied hemodynamic and volume regulatory cyclicity in healthy nulliparous women (chapter 3, 4 and 5), to obtain reference values. We also tried to determine the individual contribution of $17 \beta$-estradiol and progesterone to the observed cyclic changes in hemodynamics and volume homeostasis (chapter 6). Finally, we studied hemodynamics, renal function and body fluid volumes under basal conditions (chapter 7) and during the menstrual cycle (chapter 8 ) in women with a history of preeclampsia.

In a pilot study ${ }^{12}$ in nine women with an ovulatory menstrual cycle, we could not substantiate the finding of generalized vasorelaxation during the LP of the menstrual cycle. In that study we employed non-invasive techniques to measure venous and arterial tone, glomerular filtation rate (GFR) and blood flows to skin, forearm and kidneys. Luteal skin blood flow was consistently lower and luteal GFR higher than values obtained in the FP. However, none of the other variables changed with the menstrual cycle. The observed luteal fall in arteriovenous shunt flow to the skin was considered functional as it raises core temperature, a response which can be expected in conjunction with the well-known progesteronedependent upward resetting of the thermoregulatory center. The $10 \%$ higher GFR together with a trend towards a lower renal vascular resistance (RVR) in the LP suggest postovulatory renal vasodilation. Because of the small population sample and the large measurement error for most of the variables studied, the power of this study was modest. However, renal hemodynamics are influenced by dietary 
sodium, a factor which was not controlled for in the present study. We also thought that information on cardiac output and body fluid volumes could be necessary to describe (systemic) vascular changes with the menstrual cycle. It follows that the results of our pilot study were inadequate to accept or reject our initial hypothesis on the occurrence of systemic vasorelaxation in the LP of the menstrual cycle.

Thus, we decided to perform a similar study in a larger population with the participating subjects using a standardized sodium intake at the time of study. This time we measured CO, PV, extracellular fluid volume (ECFV) and the circulating levels of hormones involved in volume regulation. We calculated both for the FP and the LP, total peripheral vascular resistance (TPR), RVR and forearm vascular resistance (FVR). Because of the effect of salt intake on the latter variables, we performed our measurements in two consecutive menstrual cycles with the subjects using in random order, a low- and a high-salt diet. In spite of a regular menstrual cycle ( $29 \pm 3$ days) in all subjects enrolled, only $61 \%$ and $44 \%$ of the high-salt and low-salt menstrual cycles, respectively, were ovulatory according to our criterium of a mid-luteal circulating serum progesterone level above $15 \mathrm{nmol} / \mathrm{l}$. We do not have an explanation for these relatively low percentages, except chance. In our pilot study $90 \%$ of the menstrual cycles were ovulatory.

Salt intake clearly modified hemodynamic and volume regulatory changes with the menstrual cycle (Chapter 4 and 5), as consistent changes were only observed during high-salt diet. On a low-salt diet the cyclicity in the measured variables was abolished. Compliance with the diet was satisfactory as during high-salt diet, relative to low-salt diet, baseline values of body weight, ECFV and plasma concentration of atrial natriuretic peptide (ANP) were higher while RAA-system activity decreased. The hemodynamic, renal and fluid volume changes with the menstrual cycle during a high-salt diet consisted of a lower luteal TPR, filtration fraction (FF) and RVR and a higher luteal effective renal plama flow (ERPF) and GFR. Changes in FVR, ECFV and PV were inconsistent. The changes in volume regulatory hormones with the menstrual cycle during high-salt intake consisted of a higher luteal RAA-system activity together with a trend towards a lower luteal ANP. Part of these findings have recently been confirmed by another group ${ }^{109}$. The results of our study can be summarized as follows. The pattern of hemodynamic changes in the LP of the menstrual cycle consists of a mild systemic vasorelaxation, which is also demonstrable in the kidney, and with the amplitude increasing as a function of salt intake. The endocrine changes in the LP suggest activated sodium retention, but the lack of an increase in PV indicates that this is not very pronounced. The disappearance of hemodynamic/renal cyclicity during low-salt intake is interesting. It suggests that a certain amount of salt intake is 
needed for the vasculature to relax in the LP. In other words, vasorelaxation can occur only in the face of a sufficiently expanded ECFV. The hemodynamic, renal and volume regulatory changes in LP differed from those in early pregnancy by a lack of increase in skin blood flow, muscle blood flow and PV. Moreover, hemodilution was not observed. In addition, renal vasodilation in the LP was less pronounced and the luteal rise in RAA-system activity and fall in TPR were small when compared with changes of these variables in early pregnancy. By extrapolating these findings to early pregnancy, one could speculate about the need for sufficient sodium intake, particularly during this period of hemodynamic adaptation. In this light, the advice to restrict salt intake would do more harm than good.

The relatively high percentage of anovulatory cycles allowed us to compare these cycles with ovulatory cycles, thus providing some insight in the individual roles of progesterone and $17 \beta$-estradiol in the hemodynamic changes during the menstrual cycle (Chapter 6 ). In view of the above, only high-salt menstrual cycles were included in this analysis. The luteal rise in serum $17 \beta$-estradiol was comparable in anovulatory and ovulatory cycles. By definition, the luteal rise in serum progesterone was much larger in the ovulatory cycles. The cyclic change in TPR, GFR, RAA-system activity and ANP were comparable in both types of cycles, suggesting a primary role for $17 \beta$-estradiol in these changes. On the other hand, only in ovulatory cycles we found a luteal increase in ERPF in concert with a fall in FF. The latter supports the view that progesterone induces selective relaxation of the efferent renal arterioles, an observation in line with previous pharmacological studies by others ${ }^{122}$. It follows that the well-known natriuretic effect of progesterone may be accomplished not only by its affinity for the aldosterone receptor, but also by reducing the $\mathrm{FF}^{122}$. In this concept, a lower $\mathrm{FF}$ would lead to a lower peritubular oncotic pressure, which can be expected to reduce proximal tubular sodium and water reabsorption ${ }^{159}$. There is some indirect evidence from an in-vitro study that progesterone may induce selective vasodilation of the efferent renal arterioles by down-regulation of AT1 receptors in these vessels ${ }^{129}$. However, the exploration of the underlying mechanism of this particular progesterone effect, requires further investigation.

The results of our studies into the menstrual cycle of healthy women can now be summarized as follows. The LP of the menstrual cycle is characterized by mild salt-dependent vasodilation in the kidney. This effect is paralleled by a higher RAA-system activity and lower circulating levels of ANP, but without an appreciable change in PV and ECFV. It follows that changes in these fluid compartments are small. The observed cyclic changes may be triggered by $17 \beta$-estradiolmediated mild generalized vasorelaxation and progesterone-mediated selective vasodilation of the efferent arterioles in the kidneys. At first sight, these changes 
resemble those in early pregnancy. However, the spectrum of changes in early pregnancy is much wider and their magnitude much greater than those in the LP. Therefore, the luteal change in hemodynamics differs from that in early pregnancy in a quantitative way.

Apparently, healthy women with a history of preeclampsia and/or IUGR have an increased risk to develop preeclampsia in a subsequent pregnancy ${ }^{160}$. In these women, the prevalence of latent abnormalities in e.g. clotting function, metabolism and the immune system is much higher than in a reference group of normal parous controls ${ }^{64}$. Since hypertension is also preceded by a long latent period with subtle abnormalities in renal hemodynamics and the RAA-system ${ }^{133}$, we decided to determine whether basal hemodynamics in the FP and hemodynamic changes with the menstrual cycle in women with a recent history of preeclampsia differ from those in parous women with a history of only uneventful pregnancies (chapter 7). In this particular study we tested the hypothesis that asymptomatic and seemingly healthy women with a history of pre-eclampsia do have abnormalities in their renal hemodynamics and volume status. Although RAA-system activity in ex-preeclamptics did not differ from that in controls, ERPF was lower, and FF and RVR were higher in ex-preeclamptics than in controls. The normotensive subgroup of ex-preeclamptics had a $10 \%$ lower PV, and a more than $50 \%$ lower ANP as compared to controls. These findings support the hypothesis that also seemingly healthy women with a history of preeclampsia have abnormalities in their volume status and renal hemodynamics. If these changes would already be present prior to a pregnancy complicated by preeclampsia, it is tempting to speculate that there is a particular subgroup of seemingly healthy women with some (sub)clinical vascular disease that has relative hypovolemia as common denominator.

Finally, we compared the fluctuations in hemodynamic, renal and volume regulatory functions with the menstrual cycle in ex-preeclamptics with those in normal parous controls (Chapter 8). Seemingly healthy ex-preeclamptics were more obese and had a lower basal PV than parous controls. Meanwhile, the cyclicity in all variables studied, including PV, did not differ between the ex-preeclamptics and controls. In this study, luteal PV was higher than follicular PV in both the ex-preeclamptics and the controls. In our previous study PV did not vary with the menstrual cycle in the approx. 10 years younger nulliparous participants. Possibly, a higher age and/or multiparity increase the amplitude of the cyclic fluctuations in $\mathrm{PV}$ to above detection level. This stresses the importance of adequate matching for parity, age and salt intake in this type of studies.

In conclusion, in both healthy and ex-preeclamptic women, hemodynamic, renal and volume regulatory functions do vary with the menstrual cycle, provided an 
adequate amount of salt is consumed. As compared to early pregnancy, the number of variables that change in the LP is much smaller, and the magnitude of the observed changes is always modest, often barely above detection level. Therefore, we reject our overall hypothesis that maladaptation in early pregnancy is reflected in abnormal hemodynamic and volume regulatory changes with the menstrual cycle. $17 \beta$-estradiol and progesterone act in concert to induce the observed changes with the menstrual cycle. The magnified changes in early pregnancy are probably dependent on the presence of an additional pregnancy-specific vasorelaxing factor. On the other hand basal hemodynamic, renal or volume regulatory variables may differ between ex-preeclamptics and controls. Seemingly healthy ex-preeclamptics without latent disorders such as thrombophilia, the antiphospholipid syndrome or hyperhomocysteinemia, are more obese and tend to have a reduced PV. It is possible that a reduced PV in women at risk for preeclampsia initiates the cascade of events that leads to poor placentation. On the other hand, it is also conceivable that the reduced PV is only a confounder of some other latent hemodynamic disorder. Hemodynamics and volume regulation in early pregnancy rather than during the menstrual cycle, should be subject to future study. The objectives of such prospective studies should be to explore the mechanism responsible for the early-pregnancy vasorelaxation, and to assess to what extent the hypovolemic state in ex-preeclamptics contributes to the pathogenesis of preeclampsia. 


\section{References}

1. National High Blood Pressure Education Program Working Group Report on High Blood Pressure in Pregnancy. Am J Obstet Gynecol 1990; 163:1691-1712.

2. Berg CJ, Atrash HK, Koonin LM, Tucker M. Pregnancy-related mortality in the United States, 1987-1990. Obstet Gynecol 1996; 88:161-167.

3 Schuitemaker NWE, Bennebroek Gravenhorst J, Dekker GA, van Dongen PWJ, van Geijn HP. Moedersterfte in Nederland 1988-1992. Nederlands Tijdschrift voor Obstetrie en Gynaecologie 1993; 106:270-271.

4. Redman CW, Roberts JM. Management of pre-eclampsia. Lancet 1993; 341: 14511454.

5. Duvekot JJ, Cheriex EC, Pieters FA, Peeters LLH. Severely impaired fetal growth is preceded by maternal hemodynamic maladaptation in very early pregnancy. Acta Obstet Gynecol Scand 1995; 74:693-697.

6. Duvekot JJ, Cheriex EC, Pieters FA, Menheere PP, Schouten HJ, Peeters LLH. Maternal volume homeostasis in early pregnancy in relation to fetal growth restriction. Obstet Gynecol 1995; 85:361-367.

7. Dunne FP, Barry DG, Ferriss JB, Grealy G, Murphy D. Changes in blood pressure during the normal menstrual cycle. Clin Sci Colch 1991; 81:515-518.

8. Vokes TJ, Weiss NM, Schreiber J, Gaskill MB, Robertson GL. Osmoregulation of thirst and vasopressin during normal menstrual cycle. Am J Physiol 1988; 254:R641-R647.

9. Davison JM, Noble MC. Serial changes in 24 hour creatinine clearance during normal menstrual cycles and the first trimester of pregnancy. Br J Obstet Gynaecol 1981; 88:10-17.

10. Paaby P, Moller Petersen J, Larsen CE, Raffn K. Endogenous overnight creatinine clearance, serum beta 2-microglobulin and serum water during the menstrual cycle. Acta Med Scand 1987; 221:191-197.

11. Brochner Mortensen J, Paaby P, Fjeldborg P, Raffn K, Larsen CE, Moller Petersen J. Renal haemodynamics and extracellular homeostasis during the menstrual cycle. Scand J Clin Lab Invest 1987; 47:829-835.

12. van Beek E, Houben AJHM, van Es PN, et al. Peripheral haemodynamics and renal function in relation to the menstrual cycle. Clin Sci 1996; 91:163-168.

13. Brown JJ, Davies DL, Lever AF, Robertson JIS. Variations in plasma renin during the menstrual cycle. Br Med J 1964; 2:1114-1115.

14. Katz FH, Romfh P. Plasma aldosterone and renin activity during the menstrual cycle. J Clin Endocrinol Metab 1972; 34:819-821. 
15. Sealey JE, Itskovitz Eldor J, Rubattu S, et al. Estradiol- and progesterone-related increases in the renin-aldosterone system: studies during ovarian stimulation and early pregnancy. J Clin Endocrinol Metab 1994; 79:258-264.

16. Spargo BH, Lichtig C, Luger AM, Katz AI, Lindheimer MD. The renal lesion in preeclampsia. In: Lindheimer MD, Katz AI, Zuspan FP, eds. Hypertension in pregnancy. New York: John Wiley \& Sons, Inc., 1976:129-137.

17. Shanklin DR, Sibai BM. Ultrastructural aspects of preeclampsia. I. Placental bed and uterine boundary vessels. Am J Obstet Gynecol 1989; 161:735-741.

18. Redman CW, Denson KW, Beilin LJ, Bolton FG, Stirrat GM. Factor-VIII consumption in pre-eclampsia. Lancet 1977; 2:1249-1252.

19. Inglis TC, Stuart J, George AJ, Davies AJ. Haemostatic and rheological changes in normal pregnancy and pre-eclampsia. Br J Haematol 1982; 50:461-465.

20. Kraayenbrink AA, Dekker GA, van Kamp GJ, van Geijn HP. Endothelial vasoactive mediators in preeclampsia. Am J Obstet Gynecol 1993; 169:160-165.

21. Florijn KW, Derkx FH, Visser W, et al. Elevated plasma levels of endothelin in preeclampsia. J Hypertens Suppl 1991; 9:S166-S167.

22. Lazarchick J, Stubbs TM, Romein L, Van Dorsten JP, Loadholt CB. Predictive value of fibronectin levels in normotensive gravid women destined to become preeclamptic. Am J Obstet Gynecol 1986; 154:1050-1052.

23. Ballegeer V, Spitz B, Kieckens L, Moreau H, Van Assche A, Collen D. Predictive value of increased plasma levels of fibronectin in gestational hypertension. Am J Obstet Gynecol 1989; 161:432-436.

24. Lockwood CJ, Peters JH. Increased plasma levels of ED1+ cellular fibronectin precede the clinical signs of preeclampsia. Am J Obstet Gynecol 1990; 162:358-362.

25. de Boer K, Lecander I, ten Cate JW, Borm JJ, Treffers PE. Placental-type plasminogen activator inhibitor in preeclampsia. Am J Obstet Gynecol 1988; 158:518-522.

26. Walsh SW. Preeclampsia: an imbalance in placental prostacyclin and thromboxane production. Am J Obstet Gynecol 1985; 152:335-340.

27. Redman CW. Current topic: pre-eclampsia and the placenta. Placenta 1991; 12:301-308.

28. Roberts JM, Taylor RN, Musci TJ, Rodgers GM, Hubel CA, McLaughlin MK. Preeclampsia: an endothelial cell disorder. Am J Obstet Gynecol 1989; 161:12001204.

29. Piering WF, Garancis JG, Becker CG, Beres JA, Lemann JJ. Preeclampsia related to a functioning extrauterine placenta: report of a case and 25-year follow-up. Am J Kidney Dis 1993; 21:310-313.

30. Brosens IA, Robertson WB, Dixon HG. The role of the spiral arteries in the pathogenesis of preeclampsia. Obstet Gynecol Annu 1972; 1:177-191.

31. Pijnenborg R. The placental bed. Hypertens Pregnancy 1996; 15 (1):7-23.

32. Khong TY, De Wolf F, Robertson WB, Brosens I. Inadequate maternal vascular response to placentation in pregnancies complicated by pre-eclampsia and by small-forgestational age infants. Br J Obstet Gynaecol 1986; 93:1049-1059. 
33. Roberts JM, Redman CW. Pre-eclampsia: more than pregnancy-induced hypertension. Lancet 1993; 341:1447-1451.

34. Kitzmiller JL, Benirschke K. Immunofluorescent study of placental bed vessels in preeclampsia of pregnancy. Am J Obstet Gynecol 1973; 115:248-251.

35. Fox H. The placenta in pregnancy hypertension. In: Rubin PC, ed. Handbook of hypertension, volume 10. Hypertension in pregnancy. Amsterdam: Elsevier Science Publisher B.V., 1988:16-37.

36. Combs CA, Katz MA, Kitzmiller JL, Brescia RJ. Experimental preeclampsia produced by chronic constriction of the lower aorta: validation with longitudinal blood pressure measurements in conscious rhesus monkeys. Am J Obstet Gynecol 1993; 169:215-223.

37. Feeney JG, Scott JS. Pre-eclampsia and changed paternity. Eur J Obstet Gynecol Reprod Biol 1980; 11:35-38.

38. Campbell DM, MacGillivray I, Carr Hill R. Pre-eclampsia in second pregnancy. Br J Obstet Gynaecol 1985; 92:131-140.

39. Strickland DM, Guzick DS, Cox K, Gant NF, Rosenfeld CR. The relationship between abortion in the first pregnancy and development of pregnancy-induced hypertension in the subsequent pregnancy. Am J Obstet Gynecol 1986; 154:146-148.

40. Feeney JG, Tovey LA, Scott JS. Influence of previous blood-transfusion on incidence of pre-eclampsia. Lancet 1977; 1:874-875.

41. Marti JJ, Herrmann U. Immunogestosis: a new etiologic concept of "essential" EPH gestosis, with special consideration of the primigravid patient; preliminary report of a clinical study. Am J Obstet Gynecol 1977; 128:489-493.

42. Robillard PY, Hulsey TC, Perianin J, Janky E, Miri EH, Papiernik E. Association of pregnancy-induced hypertension with duration of sexual cohabitation before conception. Lancet 1994; 344:973-975.

43. Klonoff Cohen HS, Savitz DA, Cefalo RC, McCann MF. An epidemiologic study of contraception and preeclampsia. JAMA 1989; 262:3143-3147.

44. Serhal PF, Craft I. Immune basis for pre-eclampsia evidence from oocyte recipients. Lancet $1987 ; 2: 744$.

45. El-Roeiy A, Gleicher N. The immunologic concept of pre-eclampsia. In: Rubin PC, ed. Handbook of hypertension, volume 10. Hypertension in pregnancy. Amsterdam: Elsevier Science Publisher B.V., 1988:257-266.

46. Halbrecht IG, Komlos L. Mixed wife-husband leukocyte cultures in disturbed and pathological pregnancies. Further studies. Isr J Med Sci 1974; 10:1100-1105.

47. Gille J, Williams JH, Hoffman CP. The feto-maternal lymphocyte interaction in preeclampsia and in uncomplicated pregnancy. Eur J Obstet Gynecol Reprod Biol 1977; 7:227-238.

48. Jenkins DM, Need JA, Scott JS, Morris H, Pepper M. Human leucocyte antigens and mixed lymphocyte reaction in severe pre-eclampsia. Br Med J 1978; 1:542-544.

49. Sargent IL, Redman CW, Stirrat GM. Maternal cell-mediated immunity in normal and pre-eclamptic pregnancy. Clin Exp Immunol 1982; 50:601-609. 
50. Ellis SA, Sargent IL, Redman CW, McMichael AJ. Evidence for a novel HLA antigen found on human extravillous trophoblast and a choriocarcinoma cell line. Immunology 1986; 59:595-601.

51. Schmidt CM, Orr HT. Maternal/fetal interactions: the role of the MHC class I molecule HLA-G. Crit Rev Immunol 1993; 13:207-224.

52. Hunt JS, Orr HT. HLA and maternal-fetal recognition. FASEB J 1992; 6:2344-2348.

53. Sibai BM. Immunologic aspects of preeclampsia. Clin Obstet Gynecol 1991; 34:27-34.

54. Chesley LC, Cosgrove RA, Annitto JE. Pregnancy in the sisters and daughters of eclamptic women. Path Microbiol 1961; 24:662-666.

55. Chesley LC, Annitto JE, Cosgrove RA. The familial factor in toxemia of pregnancy. Obstet Gynecol 1968; 32:303-311.

56. Sutherland A, Cooper DW, Howie PW, Liston WA, MacGillivray I. The incidence of severe pre-eclampsia amongst mothers and mothers-in-law of pre-eclamptics and controls. Br J Obstet Gynaecol 1981; 88:785-791.

57. Chesley LC, Cooper DW. Genetics of hypertension in pregnancy: possible single gene control of pre-eclampsia and eclampsia in the descendants of eclamptic women. $\mathrm{Br} \mathrm{J}$ Obstet Gynaecol 1986; 93:898-908.

58. Arngrimsson R, Bjornsson S, Geirsson RT, Bjornsson H, Walker JJ, Snaedal G. Genetic and familial predisposition to eclampsia and pre-eclampsia in a defined population. Br J Obstet Gynaecol 1990; 97:762-769.

59. Cooper DW, Hill JA, Chesley LC, Bryans CI. Genetic control of susceptibility to eclampsia and miscarriage. Br J Obstet Gynaecol 1988; 95:644-653.

60. Liston WA, Kilpatrick DC. Is genetic susceptibility to pre-eclampsia conferred by homozygosity for the same single recessive gene in mother and fetus? $\mathrm{Br} J$ Obstet Gynaecol 1991; 98:1079-1086.

61. Thornton JG, Onwude JL. Pre-eclampsia: discordance among identical twins. BMJ 1991; 303:1241-1242.

62. Hayward C, Livingstone J, Holloway S, Liston WA, Brock DJ. An exclusion map for pre-eclampsia: assuming autosomal recessive inheritance. Am J Hum Genet 1992; 50:749-757.

63. ACOG technical bulletin. Hypertension in Pregnancy. Number 219, January 1996. Int J Gynaecol Obstet 1996; 53:175-183.

64. Dekker GA, de Vries JI, Doelitzsch PM, et al. Underlying disorders associated with severe early-onset preeclampsia. Am J Obstet Gynecol 1995; 173:1042-1048.

65. van Beek E, Ekhart T, de Leeuw PW, Peeters LLH. Preeclampsia (PE) is followed by subclinical abnormalities in volume homeostasis and renal hemodynamics. Am J Obstet Gynecol 1997; 176:S100.

66. Rodgers GM, Taylor RN, Roberts JM. Preeclampsia is associated with a serum factor cytotoxic to human endothelial cells. Am J Obstet Gynecol 1988; 159:908-914. 
67. Tsukimori K, Maeda H, Shingu M, Koyanagi T, Nobunaga M, Nakano H. The possible role of endothelial cells in hypertensive disorders during pregnancy. Obstet Gynecol 1992; 80:229-233.

68. Tsukimori K, Maeda H, Shingu M, Koyanagi T, Nobunaga M, Nakano H. Possible mechanism of vascular damage in pre-eclampsia. J Hum Hypertens 1994; 8:177-180.

69. Smarason AK, Sargent IL, Redman CW. Endothelial cell proliferation is suppressed by plasma but not serum from women with preeclampsia. Am J Obstet Gynecol 1996; 174:787-793.

70. Walker JJ. Hypertension in pregnancy, report of a RCOG meeting: 27 January, 1994. Br J Obstet Gynecol 1994; 101:639-644.

71. Dekker GA, van Geijn HP. Endothelial dysfunction in preeclampsia. Part I: Primary prevention. Therapeutic perspectives. J Perinat Med 1996; 24:99-117.

72. Greer IA, Leask R, Hodson BA, Dawes J, Kilpatrick DC, Liston WA. Endothelin, elastase, and endothelial dysfunction in pre-eclampsia. Lancet 1991; 337:558.

73. Lorentzen B, Endresen MJ, Hovig T, Haug E, Henriksen T. Sera from preeclamptic women increase the content of triglycerides and reduce the release of prostacyclin in cultured endothelial cells. Thromb Res 1991; 63:363-372.

74. Endresen MJ, Lorentzen B, Henriksen T. Increased lipolytic activity and high ratio of free fatty acids to albumin in sera from women with preeclampsia leads to triglyceride accumulation in cultured endothelial cells. Am J Obstet Gynecol 1992; 167:440-447.

75. Smarason AK, Sargent IL, Starkey PM, Redman CW. The effect of placental syncytiotrophoblast microvillous membranes from normal and pre-eclamptic women on the growth of endothelial cells in vitro. Br J Obstet Gynaecol 1993; 100:943-949.

76. Chua S, Wilkins T, Sargent IL, Redman CWG. Trophoblast deportation in preeclamptic pregnancy. Br J Obstet Gynaecol 1991; 98:973-979.

77. Schrier RW. Pathogenesis of sodium and water retention in high-output and lowoutput cardiac failure, nephrotic syndrome, cirrhosis, and pregnancy (1). N Engl J Med 1988; 319:1065-1072.

78. Omar HA, Ramirez R, Gibson M. Properties of a progesterone-induced relaxation in human placental arteries and veins. J Clin Endocrinol Metab 1995; 80:370-373.

79. Jiang CW, Sarrel PM, Lindsay DC, Poole Wilson PA, Collins P. Progesterone induces endothelium-independent relaxation of rabbit coronary artery in vitro. Eur J Pharmacol 1992; 211:163-167.

80. Jiang CW, Sarrel PM, Lindsay DC, Poole-Wilson PA, Collins P. Endotheliumindependent rabbit coronary artery relaxation by 17 beta-oestradiol in vitro. $\mathrm{Br} \mathrm{J}$ Pharmacol 1991; 104:1033.

81. Collins JA. Diagnostic assessment of the ovulatory process. Seminars in Reproductive Endocrinology 1990; 8, Number 3:145-155.

82. Hoeks APG. Non-invasive study of the local mechanical arterial characteristics in humans. In: Safar ME, O'Rourke MF, eds. The arterial system in hypertension. The Netherlands: Kluwer academic publishers, 1993:119-134. 
83. Hoeks AP, Arts TG, Brands PJ, Reneman RS. Comparison of the performance of the RF cross correlation and Doppler autocorrelation technique to estimate the mean velocity of simulated ultrasound signals. Ultrasound Med Biol 1993; 19:727-740.

84. Cole BR, Giangiacomo J, Ingelfinger JR, Robson AM. Measurement of renal function without urine collection. A critical evaluation of the constant-infusion technique for determination of inulin and para-aminohippurate. N Engl J Med 1972; 287:1109-1114.

85. Carpenter HM, Mudge GH. Uptake and acetylation of p-aminohippurate by slices of mouse kidney cortex. J Pharmacol Exp Ther 1980; 213:350-354.

86. Zittan L. Enzymatic hydrolysis of inulin. An alternative way to fructose production. Storch/Stärke 1981; 33(11):5373-5377.

87. Maher FT, Strong CG, Elveback LR. Renal extraction ratios and plasma-binding studies of radioiodinated o-iodohippurate and iodopyracet and of $\mathrm{p}$-aminohippurate in man. Mayo Clin Proc 1971; 46:189-192.

88. Houben AJHM. Early (micro)circulatory haemodynamic changes in type I diabetes mellitus. Thesis, University of Maastricht, Maastricht, The Netherlands. University Press Maastricht, 1993.

89. Cooper KE, Edholm OG, Mottram RF. The blood flow in skin and muscle of the human forearm. J Physiol 1955; 128:258-267.

90. Fawer R, Dettling A, Weihs D, Welti H, Schelling JL. Effect of the menstrual cycle, oral contraception and pregnancy on forearm blood flow, venous distensibility and clotting factors. Eur J Clin Pharmacol 1978; 13:251-257.

91. Walters WA, Shields L. Venous distensibility during the menstrual cycle. Br J Obstet Gynaecol 1977; 84:697-700.

92. Lehmann ED, Gosling RG, Parker JR, de Silva T, Taylor MG. A blood pressure independent index of aortic distensibility. Br J Radiol 1993; 66:126-131.

93. Lehtovirta P. Forearm blood flow in the normal menstrual cycle. Int J Gynaecol Obstet 1982; 20:223-226.

94. Gosling RG, Beasley MG. Effect of oral contraceptives on resting blood flow in the leg. Angiology 1977; 28:588-598.

95. Wong WH, Freedman RI, Levan NE, Hyman C, Quilligan EJ. Changes in the capillary filtration coefficient of cutaneous vessels in women with premenstrual tension. Am J Obstet Gynecol 1972; 114:950-953.

96. Scott AR, Macdonald IA, Bowman CA, Jeffcoate WJ. Effect of phase of menstrual cycle on insulin sensitivity, peripheral blood flow and cardiovascular responses to hyperinsulinaemia in young women with type 1 diabetes. Diabet Med 1990; 7:57-62.

97. Bircher A, de Boer EM, Agner T, Wahlberg JE, Serup J. Guidelines for measurement of cutaneous blood flow by laser Doppler flowmetry. A report from the Standardization Group of the European Society of Contact Dermatitis. Contact Dermatitis 1994; 30:65-72. 
98. Tooke JE, Tindall H, McNicol GP. The influence of a combined oral contraceptive pill and menstrual cycle phase on digital microvascular haemodynamics. Clin Sci 1981; 61:91-95.

99. Harvell J, Hussona Saeed I, Maibach HI. Changes in transepidermal water loss and cutaneous blood flow during the menstrual cycle. Contact Dermatitis 1992; 27:294-301.

100. Hassan AA, Carter G, Tooke JE. Postural vasoconstriction in women during the normal menstrual cycle. Clin Sci 1990; 78:39-47.

101. Frascarolo P, Schutz Y, Jequier E. Decreased thermal conductance during the luteal phase of the menstrual cycle in women. J Appl Physiol 1990; 69:2029-2033.

102. Bartelink ML, Wollersheim H, Theeuwes A, van Duren D, Thien T. Changes in skin blood flow during the menstrual cycle: the influence of the menstrual cycle on the peripheral circulation in healthy female volunteers. Clin Sci 1990; 78:527-532.

103. Paaby P, Brochner Mortensen J, Fjeldborg P, Raffn K, Larsen CE, Moller Petersen J. Endogenous overnight creatinine clearance compared with 51Cr-EDTA clearance during the menstrual cycle. Acta Med Scand 1987; 222:281-284.

104. Nafziger AN, Schwartzman MS, Bertino JSJ. Absence of tobramycin pharmacokinetic and creatinine clearance variation during the menstrual cycle: implied absence of variation in glomerular filtration rate. J Clin Pharmacol 1989; 29:757-763.

105. Girdler SS, Pedersen CA, Stern RA, Light KC. Menstrual cycle and premenstrual syndrome: modifiers of cardiovascular reactivity in women. Health Psychol 1993; 12:180-192.

106. Kool MJ, Van Merode T, Reneman RS, Hoeks AP, Struyker Boudier HA, Van Bortel LM. Evaluation of reproducibility of a vessel wall movement detector system for assessment of large artery properties. Cardiovasc Res 1994; 28:610-614.

107. Chesley LC. Plasma and red cell volumes during pregnancy. Am J Obstet Gynecol 1972; 112:440-450.

108. Schrier RW, Briner VA. Peripheral arterial vasodilation hypothesis of sodium and water retention in pregnancy: implications for pathogenesis of preeclampsiaeclampsia. Obstet Gynecol 1991; 77:632-639.

109. Chapman AB, Zamudio S, Woodmansee W, et al. Systemic and renal hemodynamic changes in the luteal phase of the menstrual cycle mimic early pregnancy. Am J Physiol 1997; 273:F777-F782.

110. Duvekot JJ, Cheriex EC, Tan WD, Heidendal GA, Peeters LL. Volume-dependent echocardiographic parameters are not useful for estimating baseline blood volume but are useful for detecting acute changes in vascular filling state. Basic Res Cardiol 1994; 89:270-277.

111. van Kreel BK. An improved bromide assay for the estimation of extracellular water volume by capillary gas chromatography. Clin Chim Acta 1994; 231:117-128.

112. Duvekot JJ. Maternal hemodynamic and volume adaptation to pregnancy: an observational study in normal pregnancies and pregnancies complicated by fetal growth re- 
tardation. Thesis, University of Maastricht, Maastricht, The Netherlands. University Press Maastricht, 1994.

113. Simon D, Hartmann BJ, Badouaille G, et al. Two-site direct immunoassay specific for active renin. Clin Chem 1991; 38:1959-1962.

114. Nussberger J, Brunner DB, Waeber B, Brunner HR. True versus immunoreactive angiotensin II in human plasma. Hypertension 1985; 7 (Suppl I): I1-I7.

115. Kubasik NP, Warren K, Sine HE. Evaluation of a new commercial radioassay kit for aldosterone using a iodinated tracer. Clin Biochem 1978; 12:59-61.

116. Rosmalen FM, Tan AC, Tan HS, Benraad TJ. A sensitive radio-immunoassay of atrial natriuretic peptide in human plasma, using a tracer with an immobilized glycouril reagent. Clin Chem Acta 1987; 165:331-340.

117. van der Hoorn FA, Boomsma F, Man in 't Veld AJ, Schalekamp MADH. Determination of catecholamines in human plasma by high-performance liquid chromatography: comparison between a new method with fluorescence detection and an established method with electrochemical detection. J Chromatography 1989; 487:17-28.

118. Huntsman LL, Stewart DK, Barnes SR, Franklin SB, Colocousis JS, Hessel EA. Noninvasive Doppler determination of cardiac output in man. Clinical validation. Circulation 1983; 67:593-602.

119. Bouchard A, Blumlein S, Schiller NB, et al. Measurement of left ventricular stroke volume using continuous wave Doppler echocardiography of the ascending aorta and M-mode echocardiography of the aortic valve. J Am Coll Cardiol 1987; 9:75-83.

120. Kusumoto F, Venet Th, Schiller NB, Sebastian A, Foster E. Measurement of aortic blood flow by Doppler echocardiography: temporal, technician, and reader variability in normal subjects and the application of generalizability theory in clinical research. J Am Soc Echocardiogr 1995; 8:647-653.

121. Lehtovirta P. Haemodynamics of the normal menstrual cycle. Ann Chir Gynaecol Fenn 1974; 63:175-179.

122. Oparil S, Ehrlich EN, Lindheimer MD. Effect of progesterone on renal sodium handling in man: relation to aldosterone excretion and plasma renin activity. Clinical Science and Molecular Medicine 1975; 49:139-147.

123. Davison JM, Noble MCB. Serial changes in 24 hour creatinine clearance during normal menstrual cycles and the first trimester of pregnancy. Br J Obstet Gynecol 1981; $88: 10-17$.

124. Littler WA, Bojorges Bueno R, Banks J. Cardiovascular dynamics in women during the menstrual cycle and oral contraceptive therapy. Thorax 1974; 29:567-570.

125. van Beek E, Houben AJHM, Ekhart THA, et al. Body fluid volume changes during the menstrual cycle (submitted, chapter 4).

126. La Sala GB, Gaddi O, Bruno G, et al. Noninvasive evaluation of cardiovascular hemodynamics during multiple follicular stimulation, late luteal phase and early pregnancy. Fertil Steril 1989; 51:796-802. 
127. van Beek E, Houben AJHM, Ekhart THA, Schiffers PMH, de Leeuw PW, Peeters LLH. Vascular resistance in relation to the menstrual cycle (submitted, chapter 5).

128. Deurenberg P, Weststrate JA, Seidell JC. Body mass index as a measure of body fatness: age- and sex-specific prediction formulas. Br J Nutr 1991; 65:105-114.

129. Kalenga MK, de Gasparo M, Thomas K, De Hertogh R. Down-regulation of angiotensin AT1 receptor by progesterone in human placenta. J Clin Endocrinol Metab 1996; 81:998-1002.

130. Morris NH, Eaton BM, Dekker G. Nitric oxide, the endothelium, pregnancy and pre-eclampsia. Br J Obstet Gynaecol 1996; 103:4-15.

131. Sibai BM. Diagnosis and management of chronic hypertension in pregnancy. Obstet Gynecol 1991; 78:451-461.

132. Rosenn B, Miodovnik M, Combs CA, Khoury J, Siddiqi TA. Poor glycemic control and antepartum obstetric complications in women with insulin-dependent diabetes. Int J Gynaecol Obstet 1993; 43:21-28.

133. van Hooft IM, Grobbee DE, Derkx FH, de Leeuw PW, Schalekamp MA, Hofman A. Renal hemodynamics and the renin-angiotensin-aldosterone system in normotensive subjects with hypertensive and normotensive parents. N Engl J Med 1991; 324:1305-1311.

134. Fisher KA, Luger A, Spargo BH, Lindheimer MD. Hypertension in pregnancy: clinical-pathological correlations and remote prognosis. Medicine Baltimore 1981; 60:267-276.

135. Recommended methods for measurement of red-cell and plasma volume: International Committee for Standardization in Haematology. J Nucl Med 1980; 21: 793- 800.

136. Nisell H, Lintu H, Lunell NO, Mollerstrom G, Pettersson E. Blood pressure and renal function seven years after pregnancy complicated by hypertension. Br J Obstet Gynaecol 1995; 102:876-881.

137. Duvekot JJ, Cheriex EC, Pieters FA, Menheere PP, Peeters LLH. Early pregnancy changes in hemodynamics and volume homeostasis are consecutive adjustments triggered by a primary fall in systemic vascular tone. Am J Obstet Gynecol 1993; 169:1382-1392.

138. Schobel HP, Fischer T, Heuszer K, Geiger H, Schmieder RE. Preeclampsia, a state of sympathetic overactivity. N Engl J Med 1996; 335:1480-1485.

139. Chapman AB, Zamudio S, Woodmansee W, et al. Systemic and renal hemodynamic changes in the luteal phase of the menstrual cycle mimic early pregnancy. Am J Physiol 1997; 273:F777-F782.

140. van Beek E, Peeters LLH. Pathogenesis of preeclampsia: a comprehensive model. Obstet Gynecol Surv 1998; 53:233-239.

141. van Beek E, Ekhart THA, Schiffers PMH, van Eyck J, Peeters LLH, de Leeuw PW. Persistent abnormalities in plasma volume and renal hemodynamics in patients with a history of preeclampsia. Am J Obstet Gynecol 1998; 179:690-696. 
142. Perry IJ, Beevers DG. The definition of pre-eclampsia. Br J Obstet Gynaecol 1994; 101:587-591.

143. Harris EN. Special report. The Second International Anti-cardiolipin Standardization Workshop/the Kingston Anti-Phospholipid Antibody Study (KAPS) group. Am J Clin Pathol 1990; 94:476-484.

144. Derksen RH, Hasselaar P, Blokzijl L, Gmelig Meyling FH, De Groot PG. Coagulation screen is more specific than the anticardiolipin antibody ELISA in defining a thrombotic subset of lupus patients. Ann Rheum Dis 1988; 47:364-371.

145. Vinazzer H, Pangraz U. Protein C: comparison of different assays in normal and abnormal plasma samples. Thromb Res 1987; 46:1-8.

146. Woodhams BJ. The simultaneous measurement of total and free protein $S$ by ELISA. Thromb Res 1988; 50:213-220.

147. Nicolaes GA, Thomassen MC, van Oerle R, et al. A prothrombinase-based assay for detection of resistance to activated protein C. Thromb Haemost 1996; 76:404-410.

148. van Oerle R, van Pampus L, Tans G, Rosing J, Hamulyak K. The clinical application of a new specific functional assay to detect the factor $\mathrm{V}$ (Leiden) mutation associated with activated protein C resistance. Am J Clin Pathol 1997; 107:521-526.

149. Tollefsen DM. Laboratory diagnosis of antithrombin and heparin cofactor II deficiency. Semin Thromb Hemost 1990; 16:162-168.

150. Ubbink JB, Hayward Vermaak WJ, Bissbort S. Rapid high-performance liquid chromatographic assay for total homocysteine levels in human serum. J Chromatogr 1991; 565:441-446.

151. Brun C. A rapid method for determination of para-aminohippuric acid in kidney function test. J Lab Clin Chem 1951; 37:955-958.

152. Roberts JM. Endothelial dysfunction in preeclampsia. Semin Reprod Endocrinol 1998; 16:5-15.

153. Ness RB, Roberts JM. Heterogeneous causes constituting the single syndrome of preeclampsia: A hypothesis and its implications. Am J Obstet Gynecol 1996; 175:1365-1370.

154. Rostrup M, Mundal HH, Westheim A, Eide I. Awareness of high blood pressure increases arterial plasma catecholamines, platelet noradrenaline and adrenergic responses to mental stress. J Hypertens 1991; 9:159-166.

155. Hoyer J. Endothelial vasoregulation and mechanosensitive ion channels in hypertension [editorial]. Nephrol Dial Transplant 1997; 12:6-8.

156. Lebel M, Grose JH, Blais R. Increased hematocrit with normal red blood cell mass in early borderline essential hypertension. Clin Exp Hypertens A 1989; 11:1505-1514.

157. Lim KH, Zhou Y, Janatpour M, et al. Human cytotrophoblast differentiation/invasion is abnormal in pre- eclampsia. Am J Pathol 1997; 151:1809-1818.

158. Guyton AC. Textbook of medical physiology. 6th ed. Philadelphia: W.B. Saunders Company, 1981. 
159. Schalekamp MA, Krauss XH, Schalekamp-Kuyken MP, Kolsters G, Birkenhager WH. Studies on the mechanism of hypernatriuresis in essential hypertension in relation to measurements of plasma renin concentration, body fluid compartments and renal function. Clin Sci 1971; 41:219-231.

160. Sibai BM, Mercer B, Sarinoglu C. Severe preeclampsia in the second trimester: recurrence risk and long-term prognosis. Am J Obstet Gynecol 1991; 165:1408-1412. 



\section{CHAPTER}

Generalized vascular relaxation in the early first trimester can be seen as an important physiologic hallmark of an uncomplicated pregnancy. In contrast, pregnancies complicated by preeclampsia and/or fetal growth restriction are preceded by abnormal hemodynamic adaptation in early pregnancy. The objective of this thesis was to investigate whether in preeclamptic pregnancy maladaptation in central and renal hemodynamics in early pregnancy is mirrored by abnormal hemodynamic changes during the menstrual cycle. In the first part we investigated whether the menstrual cycle mimics the hemodynamic changes of early pregnancy. The second part dealt with the question whether the cyclic hemodynamic changes in women with a history of preeclampsia are different from those observed in women with a history of uneventful pregnancies.

Chapter 2 is a review of the literature in regard to the pathogenesis of preeclampsia. Placental ischemia secondary to defective placentation, a prerequisite for the development of preeclampsia, has a multifactorial origin consisting of three major components: immune maladaptation, genetic predisposition and vascular mediated factors. Probably, a summation of these factors will determine whether or not a pregnant woman is to develop the syndrome.

Chapter 3 describes a pilot-study in which we investigated whether the luteal phase of the menstrual cycle differs from the follicular phase by the development of a state of general vascular relaxation. The results did not support the concept of a luteal state of general vascular relaxation. We could not demonstrate a statistically significant increase in blood flow to the investigated vascular beds (skin, forearm and kidneys) during the luteal phase of the menstrual cycle.

The study in Chapter 4 was designed to investigate whether total peripheral vascular resistance decreases and plasma volume and extracellular fluid volume increase in the luteal phase of the menstrual cycle relative to the follicular phase. Despite the activation of sodium and fluid retaining mechanisms, extracellular fluid volume and plasma volume did not increase appreciably in the luteal phase of the menstrual cycle. Apparently, the stimulus of arterial relaxation, and subsequent arterial underfill, in the luteal phase of the menstrual cycle is, compared to early pregnancy, too weak to elicit a measurable rise in extracellular fluid volume and plasma volume. 
In Chapter 5 we evaluated total peripheral resistance, renal vascular resistance, glomerular filtration rate and forearm vascular resistance in the menstrual cycle during low- and high-salt diet. The data showed, in case of sufficient salt intake, a decrease in both total peripheral resistance and renal vascular resistance without concomitant consistent changes in forearm vascular resistance in the luteal phase of the menstrual cycle. The increase in glomerular filtration rate in the luteal phase is paralleled by an increase in effective renal plasma flow and a decrease in filtration fraction. Post-ovulatory vasodilation of efferent, more than afferent, renal arterioles could be responsible for the raised glomerular filtration rate. The hemodynamic changes induced by the endocrine environment of the luteal phase of the menstrual cycle were modified by salt intake, and mimic the hemodynamic adaptation to early pregnancy.

In Chapter 6 we investigated the relative contribution of endogenous progesterone and $17 \beta$-estradiol to the above described hemodynamic changes during the menstrual cycle by comparing ovulatory - with anovulatory cycles. Except for a difference in cyclic change in serum progesterone, effective renal plasma flow, filtration fraction and renal fraction, we did not find a statistically significant difference in cyclic hemodynamic changes between ovulatory and anovulatory menstrual cycles. Although in many instances both steroids may act in concert in inducing the hemodynamic changes with the menstrual cycle, the results of this study suggest that the luteal fall in total peripheral resistance and rise in glomerular filtration rate are primarily mediated by $17 \beta$-estradiol, whereas, the luteal rise in effective renal plasma flow seems to be primarily a progesterone-dependent effect.

In Chapter 7 we tested the hypothesis that women with a recent history of preeclampsia have abnormalities in their renal hemodynamics and volume status. We observed that women with previous preeclampsia are relatively hypovolemic and tend to have a lower effective renal plasma flow and a higher renal vascular resistance and filtration fraction as compared to controls. These findings support the hypothesis that "healthy" women with a history of preeclampsia show abnormalities in their volume status and renal hemodynamics, irrespective of their blood pressure.

In Chapter 8 we compared cyclic change in volume status and renal hemodynamics in women with a recent history of preeclampsia with that in controls. There were no differences observed in cyclic change between ex-preeclamptics and controls. We only observed differences in baseline values in the subgroups of expreeclamptics with hypertension and apparently healthy ex-preeclamptics as specified in Chapter 7. 
In conclusion, the data presented in this thesis show that hemodynamic changes in the LP of the menstrual cycle resemble those in early pregnancy, and also that these adaptations are dependent upon salt intake. If salt intake is sufficient, a modest general vascular relaxation is observed in the LP of the menstrual cycle. The associated lower luteal TPR seems to be primarily mediated by $17 \beta$-estradiol. Although, sodium and fluid retaining mechanisms are activated, PV and ECFV do not increase appreciably in the LP. The luteal increase in ERPF exceeds that in GFR thus giving rise to a lower FF. The results of our study in anovulatory cycles provides indirect evidence for the concept that the higher ERPF and lower FF in the LP of the menstrual cycle are primarily progesterone-dependent and secondary to selective relaxation of the efferent renal arterioles.

The second part of this thesis demonstrates that, based on our findings of a reduced PV and suppressed level of ANP, women with a history of preeclampsia are relatively hypovolemic. Possibly, a decreased circulatory volume may have caused the observed lower renal blood flow and higher FF. Hemodynamic adaptation during the menstrual cycle in ex-preeclamptics is comparable to that observed in women with a history of uneventful pregnancies. To what extent the hypovolemic state in ex-preeclamptics contributes to the pathogenesis of preeclampsia is the objective of future studies. 


\section{Samenvatting}

Een belangrijk fysiologisch verschijnsel van een ongestoord verlopende zwangerschap is het optreden van een gegeneraliseerde vasodilatatie vroeg in het eerste trimester. In zwangerschappen, die gecompliceerd raken door preëclampsie en/of foetale groeiretardatie, verlopen deze vroege aanpassingen in de bloedsomloop echter afwijkend. Het doel van dit proefschrift was om te onderzoeken of deze vroeg optredende maladaptatie in een door preëclampsie gecompliceerde zwangerschap weerspiegeld wordt door afwijkende hemodynamische veranderingen tijdens de menstruele cyclus. In het eerste gedeelte van dit proefschrift hebben wij onderzocht of de hemodynamische veranderingen van de vroege zwangerschap worden nagebootst in de menstruele cyclus. Het tweede gedeelte behandelt de vraag of hemodynamische veranderingen in de menstruele cyclus bij vrouwen met een preëclampsie in de anamnese vergelijkbaar zijn met die bij vrouwen met een blanco anamnese.

Hoofdstuk 2 behandelt de literatuur met betrekking tot de pathogenese van preëclampsie. Een gestoorde placentatie met als gevolg placentaire ischemie lijkt een voorwaarde voor het ontstaan van preëclampsie. Deze gestoorde placentatie is vaak multicausaal, waarbij er drie categorieën van afwijkingen te onderscheiden zijn, te weten: een afwijkende aanpassing van het afweersysteem, genetische aanleg en vasculair gemediëerde factoren. Waarschijnlijk bepaalt een optelsom van deze factoren of een zwangere vrouw preëclampsie zal ontwikkelen.

Hoofdstuk 3 beschrijft een pilot-studie bij gezonde vrouwen waarbij we de hypothese getoetst hebben, dat er in de luteale fase van de menstruele cyclus in vergelijking tot de folliculaire fase een gegeneraliseerde vasodilatatie optreedt. We zagen in de luteale fase van de cyclus geen significante toename in de doorbloeding van de huid, skeletspier (onderarm) en de nieren. Deze onderzoeksresultaten boden geen ondersteuning voor de geponeerde hypothese. Daarbij dient wel de kanttekening geplaatst te worden, dat de power van de pilot-studie laag was.

De studie in hoofdstuk 4 was ontwikkeld om te onderzoeken of, in vergelijking tot de folliculaire fase, de totale perifere vaatweerstand zou afnemen en het plasmavolume en extra-cellulaire volume zou toenemen in de luteale fase van de menstruele cyclus bij gezonde vrouwen. Ondanks de activatie van zout- en vochtretinerende mechanismen nam het extra-cellulaire volume en plasmavolume niet toe in de luteale fase van de menstruele cyclus. Blijkbaar was de stimulus van arteriële relaxatie, met als gevolg arteriële ondervulling in de luteale fase van de 
menstruele cyclus, te zwak in vergelijking tot die in de zwangerschap, om een meetbare toename te veroorzaken in het extra-cellulaire volume en plasmavolume.

In hoofdstuk 5 hebben wij de totale perifere vaatweerstand, renale vaatweerstand, glomerulaire filtratiesnelheid en vaatweerstand in de onderarm onderzocht tijdens de menstruele cyclus bij gezonde vrouwen die achtereenvolgens een laag - en hoogzout dieet gebruikten. Alleen bij een hoogzout dieet tijdens de luteale fase van de menstruele cyclus konden wij een afname van de totale perifere vaatweerstand en renale vaatweerstand aantonen met een gelijkblijven van de vaatweerstand in de onderarm. De toename van de glomerulaire filtratiesnelheid ging vergezeld van een toename van de effectieve renale plasma doorstroming en een afname in de filtratie fractie. De luteale toename in glomerulaire filtratiesnelheid zou een gevolg kunnen zijn van een sterkere vaatverwijding van de efferente renale arteriolen, die vanuit de glomerulus de peritubulaire microcirculatie verzorgen, dan van de afferente renale arteriolen naar de glomerulus toe. De luteale veranderingen in bloedsomloop bleken afhankelijk te zijn van de zoutconsumptie en tonen overeenstemming met het patroon van hemodynamische veranderingen, die men in de vroege zwangerschap ziet.

In hoofdstuk 6 werd de relatieve bijdrage van endogeen progesteron en $17 \beta$-oestradiol aan de bovengenoemde veranderingen in de bloedsomloop tijdens de menstruele cyclus bestudeerd door ovulatoire met anovulatoire cycli te vergelijken. De cyclische veranderingen in ovulatoire en anovulatoire cycli waren voor bijna alle gemeten variabelen vergelijkbaar, met uitzondering van de mate van toename in het serum progesteron, de effectieve renale plasma doorstroming, de filtratie fractie en de renale fractie van het hartminuutvolume. Hoewel meestal beide steroïden samenwerken bij het veroorzaken van hemodynamische veranderingen tijdens de menstruele cyclus, suggereren deze resultaten dat de luteale afname in totale perifere vaatweerstand en toename in glomerulaire filtratiesnelheid voornamelijk veroorzaakt worden door $17 \beta$-oestradiol. De luteale toename in effectieve renale plasma doorstroming lijkt voornamelijk veroorzaakt te worden door progesteron.

In hoofdstuk 7 hebben wij de hypothese getoetst, dat vrouwen met preëclampsie in de voorgeschiedenis afwijkingen vertonen in renale hemodynamiek en plasmavolume. Wij konden aantonen dat vrouwen met preëclampsie in de voorgeschiedenis in vergelijking tot vrouwen met een blanco voorgeschiedenis een verlaagd plasmavolume hebben, vaak gepaard gaand met een lagere effectieve renale plasma doorstroming enerzijds, en een hogere renale vaatweerstand en filtratie fractie anderzijds. Deze bevindingen ondersteunen de hypothese, dat ogenschijnlijk gezonde vrouwen, die in het verleden een preëclampsie hebben doorgemaakt, een 
verlaagd plasmavolume hebben vaak in combinatie met een licht gecompromitteerde renale hemodynamiek.

In hoofdstuk 8 hebben we cyclische veranderingen in plasmavolume en renale hemodynamiek bestudeerd in een groep vrouwen met preëclampsie in de voorgeschiedenis en deze vergeleken met een groep vrouwen, die een ongestoorde zwangerschap hadden doorgemaakt. Alhoewel de vrouwen zowel een lager plasmavolume hadden alsook een licht verlaagde renale hemodynamiek (zie hoofdstuk 7), waren alle cyclische veranderingen tussen de groepen vergelijkbaar.

Concluderend kunnen we stellen, dat met de data in dit proefschrift wordt aangetoond dat de hemodynamische veranderingen in de luteale fase van de menstruele cyclus lijken op die in de vroege zwangerschap, met als bijzonderheid, dat de amplitudo van de cyclische veranderingen toeneemt naarmate er meer zout geconsumeerd wordt. $\mathrm{Bij}$ voldoende zout inname wordt er een bescheiden gegeneraliseerde vasodilatatie waargenomen in de luteale fase van de menstruele cyclus. De lagere totale perifere vaatweerstand in de luteale fase lijkt voornamelijk veroorzaakt door het $17 \beta$-oestradiol. Alhoewel zout- en vochtretinerende mechanismen zijn geactiveerd, nemen plasmavolume en extra-cellulair volume niet toe in de luteale fase. De luteale toename van de effectieve renale plasma doorstroming is groter dan die van de glomerulaire filtratiesnelheid, waardoor de filtratie fractie daalt. Onze bevindingen in de studie van anovulatoire cycli ondersteunen de idee, dat de hogere effectieve renale plasma doorstroming en lagere filtratie fractie in de luteale fase van de menstruele cyclus voornamelijk effecten van progesteron zijn, hetgeen mogelijk tot stand komt door een selectieve relaxatie van efferente renale arteriolen.

Het tweede deel van dit proefschrift toont aan dat, gebaseerd op onze bevindingen van een verlaagd plasmavolume en ANP, de vullingsgraad van het vaatbed bij vrouwen met preëclampsie in de voorgeschiedenis krapper is. Mogelijk dat een verlaagd circulerend volume de oorzaak is van de waargenomen lagere nierdoorbloeding en hogere filtratie fractie. De hemodynamische veranderingen tijdens de menstruele cyclus bij vrouwen met preëclampsie in de voorgeschiedenis zijn vergelijkbaar met die bij vrouwen met een blanco voorgeschiedenis. In hoeverre de verminderde vullingsgraad bij vrouwen met preëclampsie in de voorgeschiedenis een rol speelt in de pathogenese van preëclampsie is het doel van toekomstige studies. 


\section{Publications}

The validity of screening for small for gestational age and underweight for length infants by Doppler ultrasound. EA Sijmons, PJHM Reuwer, E van Beek, HW Bruinse. Br J Obstet Gynaecol 1989; 96, 557-561.

Umbilical artery velocimetry and non-stress test in monitoring high-risk pregnancies. A comparative longitudinal study. P Malcus, E van Beek, K Maršál. Ultrasound Obstet Gynecol 1991; 1, 95-101.

Peripheral hemodynamics and renal function in relation to the menstrual cycle. E van Beek, AJHM Houben, PN van Es, C Willekes, ECCM Korten, PW de Leeuw, LLH Peeters. Clinical Science 1996; 91, 163-168.

De pathogenese van preëclampsie. E van Beek. LLH Peeters. Ned Tijdschr Geneeskd 1997; 141 (28), 1379-84.

Pathogenesis of preeclampsia: a comprehensive model. E van Beek, LLH Peeters. Obstet Gynecol Survey 1998; 53, 233-239.

Persistent abnormalities in plasma volume and renal hemodynamics in patients with a history of preeclampsia. E van Beek, THA Ekhart, PMH Schiffers, J van Eyck, LLH Peeters, PW de Leeuw. Am J Obstet Gynecol 1998; 179:690-6.

A new method for plasma volume measurements with unlabeled dextran-70 instead of ${ }^{125} \mathrm{I}$-labeled albumin as an indicator. BK van Kreel, E van Beek, MEA Spaanderman, LLH Peeters. Clin Chim Acta 1998 Jul 6; 275(1):71-80. 


\section{Nawoord}

Bij de totstandkoming van dit proefschrift zijn in de loop der jaren veel mensen op enigerlei wijze betrokken geraakt. Zonder hun hulp zou het mij beslist niet gelukt zijn dit proefschrift te schrijven. Ik zou dan ook allen graag willen bedanken middels het promotiefeest.

Toch wil ik enkelen speciaal noemen zonder hiermee anderen te kort te willen doen.

Liefste Margreet, door jouw onvoorwaardelijke steun en kritische blik tijdens deze zeven jaren van opleiding en onderzoek, ook op de momenten dat het wat minder ging, heb ik beide tot een goed einde kunnen brengen. Daarom heb ik dit proefschrift aan jou opgedragen, wetende dat het nooit was verschenen zonder jouw steun. Daarnaast stond jij altijd klaar voor onze kinderen en wist je dit te combineren met het huisartsenvak. Ik heb de grootste bewondering voor jou.

Beste Louis, door jouw creatieve geest en inzet is deze onderzoekslijn en ook dit proefschrift voor een belangrijk deel tot stand gekomen. Alhoewel, of misschien wel juist omdat mijn enigszins calvinistische inslag niet altijd spoorde met jouw meer Zuidelijke kijk op de gang van zaken, wat betreft interpretatie van het onderzoek en de organisatie van de onderzoekslijn, is onze samenwerking heel vruchtbaar geweest.

Beste Peter, door jouw nuchtere wetenschappelijke analyses en grote nefrologische kennis heb je ons menigmaal behoed voor al te wilde speculaties. Veel dank voor je begeleiding.

Beste professor de Haan, zowel opleider als promotor, ongetwijfeld heeft $u$, naast Hans Brölmann, grote invloed gehad op mijn vorming tot gynaecoloog. Mede uw stimulering om wetenschappelijk onderzoek te doen naast de opleiding tot gynaecoloog is van belang geweest bij het tot stand komen van dit proefschrift. Met name de inpassing van het onderzoeksproject binnen mijn opleidingsschema werd door u gesteund en georganiseerd. 


\section{Curriculum vitae}

Erik van Beek werd op 20 maart 1963 geboren te Putten (Gelderland). In 1981 behaalde hij het atheneum-B diploma aan het Revius Lyceum te Doorn. Van 1981 tot 1982 studeerde hij Scheikunde aan de Rijks Universiteit Utrecht. Aan deze universiteit studeerde hij geneeskunde van 1982 tot en met 1990 (doctoraal examen: 21 november 1986, arts examen: 23 februari 1990). Voor aanvang van de co-schappen liep hij een wetenschappelijke stage aan de medische faculteit (vakgroep gynaecologie) van de Universiteit van Lund in Zweden (begeleider: dr. K. Maršál).Van februari tot oktober 1990 werkte hij als arts-assistent chirurgie in het ziekenhuis de Weezenlanden te Zwolle. Van oktober 1990 tot november 1991 werkte hij als arts-assistent gynaecologie in het AMC te Amsterdam. Na een kortdurende aanstelling als arts-onderzoeker bij de vakgroep obstetrie in het AZL te Leiden (begeleider: prof. dr. H.H. Kanhai) werd op 16 februari 1992 gestart met de opleiding tot gynaecoloog in het AZM te Maastricht (opleider: prof. dr. J. de Haan). Van februari 1995 tot april 1997 was hij werkzaam in het AZM te Maastricht als arts-onderzoeker aan het project, gesubsidieerd door de Nierstichting Nederland, dat de basis vormde voor dit proefschrift. Vanaf april 1997 vervolgde hij zijn opleiding tot gynaecoloog in het Sint Joseph Ziekenhuis te Veldhoven (opleider: dr. H.A.M. Brölmann). Op 16 februari 1999 hoopt hij bijgeschreven te worden in het register van medische specialisten. 
Publication of this thesis was financially supported by

Ferring Nederland BV

Janssen-Cilag BV

Organon Nederland BV

Pie Medical

Schering Nederland BV

Serono Benelux BV

Zeneca Farma 

